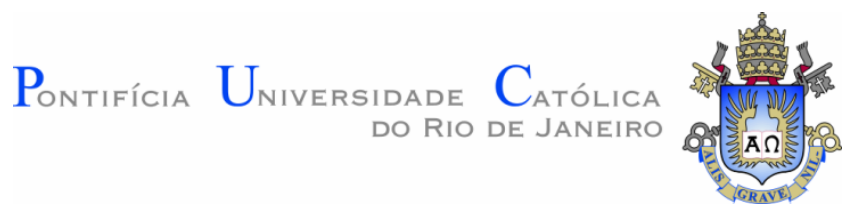

Jorge Virgilio de Almeida

Virtual Magnetic Transmission Lines

Dissertação de Mestrado

Dissertation presented to the Programa de Pósgraduação em Engenharia Elétrica of PUC-Rio in partial fulfillment of the requirements for the degree of Mestre em Engenharia Elétrica.

Advisor: Prof. Gláucio Lima Siqueira Co-advisor: Prof. Marbey Manhães Mosso 
Jorge Virgilio de Almeida

\section{Virtual Magnetic Transmission Lines}

Dissertation presented to the Programa de Pós-graduação em Engenharia Elétrica of PUC-Rio in partial fulfillment of the requirements for the degree of Mestre em Engenharia Elétrica. Approved by the undersigned Examination Committee.

Prof. Gláucio Lima Siqueira

Orientador

Centro de Estudos em Telecomunicações - CETUC

Prof. Marbey Manhães Mosso

Co-orientador

Centro de Estudos em Telecomunicações - CETUC

Prof. Carlos Antonio França Sartori Comissão Nacional de Energia Nuclear em São Paulo

Profa. Maria Cristina Ribeiro Carvalho Centro de Estudos em Telecomunicações - CETUC

Profa. Gelza de Moura Barbosa Marinha do Brasil

Prof. Márcio da Silveira Carvalho

Vice Dean of Graduate Studies Centro Técnico Científico - PUC-Rio 
All rights reserved.

\section{Jorge Virgilio de Almeida}

The author graduated in Electric Engineering from Pontifícia Universidade Católica do Rio de Janeiro (PUC-Rio) in 2015 and received the title of ingénieur diplomé from École Centrale de Lyon in 2016.

Bibliographic data

De Almeida, Jorge Virgilio

Virtual magnetic transmission lines / Jorge Virgilio de Almeida ; advisor: Gláucio Lima Siqueira ; co-advisor: Marbey Manhães Mosso. - 2017.

120 f. : il. color. ; $30 \mathrm{~cm}$

Dissertação (mestrado)-Pontifícia Universidade Católica do Rio de Janeiro, Departamento de Engenharia Elétrica, 2017.

Inclui bibliografia

1. Engenharia Elétrica - Teses. 2. Linhas de transmissão virtuais. 3. Transmissão de energia sem fio. 4. Metamateriais. 5. Propriedades eletromagnéticas negativas. 6. Impedâncias não fosterianas. I. Siqueira, Gláucio Lima. II. Mosso, Marbey Manhães. III. Pontifícia Universidade Católica do Rio de Janeiro. Departamento de Engenharia Elétrica. IV. Título.

CDD: 621.3 


\section{Acknowledgements}

This dissertation is dedicated to my parents, Ivete Lobato de Almeida and Jorge Vitoriano de Almeida.

Said that, l'd like to thank my advisors, Prof. Glaucio Lima Siqueira and Prof. Marbey Manhães Mosso, for supporting me during all my studies. To Prof. Marco Antonio Grivet Mattoso Maia and Prof. Ana Maria Beltran Pavani for being there whenever I needed. To Prof. Carlos Antonio França Sartori, Prof. Laurent Krähenbühl and Prof. Christian Vollaire for also being there even being far away. To Prof. Maria Cristina Ribeiro Carvalho for her cooperation. To Conselho Nacional de Desenvolvimento Científico e Tecnológico (CNPq) and to Pontifícia Universidade Católica do Rio de Janeiro (PUC-Rio) for the scholarship and financial aid granted to me. To all my laboratory colleagues from the Center for Telecommunication Research (CETUC) for their complicity: Renato Feitoza, Gidy Florez, Jorge Mitrione, Ilidio Ferreira, Juliana Carvalho, Vinícius Lima, Gretell Capote, Felipe Calliari, Rodrigo Santos, Marcelo Balisteri, Carolina Maria Amaral and Gelza Barbosa. To the staff of the CETUC and of the Department of Electrical Engineering without whom none of this would be possible. To my friends for life Pedro Furlani, Luiz Bez, Flávio de Assis, Mayara Lobato, Wilson Luciano and Grasiele Maciel for their companionship. And also to my aunt Ivanilde Lobato for her dedication to me.

I'd like to especially thank Julio Nicolini for his important contribution to the realization of this work.

And finally l'd like to thank Maria Freire for everything else. 


\section{Abstract}

De Almeida, Jorge Virgilio; Siqueira, Gláucio Lima (Advisor); Mosso, Marbey Manhães (Co-advisor). Virtual Magnetic Transmission Lines. Rio de Janeiro, 2017. 120p. Dissertação de Mestrado Departamento de Engenharia Elétrica, Pontifícia Universidade Católica do Rio de Janeiro.

Over recent years, the interest in using inductive wireless power transmission for many applications has grown. One of the major limitations of this technology is the reduced operating distance. Some recent works have suggested using artificial materials known as metamaterials to improve the power transfer efficiency over distance. Due to their unique electromagnetic properties, such as negative permeability, metamaterials can be used to enhance the evanescent waves of the near field. In the present work, the usage of an electromagnetic metamaterial to increase the inductive coupling by means of enhanced evanescent waves is studied. Analytical calculations and numerical simulations of the proposed metamaterial are presented. The improvement of the power transfer efficiency is supported by empirical evidences.

\section{Keywords}

Virtual transmission lines; metamaterial; negative permittivity; negative permeability; negative refractive index; non-foster impedance. 


\section{Resumo}

De Almeida, Jorge Virgilio; Siqueira, Gláucio Lima (Orientador); Mosso, Marbey Manhães (Co-orientador). Linhas de transmissão magnéticas virtuais. Rio de Janeiro, 2017. 120p. Dissertação de Mestrado - Departamento de Engenharia Elétrica, Pontifícia Universidade Católica do Rio de Janeiro.

Nos últimos anos, tem aumentado o interesse no uso da transmissão de energia sem fio por acoplamento indutivo em muitas aplicações. Uma das maiores limitações dessa tecnologia é a distância de operação reduzida. Alguns trabalhos recentes sugerem usar materiais artificiais conhecidos como metamateriais para aprimorar a eficiência da transferência de potência ao longo da distância. Devido às suas propriedades eletromagnéticas únicas, tais como permeabilidade magnética negativa, metamateriais podem ser usados para amplificar as ondas evanescentes do campo próximo. No presente trabalho, é estudado o uso de metamateriais eletromagnéticos para aumentar o acoplamento indutivo por meio da amplificação do campo próximo. São apresentados cálculos analíticos e simulações dos metamateriais propostos. O melhoramento da eficiência na transferência de potência é apoiado por evidências experimentais.

\section{Palavras-chave}

Linhas de transmissão virtuais; metamaterial; permissividade negativa; permeabilidade negativa; índice de refração negativo; impedância não fosteriana. 


\section{Contents}

1 Introduction

2 Theoretical framework 19

2.1. Introduction 19

2.2. Definitions 19

2.3. Electromagnetic properties of materials 20

2.3.1. Electric properties of materials 22

2.3.2. Magnetic properties of materials 24

2.4. Physical interpretation of negative-index materials 28

2.5. Classification of electromagnetic materials 32

2.6. Fundamental concepts of electromagnetic field theory 33

2.6.1. Basic field and circuit equations 33

2.6.2. Travelling EM wave $\quad 34$

2.6.3. Power and energy in materials 35

2.7. Velocity of power transportation 38

2.8. Causality of negative-index materials 39

2.9. Wave propagation in material media 39

2.10. Electromagnetic coupling 43

3 Generalized theory of transmission lines 46

3.1. Introduction: transmission line basic concepts 46

3.2. Electro-motive and magneto-motive forces 49

3.3. Physical transmission lines 56

3.3.1. Electric transmission line 56

3.3.2. Magnetic transmission line 57

3.4. Virtual transmission lines $\quad 59$

3.4.1. Magnetic transmission line $\quad 59$

3.4.2. Electric transmission line 62

3.5. Solution of the propagation equations and time-average powers 64

3.6. Free-space virtual magnetic lines 67 
4 Implementation of virtual impedances

69

4.1. Introduction 69

4.2. Metamaterials 72

4.3. Realization of negative-index materials 73

4.3.1. $\varepsilon$-negative metamaterial 74

4.3.2. $\boldsymbol{\mu}$-negative metamaterial $\quad 75$

4.4. Proposed prototype for a $\boldsymbol{\mu}$-negative metamaterial 79

4.4.1. Parameters 79

4.4.2. Retrieval of the effective permeability 83

4.4.3. Equivalent virtual impedance 84

5 Magnetic transmission line assisted by metamaterial 88

5.1. Proposed system 88

5.2. Analytical and numerical results for the proposed system 90

5.2.1. Simulated effective permeability and virtual impedance 91

5.2.2. Comparison between analytical and numerical results for the proposed system without the metamaterial 95

5.2.3. Comparison between analytical and numerical results for the proposed system with the metamaterial

5.3. Simulations of the EM-field distribution for the proposed system

100

5.4. Experimental results for the proposed system 108

5.4.1. Experimental protocol 108

5.4.2. Results 111

6 Conclusion 114

6.1. Main results 114

6.2. Contributions 115

6.3. Future works and perspectives 116

Bibliography 


\section{Table of Figures}

Figure 1.1: Wardenclyffe Tower $\quad 15$

Figure 1.2: Typical non-radiative WPT-based charging systems $\quad 16$

Figure 2.1: (a) Orbital motion and (b) spin of an electron 25

Figure 2.2: Cross section of a magnetized material 26

Figure 2.3: Dielectric function of a plasma. Propagation is only possible above the plasma frequency $\omega p$. EM wave is reflected when $\varepsilon^{\prime}$ is negative [10]

Figure 2.4: Electromagnetic-field zones as a function of the wavelength $\lambda$

Figure 2.5: One of the fields is predominant in the near-field zone

Figure 3.1: The difference of magnetic potential between the incoming and ongoing fluxes causes a transversal magnetic field component to be stored between them. It is this component that is responsible for power flow, not the magnetic field paralell to the flux

Figure 3.2: For guided modes, one of the fields is the source of a real potential. For radiated modes, both fields are circulations (hence, virtual potentials)

Figure 3.3: Schematic of an electric transmission line 56

Figure 3.4: Schematic of a magnetic transmission line 58

Figure 3.5: Terminals of a virtual magnetic transmission line $\quad 59$

Figure 3.6: Schematic of a virtual magnetic transmission line $\quad 60$

Figure 3.7: Schematic of a virtual electric transmission line 62

Figure 4.1: Classification of materials according to the sign of its properties

Figure 4.2: In negative-index media the phase velocity is reversed in relation to the direction of the power flow

Figure 4.3: Perfect lensing implies the focalization of the far field (A) and the near field (B) 
Figure 4.4: MTMs are artificial crystals made of engineered atoms and lattice

Figure 4.5: DNG structure made out of SNG MTMs in different planes 74

Figure 4.6: TL-based cell $\quad 75$

Figure 4.7: (a) Split-ring and (b) spiral resonator based cells $\quad 76$

Figure 4.8: Resonance control with lumped capacitors 77

Figure 4.9: The focalization of the evanescent modes enhances the magnetic coupling 78

Figure 4.10: Proposed unit cell 80

Figure 4.11: Equivalent circuit of a SR-based cell 81

Figure 4.12: Representation of the front side of MTM prototype 82

Figure 4.13: Representation of the back side of MTM prototype 83

Figure 4.14: Estimation of the real (red solid) and imaginary part (blue dash) of $\mu r$

Figure 4.15: Reactance XMTM as a function of frequency 85

Figure 4.16: First frequency derivative of XMTM 86

Figure 4.17: MTM equivalent impedance $Z m, v$ as a function of frequency

Figure 5.1: Simulation of the proposed system on ADS

Figure 5.2: Numerical result for the real (red solid) and imaginary part (blue dash) of $\mu r$ on ADS

Figure 5.3: Numerical result for the real (red solid) and imaginary part (blue dash) of XMTM on ADS

Figure 5.4: Numerical result for the real (red solid) and imaginary part (blue dash) of the first frequency derivative of $X M T M$ on ADS

Figure 5.5: Numerical result for the real (red solid) and imaginary part (blue dash) of ZMTM on ADS

Figure 5.6: Analytical results for the magnitude (solid) and the phase (short dash) of $Z 0$ without the MTM slab

Figure 5.7: Numerical results for the magnitude (solid) and the phase (short dash) of $Z 0$ without the MTM slab 
Figure 5.8: Analytical (solid line) and numerical (circle) results for the magnitude of $\Gamma$ without the MTM slab

Figure 5.9: Analytical (solid line) and numerical (circle) results for the gain $G$ without the MTM slab

Figure 5.10: Analytical results for the magnitude (solid) and the phase (short dash) of $Z 0$ without the MTM slab

Figure 5.11: Numerical results for the magnitude (solid) and the phase (short dash) of $Z 0$ without the MTM slab

Figure 5.12: Analytical (solid line) and numerical (circle) results for the magnitude of $\Gamma$ with the MTM slab

Figure 5.13: Analytical (solid line) and numerical (circle) results for the gain $G$ with the MTM slab

Figure 5.14: a MGTL is analogous to a high-pass filter

Figure 5.15: Simulation of the proposed system on EMPro

Figure 5.16: Magnitude of $\mathrm{Hz}$ at the sub-resonance range at the plane $\mathrm{YZ}$

100

Figure 5.17: Magnitude of $\mathrm{Hz}$ at the over-resonance range at the plane $\mathrm{YZ}$

101

Figure 5.18: Magnitude of $\mathrm{Hz}$ at the resonance at the plane $\mathrm{YZ}$

Figure 5.19: Magnitude of $\mathrm{Hz}$ at the sub-resonance range at the MTM surface

Figure 5.20: Magnitude of $\mathrm{Hz}$ at the over-resonance range at the MTM surface

Figure 5.21: Magnitude of $\mathrm{Hz}$ at the resonance at the MTM surface

Figure 5.22: Focalization of $\mathrm{Hz}$ is deviated from the center due to material losses

Figure 5.23: Magnitude of $H x$ at the sub-resonance range at the plane $\mathrm{XZ}$

Figure 5.24: Magnitude of $H x$ at the over-resonance range at the plane $X Z$

Figure 5.25: Magnitude of $H x$ at the resonance at the plane $X Z$

Figure 5.26: Simulation of the proposed system on CST Studio 
Figure 5.27: The normal component of $\mathrm{Hz}$ at the surface of the MTM out of the resonance

Figure 5.28: The normal component of $\mathrm{Hz}$ at the surface of the MTM at the resonance

Figure 5.29: Inversion of the magnetization curve derivative

Figure 5.30: The tangential components of $\boldsymbol{H}$ at the surface of the MTM are virtually zero inside the unit-cell area at the resonance

Figure 5.31: Schematic of the setup used to measure the Sparameters of the system

Figure 5.32: Experimental setup without the MTM slab 109

Figure 5.33: Experimental setup with the MTM slab

Figure 5.34: Experimental result for the gain $G$ without the MTM slab

Figure 5.35: Experimental result for the gain $G$ with the MTM slab

Figure 5.36: The impedance of the source loop is affected if the MTM is too close

Figure 5.37: The MTM behaves as a capacitor added to the source loop when its separated from it a distance smaller than $a$ 


\section{Acronyms}

ADS Advanced Design System

DNG Double Negative

DPS Double Positive

ELTL Electric Transmission Line

EM Electromagnetic

ENG Epsilon Negative

IWPT Inductive Wireless Power Transmission

LHM Left-Handed Material

MGTL Magnetic Transmission Line

MNG Mu Negative

MSRR Multiple Split Ring Resonator

MTM Metamaterial

U

NRI Negative Refractive Index

PCB Printed Circuit Board

SNG Single Negative

SR Spiral Resonator

SRR Split Ring Resonator

VELTL Virtual Electric Transmission Line

VMGTL Virtual Magnetic Transmission Line

VNA Vector Network Analyzer

WPC Wireless Power Consortium

WPT Wireless Power Transmission 
"Let the future tell the truth, and evaluate each one according to his work and accomplishments. The present is theirs; the future, for which I have really worked, is mine."

Nikola Tesla 


\section{Introduction}

The dream of wireless power transmission (WPT) dates back to Tesla [1]. Almost a century after his first attempts to create a unified global wireless system for telecommunications and power transmission (see Figure 1.1), the subject is reemerging due to the increasing demand on autonomy for electronic devices.

Most of the daily life gadgets (like smartphones, tablets or laptops) and the robots employed in industry (like the Kiva robots at Amazon's warehouses) are cordless. Nonetheless, their autonomy is limited by their dependence on batteries. Battery technology is not only an expensive and environmentally unfriendly technology, it is also highly time-consuming. For that reason, more efficient WPT systems are quite promising in every level: from implantable medical devices to electric vehicles.

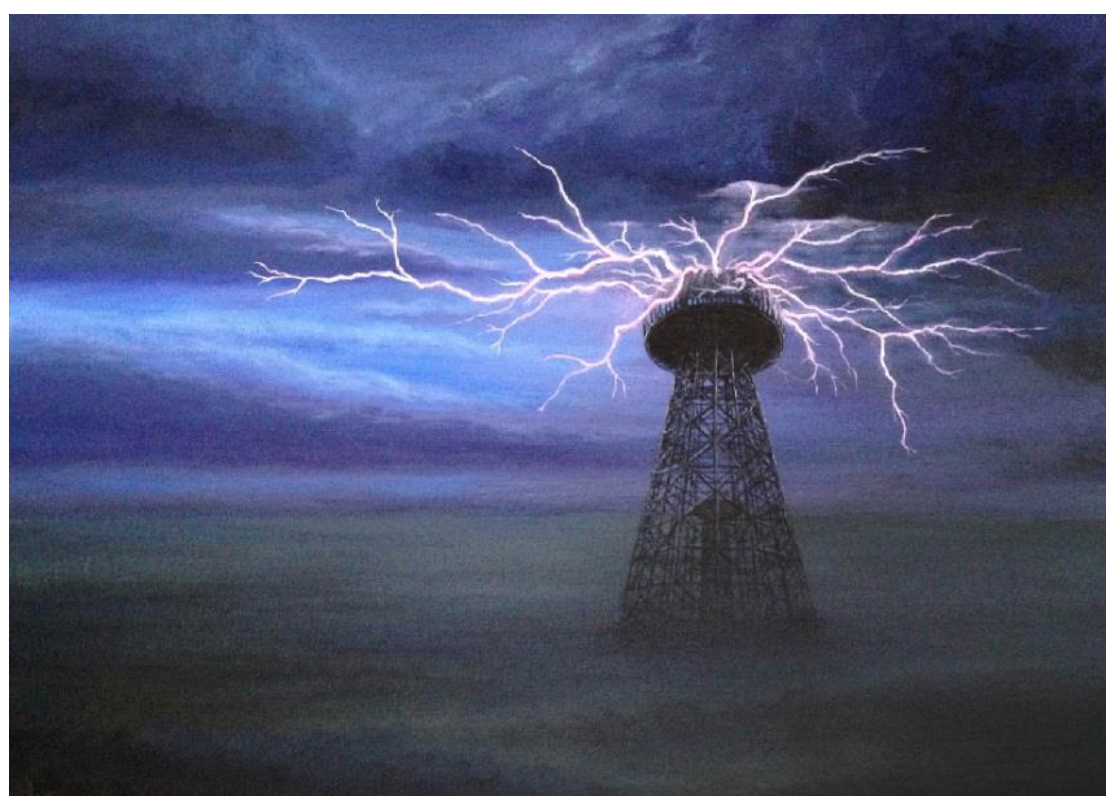

Figure 1.1: Wardenclyffe Tower 


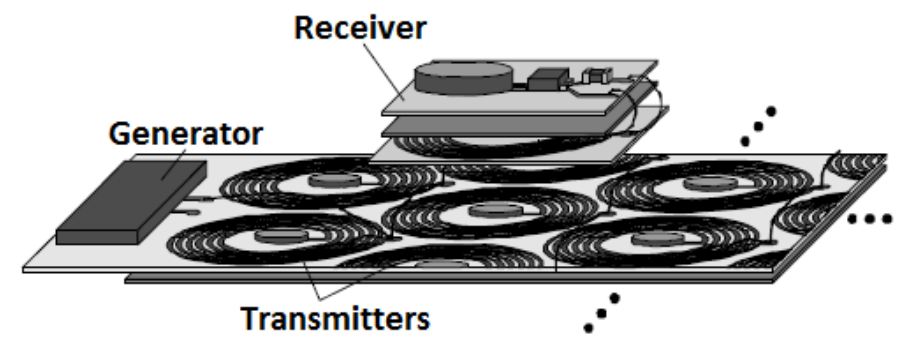

Figure 1.2: Typical non-radiative WPT-based charging systems

According to the Forbes' list [2], the six more prominent companies working on the development of WPT systems are: Powermat, PowerByProxi, Wireless Power Consortium (WPC), Mojo Mobility, WiPower and Mopar.

WPC is an open-membership of more than 150 companies from Asia, Europe and North America, including big mobile manufactures like Samsung, who is working since 2008 toward a global standardization of wireless charging technologies [3]. Since 2009, companies who are members of WPC adopt Qi (the chinese word for "energy") as their lowpower inductive wireless power transmission (IWPT) standard.

Although the electromagnetic (EM) field is much more efficient transmitting power over large distance in its radiative form, the human exposure to electromagnetic waves carrying power on the order of tens of watts is tremendously risk. So, the energy transfer by non-radiative modes is considered much more suitable concerning health and safety. Once the magnetic field is supposed to interact very weakly with biological beings, the majority of the WPT systems exploit the magnetic near field instead of the electric one (see Figure 1.2).

One of the main limitations of IWPT is its short operating distance (on the order of the coil diameter). In 2007, a group of Massachusetts Institute of Technology (MIT) researchers demonstrated that by achieving strong magnetic coupling efficient WPT over midrange (a few times the coil diameter) was possible. This technique, named Witricity by its creators, exploits the high-Q resonance coupling of a 4-coil topology as mean to compensate the decay of the coupling coefficient with the increasing distance between source and load drivers. It can be demonstrated that 
power transfer efficiency (PTE) can be made arbitrarily close to $100 \%$ by increasing the Q-factor of the receiver and transmitter coils, which also makes those systems quite robust to misalignment.

Besides Witricity virtues, it is too susceptible to splitting frequency, especially in a multi-receiver scenario, which imposes a great limitation to the power delivered to the load (PDL). Another problem considering this technique is the energy waste due to leakage flux of a non-directional magnetic field. If the magnetic field could be made more directional, PTE could be highly increased without the need of High-Q resonance coupling between the coils. Some solutions, like strategically designing the coils or, more elegantly, shaping the magnetic field by phase controlling the current loops, have been proposed [4].

However, recent works indicate that artificial complex media called metamaterials (MTM) are potential key technology for efficient IWPT systems [5] [6]. Being structures whose electromagnetic parameters can be fully engineered, MTMs are able to synthesized effective negative materials that permit to focus the magnetic near field and to enhance the magnetic coupling between the drivers [7].

In order to better understand this enhancement phenomenon of the magnetic coupling caused by the presence of a MTM in the inductive link and to stress its underlying mechanisms, the present work proposes to study the power transfer between two inductively coupled coils based on transmission line theory.

As it is shown in the next chapters, coupled circuits behave as virtual transmission lines and by interpreting the MTM slabs as virtual impedances connected to this line, the MTM-enhanced coupling appears as a consequence of the improvement of the power factor of the line.

Chapter 2 presents a brief review of the fundamentals of the classical electromagnetic theory and electromagnetic material's classification. The classification problem of negative-index materials is discussed.

Chapter 3 presents a general theory of transmission lines in order to include coupled systems as virtual transmission lines and to point out their similarities with physical transmission lines. 
Chapter 4 demonstrates that MTMs can be seen as virtual impedances when coupled to virtual lines and can be used to improve the impedance matching between a virtual line and its terminals.

Chapter 5 demonstrates by means of analytic calculations and numerical simulations that a magnetic link can be satisfactorily described as a VMGTL.

Chapter 6 presents the main conclusions and results obtained during the realization of this work. 


\section{2}

\section{Theoretical framework}

In this chapter, the considered simplifying hypotheses are presented and the fundamentals of classical electromagnetics are reviewed. The physical meaning of the effective electromagnetic properties of materials and their classification problems are presented and discussed. Also, a general classification of materials is proposed in order to include negativeindex ones. Finally, the problem of wave propagation in material media is addressed in order to elucidate the meaning of negative phase velocity.

\section{1.}

\section{Introduction}

For the purpose of this work, the following assumptions are taken:

- The electromagnetic phenomena are time-harmonic fields (of the form $\left.e^{j \omega t}\right)$;

- The existence of magnetic monopoles;

- The considered media are linear around their operating frequency;

- The magnitudes of the fields represent their root-meansquare (rms) values.

Also, the fields and circuit equations are directly presented in their complex spatial form (only as a function of the position).

\section{2.}

\section{Definitions}

The definitions of the main electromagnetic parameters are listed:

- $\boldsymbol{E}$ is the electric intensity $[\mathrm{V} / \mathrm{m}]$

- $\boldsymbol{H}$ is the magnetic intensity $[A / m]$ 
- $\boldsymbol{D}$ is the electric flux density $\left[\mathrm{C} / \mathrm{m}^{2}\right]$

- $\boldsymbol{B}$ is the magnetic flux density $\left[\mathrm{Wb} / \mathrm{m}^{2}\right]$

- $\boldsymbol{J}_{e}$ is the electric current density $\left[\mathrm{A} / \mathrm{m}^{2}\right]$

- $\boldsymbol{J}_{m}$ is the magnetic current density $\left[\mathrm{V} / \mathrm{m}^{2}\right]$

- $\rho_{e}$ is the electric charge density $\left[\mathrm{C} / \mathrm{m}^{3}\right]$

- $\rho_{m}$ is the magnetic charge density $\left[\mathrm{Wb} / \mathrm{m}^{3}\right]$

- $V_{e}$ is the electric potential [V]

- $V_{m}$ is the magnetic potential $[A]$

- $I_{e}$ is the electric current $[A]$

- $I_{m}$ is the magnetic current $[\mathrm{V}]$

- $q_{e}$ is the electric charge $[C]$

- $q_{m}$ is the magnetic charge $[\mathrm{Wb}]$

- $\psi_{e}$ is the electric flux $[C]$

- $\psi_{m}$ is the magnetic flux [Wb]

- $\sigma_{e}$ is the electric conductivity $[S / m]$

- $\sigma_{m}$ is the magnetic conductivity $[\Omega / m]$

- $f$ is the frequency $[\mathrm{Hz}]$

- $\omega$ is the angular frequency $[\mathrm{rad} / \mathrm{s}]$

- $\boldsymbol{k}$ is the wave vector $[\mathrm{rad} / \mathrm{m}]$

\section{3.}

\section{Electromagnetic properties of materials}

A material is called an electric material if it interacts with an incident electric field and presents a characteristic electric response that differs from free space while a material is called a magnetic material if interacts with an incident magnetic field and presents a characteristic magnetic response that differs from free space. In nature, most materials possess an electric response but just a few of them possess a magnetic one.

The EM properties of materials are those properties that govern the rate at which material respond to an applied EM field. The electromagnetic properties can be decoupled into two independent properties: the one governing the rate at which materials respond to an applied electric field, 
the electric permittivity $\varepsilon$, and the one at which materials respond to an applied magnetic field, the magnetic permeability $\mu$.

These EM properties are commonly represented as complex numbers:

$$
\begin{aligned}
& \varepsilon=\varepsilon^{\prime}-j \varepsilon^{\prime \prime}[F / m] \\
& \mu=\mu^{\prime}-j \mu^{\prime \prime}[H / m]
\end{aligned}
$$

where the real part of $\varepsilon$ and $\mu$ - the capacitivity $\varepsilon^{\prime}$ and the inductivity $\mu^{\prime}-$ refers to the rate at which the material stores and emits electric and magnetic energy, respectively, and their imaginary part - the electric loss factor $\varepsilon^{\prime \prime}$ and the magnetic loss factor $\mu^{\prime \prime}$ - refers to the rate at which the material dissipates energy from the fields in form of heat.

If the EM properties depends on $\omega$, the material is said to be dispersive:

$$
\begin{aligned}
& \varepsilon(\omega)=\varepsilon^{\prime}(\omega)-j \varepsilon^{\prime \prime}(\omega) \\
& \mu(\omega)=\mu^{\prime}(\omega)-j \mu^{\prime \prime}(\omega)
\end{aligned}
$$

From a macroscopic point of view, there is no difference between losses associated with free-electrons and losses associated with bound charges in an electric material. So, in general electric losses are represented in terms of effective conductivity or loss tangent [8] [9]:

$$
\tan \delta_{e} \equiv \frac{\varepsilon^{\prime \prime}}{\varepsilon^{\prime}}=\frac{\sigma_{e}}{\omega \varepsilon}
$$

The magnetic losses can be represented in a similar way:

$$
\tan \delta_{m} \equiv \frac{\mu^{\prime \prime}}{\mu^{\prime}}=\frac{\sigma_{m}}{\omega \mu}
$$


For anisotropic materials, the electromagnetic parameters become tensor quantities:

$$
\begin{aligned}
& \overline{\overline{\boldsymbol{\varepsilon}}}=\left[\begin{array}{lll}
\varepsilon_{x x} & \varepsilon_{y x} & \varepsilon_{z x} \\
\varepsilon_{x y} & \varepsilon_{y y} & \varepsilon_{z y} \\
\varepsilon_{x z} & \varepsilon_{y z} & \varepsilon_{z z}
\end{array}\right] \\
& \overline{\overline{\boldsymbol{\mu}}}=\left[\begin{array}{lll}
\mu_{x x} & \mu_{y x} & \mu_{z x} \\
\mu_{x y} & \mu_{y y} & \mu_{z y} \\
\mu_{x z} & \mu_{y z} & \mu_{z z}
\end{array}\right]
\end{aligned}
$$

A robust theory of the EM properties of materials requires a quantum-mechanical description of the EM phenomena. However, once the considered material behavior can be well-represented using classical mechanics, the present study is restricted to classical theory.

\subsection{1.}

\section{Electric properties of materials}

The Bohr model describes matter as composed of atoms with positively charged nuclei and a number of discrete orbiting negatively charged particles, named electrons. In the absence of an external electric field, the molecules of dielectrics are macroscopically neutral: the total negative charge of the electrons is equal to the positive charge of the nuclei. If an external electric field is applied, however, it causes a force to be exerted on each charged particle and results in small displacements of positive and negative charges in opposite directions. Those displacements - small in comparison to the dimensions of the atom - polarize a dielectric material originating an electric dipole moment $\boldsymbol{p}$. The electric moment depends on the separation distance between the positive and the negative charge:

$$
\boldsymbol{p}=q_{e} \boldsymbol{d}[C \cdot m]
$$

where $\boldsymbol{d}$ is the displacement vector from negative to positive charge. 
Some dielectrics possess nonzero electric moment even in the absence of an external polarizing field their molecules are formed of two or more dissimilar atoms. Such molecular structures are called polar molecules. In the absence of an external field, the individual dipoles in a polar dielectric are randomly oriented, resulting in no net dipole moment macroscopically. In the presence of an applied electric field, however, the individual dipoles get aligned with it.

Some dielectric materials with polar molecules can exhibit a permanent electric moment even in the absence of an externally applied electric field. Such materials are called electrets. The polarized molecules in those materials tend to align with the applied field and to be frozen in their new positions after they return to normal temperatures. Permanent polarization remains without an external electric field. Electrets are the electrical equivalents of permanent magnets.

The net electric dipole moment density $\boldsymbol{P}$ extracted from a dielectric material - also known as polarization vector - is the sum of all individual $\boldsymbol{p}$ of its atoms per unit volume:

$$
\boldsymbol{P}=\lim _{\Delta V \rightarrow 0} \frac{1}{\Delta V} \sum \boldsymbol{p}\left[\frac{C}{m^{2}}\right]
$$

$\boldsymbol{P}$ is proportional to the applied electric flux density in free space $\boldsymbol{D}_{\mathbf{0}}$ and to a dimensionless parameter called electric susceptibility $\chi_{e}$ :

$$
\begin{gathered}
\boldsymbol{D}_{0} \triangleq \varepsilon_{0} \boldsymbol{E} \\
\boldsymbol{P}=\chi_{e} \boldsymbol{D}_{0}=\chi_{e} \varepsilon_{0} \boldsymbol{E}
\end{gathered}
$$

For an isotropic medium, the relationship between the electric susceptibility $\chi_{e}$, the polarization vector $\boldsymbol{P}$ and the electric flux density $\boldsymbol{D}$ is given by:

$$
\boldsymbol{D}=\varepsilon_{0} \boldsymbol{E}+\boldsymbol{P}=\varepsilon_{0}\left(1+\chi_{e}\right) \boldsymbol{E}=\varepsilon_{0} \varepsilon_{r} \boldsymbol{E}
$$




$$
\begin{gathered}
\varepsilon_{r} \triangleq 1+\chi_{e} \\
\varepsilon_{0} \cong 8.85 \cdot 10^{-12}\left[\frac{F}{m}\right]
\end{gathered}
$$

where $\varepsilon_{0}$ is the free-space permittivity, $\varepsilon_{r}$ is a dimensionless parameter called relative permittivity.

$$
\begin{gathered}
\varepsilon=\varepsilon_{0} \varepsilon_{r}=\varepsilon^{\prime}-j \varepsilon^{\prime \prime} \\
\varepsilon_{r}=1+\chi_{e}=1+\chi_{e}^{\prime}-j \chi_{e}^{\prime \prime} \\
\therefore \varepsilon^{\prime}=\varepsilon_{0}\left(1+\chi_{e}^{\prime}\right) \\
\varepsilon^{\prime \prime}=\varepsilon_{0} \chi_{e}^{\prime \prime}
\end{gathered}
$$

where $\chi_{e}^{\prime}$ accounts for the real part of $\chi_{e}$, and $\chi_{e}^{\prime \prime}$, for its imaginary part.

The necessary conditions to obtain $\varepsilon^{\prime}<0$ are:

$$
\chi_{e}^{\prime}<0 \text { and }\left|\chi_{e}^{\prime}\right|>1
$$

Considering that the polarization vector $\boldsymbol{P}$ is proportional to the electric susceptibility $\chi_{e}$, a negative $\chi_{e}^{\prime}$ indicates a material very negatively polarized.

\subsection{2.}

\section{Magnetic properties of materials}

The orbiting electrons of the atoms are equivalent to circulating currents and form microscopic magnetic dipoles called orbital magnetic dipole moment of the electron $\boldsymbol{m}_{\boldsymbol{o}}$. In addition, both the electrons and the nuclei of the atoms rotate on their own axes and also present magnetic dipole moments whose sum is the total spin magnetic dipole moment $\boldsymbol{m}_{s}$ of the atom (see Figure 2.1). 


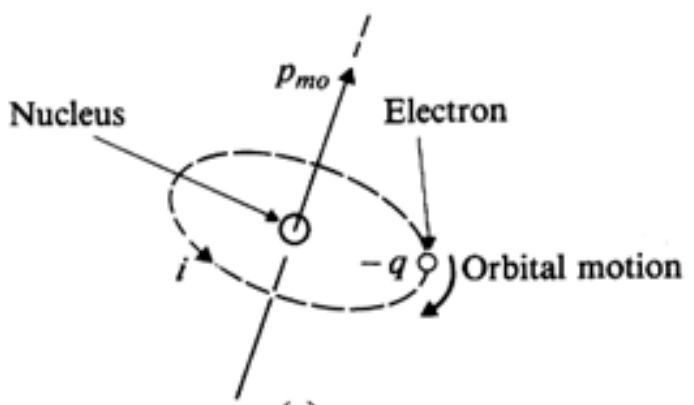

(a)

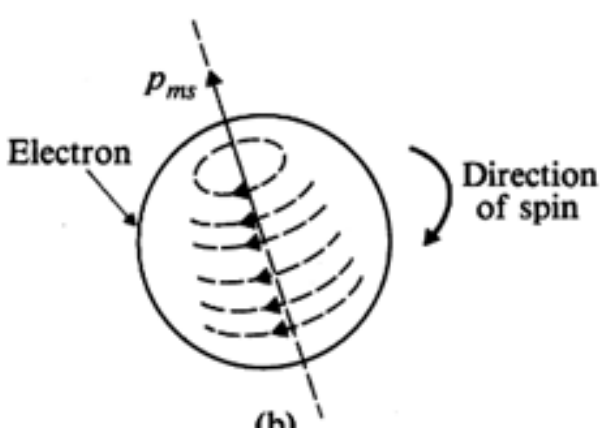

(b)

Figure 2.1: (a) Orbital motion and (b) spin of an electron

Considering that the nucleus of the atom presents much higher mass and then much lower angular velocity $\omega$ than its electrons, the spin magnetic moment of the atom may be neglected. Hence, the magnetic moment $\boldsymbol{m}$ of the atoms relies basically on the sum of the orbital and spin moments of the electrons:

$$
\boldsymbol{m}=\boldsymbol{m}_{o}+\boldsymbol{m}_{s}
$$

For materials with very symmetric atomic shell, the spin and orbital moments opposes to each other $(\boldsymbol{m}=0)$. In the presence of an external field, the orbital moments, analogous to circulating currents, get aligned with applied field, tending to enhance the magnetic flux. In order to keep the magnetic flux constant, as stated by Lenz's law of EM induction, the induced magnetic moment opposes the magnetic moment imposed by the external field, which reduces the velocity of the electrons. Since the spin moment becomes greater than the orbital one $(\boldsymbol{m}<0)$, the resultant net magnetic dipole moment is negative $\left(\sum \boldsymbol{m}<0\right)$. Those materials are called diamagnetic.

For materials with asymmetric atomic shell, the magnetic moments do not cancel each other out $(\boldsymbol{m}>0)$. In the absence of an external magnetic field, the individual $\boldsymbol{m}$ of each atom is randomly oriented, which results in no apparent magnetic behavior of the material on the macroscopic scale. In the presence of an external source, however, all the 
$\boldsymbol{m}$ of the material get aligned with the applied magnetic field, enhancing the magnetic flux density. That behavior is macroscopically described as a positive net magnetic dipole moment $\left(\sum \boldsymbol{m}>0\right)$. Those materials are called paramagnetic.

In materials capable of form magnetic domains, where the magnetic moments are positive and locally aligned, the paramagnetic behavior becomes strong. Those materials are called ferromagnetic in the literature and can be permanently magnetized (magnets).

The net magnetic dipole moment density $\boldsymbol{M}$ extracted from a magnetic material - also known as magnetization vector - is the sum of all individual $\boldsymbol{m}$ of its atoms per unit volume (see Figure 2.2):

$$
\boldsymbol{M}=\lim _{\Delta V \rightarrow 0} \frac{1}{\Delta V} \sum \boldsymbol{m}\left[\frac{A}{m}\right]
$$

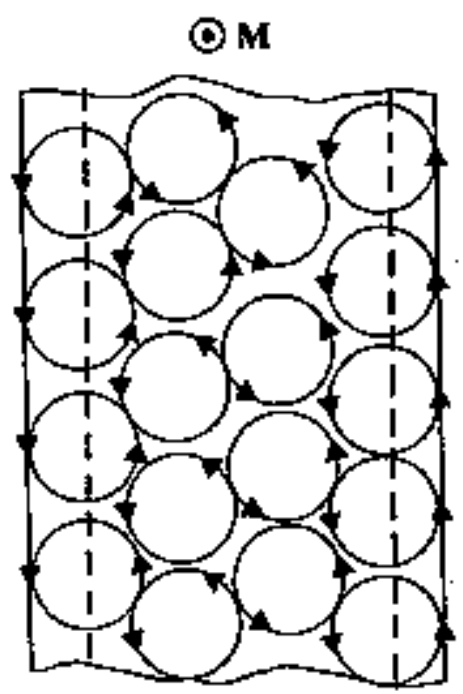

Figure 2.2: Cross section of a magnetized material

$\boldsymbol{M}$ is proportional to the applied magnetic field $\boldsymbol{H}$ and to a dimensionless parameter called magnetic susceptibility $\chi_{m}$ :

$$
\boldsymbol{M}=\chi_{m} \boldsymbol{H}
$$


For an isotropic medium, the relationship between the magnetic susceptibility $\chi_{m}$, the magnetization vector $\boldsymbol{M}$ and the magnetic flux density $\boldsymbol{B}$ is given by:

$$
\begin{gathered}
\boldsymbol{B}=\mu_{0}(\boldsymbol{H}+\boldsymbol{M})=\mu_{0}\left(1+\chi_{m}\right) \boldsymbol{H}=\mu_{0} \mu_{r} \boldsymbol{H} \\
\mu_{r} \triangleq 1+\chi_{m} \\
\mu_{0} \equiv 4 \pi \cdot 10^{-7}\left[\frac{H}{m}\right]
\end{gathered}
$$

where $\mu_{0}$ is the free-space permeability, $\mu_{r}$ is a dimensionless parameter called relative permeability.

$$
\begin{gathered}
\mu=\mu_{0} \mu_{r}=\mu^{\prime}-j \mu^{\prime \prime} \\
\mu_{r}=1+\chi_{m}=1+\chi_{m}^{\prime}-j \chi_{m}^{\prime \prime} \\
\therefore \mu^{\prime}=\mu_{0}\left(1+\chi_{m}^{\prime}\right) \\
\mu^{\prime \prime}=\mu_{0} \chi_{m}^{\prime \prime}
\end{gathered}
$$

where $\chi_{m}^{\prime}$ accounts for the real part of $\chi_{m}$, and $\chi_{m}^{\prime \prime}$, for its imaginary part. The necessary conditions to obtain $\mu^{\prime}<0$ are:

$$
\chi_{m}^{\prime}<0 \text { and }\left|\chi_{m}^{\prime}\right|>1
$$

Considering that the magnetization vector $\boldsymbol{M}$ is proportional to the magnetic susceptibility $\chi_{m}$, a negative $\chi_{m}^{\prime}$ indicates a material very negatively magnetized.

The main characteristic of materials presenting diamagnetic response is that their individual atoms possess no intrinsic magnetic moment $(\boldsymbol{m}=0)$, then the material reacts to an applied field opposing it in order to prevent the enhancement of the magnetic flux accordingly to the 
law of electromagnetic induction. For most materials, however, the diamagnetic effect is very weak, implying on slightly negative $\chi_{m}^{\prime}$.

The behavior of $\mu$-negative materials is diamagnetic-like but much stronger. They are highly magnetized by induction effect, causing their potential energy to be inverted in relation to the potential of the magnetic field. When the potential of a $\mu$-negative material is greater than the potential of the incoming field, the material is more energetic than the field, which inverts the direction of the energy flux (the material sends energy to the magnetic field). For a passive structure, it implies that the net magnetic field at the interface with the material will be zero. Hence, $\mu$-negative materials can be classified as diamagnetic materials with high density of inductive currents in order to achieve very negative $\chi_{m}^{\prime}$.

\section{4 .}

\section{Physical interpretation of negative-index materials}

The EM response of the material varies depending on the state it finds itself. The four fundamental states of matter (the ones which are observable in everyday life) are: solid, liquid, gas and plasma. For each one of them, the atomic density package, relative temperature of the particles and their degrees of freedom change.

In solids, particles are closely packed then the bonding forces between them are so strong that they cannot move freely but only vibrate presenting fixed volume and shape. In liquids, particles are free to move around each other but the bonding forces keeping them together are still strong so they can vary in shape but with a fixed volume. In gases, the bonding forces are weak so they vary in both volume and shape. Finally, plasmas are very much like a gas, with no fixed volume or shape, but electrically charged (although the plasma's overall charge is zero, its positive and negative particles are mostly unbounded making it an ionized gas).

A gas becomes plasma when it exposed to extremely high temperatures or it experiences a huge difference of electric potential between two points. In plasmas, despite the fact the charged particles are 
unbounded they respond collectively to any source of electromagnetic field. Each charged particle affects and is affected by the movement of other charged particles. This collective behavior is measure in terms of plasma oscillations. If the plasma frequency is large compared to the collision frequency of the charged particles with the neutral ones then electrostatic interactions (or magnetostatic interactions, if magnetic charges are taken into consideration) rule over the ordinary gas kinetics. The other conditions to achieve the plasma state are:

- A charge particle interacts with many nearby charge carriers rather than just interacting with the closest charge carrier (collective behavior);

- The interactions in the bulk of the plasma are more important than those at its edges. If the boundary effects are negligible, hence the quasineutral condition of the plasma is satisfied. 

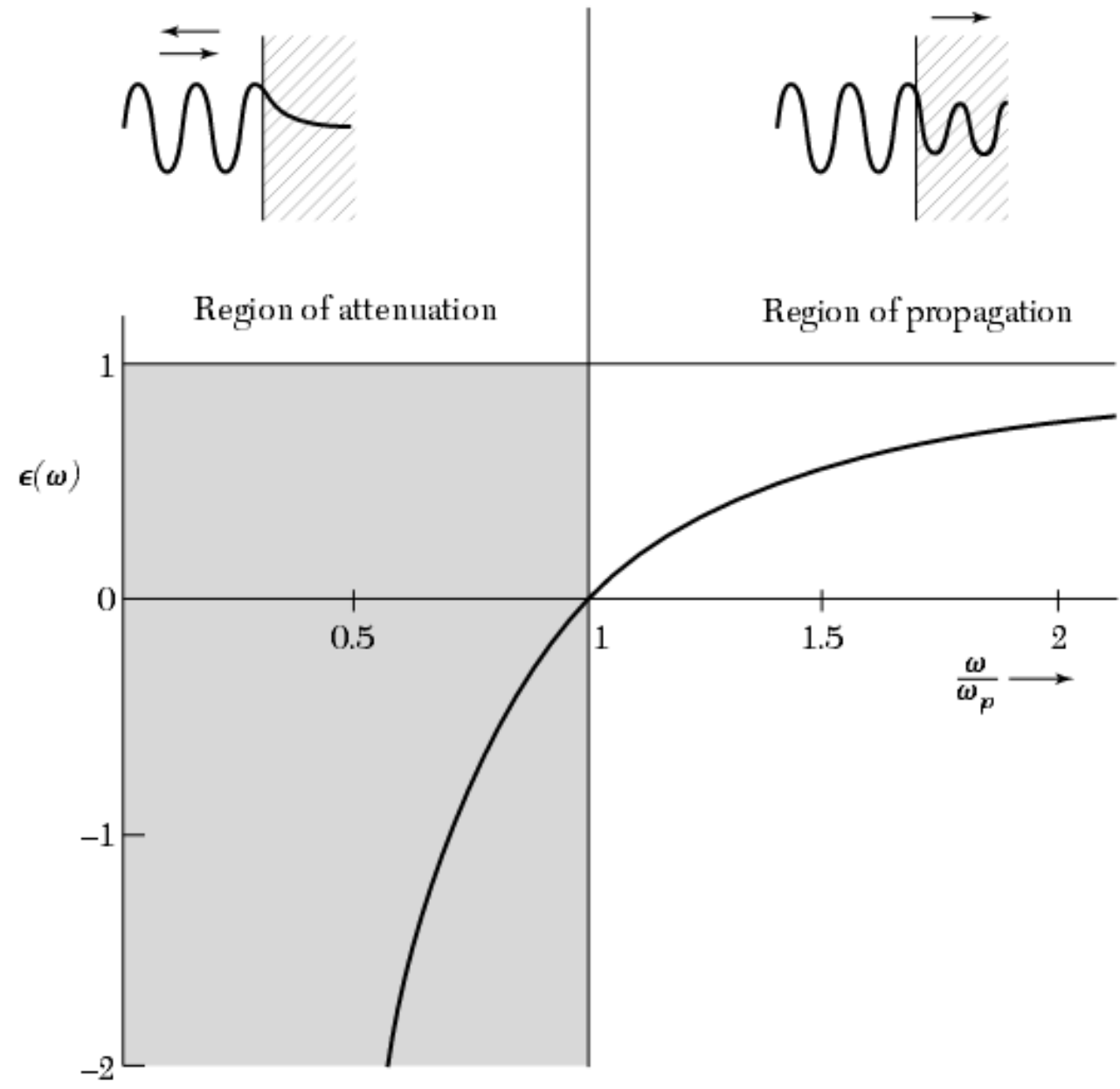

Figure 2.3: Dielectric function of a plasma. Propagation is only possible above the plasma frequency $\omega_{p}$. EM wave is reflected when $\varepsilon^{\prime}$ is negative [10]

The quantization of plasma oscillations results in quasiparticles called plasmons.

It is known that electric plasmas are described by a permittivity function whose real part $\varepsilon^{\prime}$ assumes negative values below the plasma frequency (see Figure 2.3) [10].

Nonetheless, other materials such as metals can also be described as presenting negative $\varepsilon^{\prime}$ below a certain range. Due to the free, delocalized electrons in their structures, metallic crystals behave as positively charged atomic cores immersed in a oscillating "electron cloud", which is equivalent to an electric plasma.

For most metals, the plasma frequency is subluminal (only silver and other noble metals present plasmatic behavior at optical frequencies). By 
analogy, since materials with $\varepsilon^{\prime}<0$ are electric plasma, materials with $\mu^{\prime}<0$ must be magnetic plasma. Considering that electric plasmas are very good electrical conductors, magnetic plasmas must be good magnetic conductors.

For example, if an electric field is applied to a material such as glass - which presents positive $\varepsilon^{\prime}$ - the energy carried by the field will be partial or totally stored in the material. On the contrary, if the same electric field is applied to an electric conductor such as metal sheet - which presents negative $\varepsilon^{\prime}$ - no energy in the electric form will be received by the material causing the energy delivered by the field to be reabsorbed into it, implying zero divergence of the electric field at this point. That is why an electric field at a metallic surface is virtually zero. The same reasoning is valid for the magnetic field and magnetic materials and conductors.

Another characteristic of negative-index materials (NIM) is the conversion of the form of energy: $\varepsilon$-negative materials convert the electric field into the magnetic one while $\mu$-negative materials convert the magnetic field into the electric one. For example, when an incident electric field arrives at an $\varepsilon$-negative surface (e.g., metals) it generates an electric current on it, which is source of magnetic field.

Then, there is nothing out of place or spectacular concerning negative EM properties. They are a comprehensible and regular form of the field-material interaction. So, $\varepsilon^{\prime}<0$ and $\mu^{\prime}<0$ can be interpreted as forbidden states of energy storage in the electric and magnetic form, respectively. And their magnitude can be seen as the rate at which the material is converting the incident field in a current source of its complementary field.

Finally, it is important to mention the class of materials that present $\left|\varepsilon_{r}^{\prime}\right|<1$ or $\left|\mu_{r}^{\prime}\right|<1$. As shown in [11], such materials do not represent a new class but represent media where the damping factor is dominant. In other words, they are "resistive materials". 


\section{5.}

\section{Classification of electromagnetic materials}

Based on the previous discussion on the behavior of positive and negative-index materials, the EM materials can be classified according to Table 1.

\begin{tabular}{|c|c|c|c|}
\hline \multicolumn{2}{|c|}{ Electric materials } & \multicolumn{2}{c|}{ Magnetic materials } \\
\hline$\varepsilon_{r}^{\prime}<0$ & Electric conductor & $\mu_{r}^{\prime}<0$ & Magnetic conductor \\
\hline $0 \leq \varepsilon_{r}^{\prime}<1$ & Sub-electric & $0 \leq \mu_{r}^{\prime}<1$ & Sub-magnetic \\
\hline$\varepsilon_{r}^{\prime}=1$ & Non-electric & $\mu_{r}^{\prime}=1$ & Non-magnetic \\
\hline$\varepsilon_{r}^{\prime}>1$ & Electric & $\mu_{r}^{\prime}>1$ & Magnetic \\
\hline
\end{tabular}

Table 1: Proposed classification

For historical reasons, electric materials are usually called dielectrics in the literature. This nomenclature problem comes from the fact that in the beginning of the modern studies of electromagnetism (by the time of Volta, Coulomb and Ampère) researchers believed that the "electric energy" was contained and carried by the "electric charges". The materials where those electric charges could be forced to propagate (generating "electricity" or movement of electric charges) were named "conductors" and the ones where they could not propagate were named "insulators" or "dielectrics" (non-electrics since $d i$ is a greek prefix that means non). Later, Faraday demonstrated that the so-called "electric energy" could be transmitted "in the air" without any electric conductor connecting the source and the load by means of a time-varying magnetic field. In those "magnetic circuits", the materials where the "magnetic flux" flew very easily where named "magnetic" while those where it flew with great difficulty were called "nonmagnetic". Based on Faraday's work, Maxwell solved the problem of the electric current's continuity in a capacitor and developed the classical theory of electromagnetism, showing that electric and magnetic field were actually two aspect of the same entity, the EM field, and that the very mechanism of electric energy transmission is the "EM wave".

Despite these well-known facts, electric materials are still classified in terms of their conductibility of "electric currents" while magnetic materials 
are classified in terms of their reluctance to "magnetic fluxes". This is why the classical classification of the EM materials is sometimes confusing.

\section{6.}

Fundamental concepts of electromagnetic field theory

\subsection{1.}

\section{Basic field and circuit equations}

\begin{tabular}{|c|c|c|}
\hline & Differential form & Integral form \\
\hline Gauss's law & $\nabla \cdot \boldsymbol{D}=\rho_{e}$ & $\oiint \boldsymbol{D} \cdot d \boldsymbol{s}=\iiint \rho_{e} d V$ \\
\hline $\begin{array}{c}\text { Gauss's law for } \\
\text { magnetism }\end{array}$ & $\nabla \cdot \boldsymbol{B}=\rho_{m}$ & $\oiint \boldsymbol{B} \cdot d \boldsymbol{s}=\iiint \rho_{m} d V$ \\
\hline $\begin{array}{c}\text { Maxwell-Faraday- } \\
\text { Lenz's law }\end{array}$ & $-\nabla \times \boldsymbol{E}=\boldsymbol{J}_{m}$ & $-\oint \boldsymbol{E} \cdot d \boldsymbol{l}=\iint \boldsymbol{J}_{m} d \boldsymbol{s}$ \\
\hline $\begin{array}{c}\text { Maxwell-Ampère's } \\
\text { law }\end{array}$ & $\nabla \times \boldsymbol{H}=\boldsymbol{J}_{e}$ & $\oint \boldsymbol{H} \cdot d \boldsymbol{l}=\iint \boldsymbol{J}_{e} d \boldsymbol{s}$ \\
\hline
\end{tabular}

Table 2: Maxwell equations

\begin{tabular}{|c|c|c|}
\hline & Differential form & Integral form \\
\hline $\begin{array}{c}\text { Electric } \\
\text { current }\end{array}$ & $\nabla \cdot \boldsymbol{J}_{e}=-j \omega \rho_{e}$ & $\oiint \boldsymbol{J}_{e} \cdot d \boldsymbol{s}=-j \omega \iiint \rho_{e} d V$ \\
\hline $\begin{array}{c}\text { Magnetic } \\
\text { current }\end{array}$ & $\nabla \cdot \boldsymbol{J}_{m}=-j \omega \rho_{m}$ & $\oiint \boldsymbol{J}_{m} \cdot d \boldsymbol{s}=-j \omega \iiint \rho_{m} d V$ \\
\hline
\end{tabular}

Table 3: Current continuity equations

\begin{tabular}{|c|c|}
\hline Electric circuit & Magnetic circuit \\
\hline$V_{e}=\int \boldsymbol{E} \cdot d \boldsymbol{l}$ & $V_{m}=\int \boldsymbol{H} \cdot d \boldsymbol{l}$ \\
\hline$I_{e}=\iint \boldsymbol{J}_{e} \cdot d \boldsymbol{s}$ & $I_{m}=\iint \boldsymbol{J}_{m} \cdot d \boldsymbol{s}$ \\
\hline$\psi_{e}=\iint \boldsymbol{D} \cdot d \boldsymbol{s}$ & $\psi_{m}=\iint \boldsymbol{B} \cdot d \boldsymbol{s}$ \\
\hline$q_{e}=\iiint \rho_{e} d V$ & $q_{m}=\iiint \rho_{m} d V$ \\
\hline
\end{tabular}

Table 4: Conversion of field equations to circuit ones 


\begin{tabular}{|c|c|}
\hline Electric flux density & $\boldsymbol{D}=\boldsymbol{\varepsilon} \boldsymbol{E}\left[\frac{C}{m^{2}}\right]$ \\
\hline Magnetic flux density & $\boldsymbol{B}=\boldsymbol{\mu} \boldsymbol{H}\left[\frac{W b}{m^{2}}\right]$ \\
\hline $\begin{array}{c}\text { Electric conduction } \\
\text { current density }\end{array}$ & $\boldsymbol{J}_{e, c}=\sigma_{e} \boldsymbol{E}\left[\frac{A}{m^{2}}\right]$ \\
\hline $\begin{array}{c}\text { Electric displacement } \\
\text { current density }\end{array}$ & $\boldsymbol{J}_{e, d}=j \omega \boldsymbol{D}\left[\frac{A}{m^{2}}\right]$ \\
\hline $\begin{array}{c}\text { Total electric current } \\
\text { density }\end{array}$ & $\boldsymbol{J}_{e}=\boldsymbol{J}_{e, c}+\boldsymbol{J}_{e, d}\left[\frac{A}{m^{2}}\right]$ \\
\hline $\begin{array}{c}\text { Magnetic conduction } \\
\text { current density }\end{array}$ & $\boldsymbol{J}_{m, c}=\sigma_{m} \boldsymbol{H}\left[\frac{V}{m^{2}}\right]$ \\
\hline $\begin{array}{c}\text { Magnetic displacement } \\
\text { current density }\end{array}$ & $\boldsymbol{J}_{m, d}=j \omega \boldsymbol{B}\left[\frac{V}{m^{2}}\right]$ \\
\hline $\begin{array}{c}\text { Total magnetic current } \\
\text { density }\end{array}$ & $\boldsymbol{J}_{m}=\boldsymbol{J}_{m, c}+\boldsymbol{J}_{m, d}\left[\frac{V}{m^{2}}\right]$ \\
\hline
\end{tabular}

Table 5: Constitutive relationships

\subsection{2.}

\section{Travelling EM wave}

In sourceless region such as the free space, EM field can transport energy in the form of waves [8] [9]. For a time-harmonic EM field, the general solution for the EM wave propagation in the canonical base is given by:

$$
\begin{gathered}
\boldsymbol{E}(z)=\left(E_{0}^{+} e^{-k z} \pm E_{0}^{-} e^{k z}\right) \hat{x} \\
\boldsymbol{H}(z)=\left(H_{0}^{+} e^{-k z} \pm H_{0}^{-} e^{k z}\right) \hat{y}
\end{gathered}
$$

The wave vector $\boldsymbol{k}$ is defined in terms of EM properties as:

$$
\boldsymbol{k}=\omega \sqrt{\boldsymbol{\varepsilon} \boldsymbol{\mu}}
$$


The wave impedance $Z_{w}$ is defined as:

$$
Z_{w}=\frac{\boldsymbol{E}}{\boldsymbol{H}}
$$

The phase velocity of the wave is given by:

$$
v_{p}=\frac{\omega}{\boldsymbol{k}}
$$

And the group velocity:

$$
v_{g}=\frac{\partial \omega}{\partial \boldsymbol{k}}
$$

\subsection{3.}

\section{Power and energy in materials}

The ability of a material to store energy in the electric form (electric energy) is proportional to its capacitance $C$ while its ability to store energy in the magnetic form (magnetic energy) is proportional to its inductance $L$. The relationship between the energy storage ability of a material and its EM parameters is given by:

$$
\begin{aligned}
& C=\varepsilon^{\prime} g_{c}[F] \\
& L=\mu^{\prime} g_{l}[H]
\end{aligned}
$$

where $g_{c}$ and $g_{l}$ are geometric functions with unit meter that depend on the shape of the capacitor and the inductor, respectively.

Based on the previous definitions, the energy density, the total stored energy and dissipated power in the material can be determined: 


\begin{tabular}{|c|c|}
\hline $\begin{array}{c}\text { Time-average electric } \\
\text { energy density }\end{array}$ & $\bar{u}_{e}=\operatorname{Re}\left\{\frac{\boldsymbol{D} \cdot \boldsymbol{E}}{2}\right\}=\frac{\varepsilon^{\prime} \cdot \boldsymbol{E}^{2}}{2}\left[\frac{\mathrm{J}}{\mathrm{m}^{3}}\right]$ \\
\hline $\begin{array}{c}\text { Time-average magnetic } \\
\text { energy density }\end{array}$ & $\bar{u}_{m}=\operatorname{Re}\left\{\frac{\boldsymbol{B} \cdot \boldsymbol{H}}{2}\right\}=\frac{\mu^{\prime} \cdot \boldsymbol{H}^{2}}{2}\left[\frac{\mathrm{J}}{\mathrm{m}^{3}}\right]$ \\
\hline $\begin{array}{c}\text { Time-average energy } \\
\text { density }\end{array}$ & $\bar{u}=\bar{u}_{e}+\bar{u}_{m}\left[\frac{\mathrm{J}}{\mathrm{m}^{3}}\right]$ \\
\hline
\end{tabular}

Table 6: Energy density

\begin{tabular}{|c|c|}
\hline $\begin{array}{c}\text { Heat } \\
\text { dissipation } \\
\text { density }\end{array}$ & $p_{d, c}=\operatorname{Re}\left\{\boldsymbol{E} \cdot \boldsymbol{J}_{e, c}^{*}+\boldsymbol{H}^{*} \cdot \boldsymbol{J}_{m, c}\right\}=\sigma_{e} \boldsymbol{E}^{2}+\sigma_{m} \boldsymbol{H}^{2}\left[\frac{W}{m^{3}}\right]$ \\
\hline $\begin{array}{c}\text { Material loss } \\
\text { density }\end{array}$ & $p_{d, d}=\operatorname{Re}\left\{\boldsymbol{E} \cdot \boldsymbol{J}_{e, d}^{*}+\boldsymbol{H}^{*} \cdot \boldsymbol{J}_{m, d}\right\}=\omega \varepsilon^{\prime \prime} \boldsymbol{E}^{2}+\omega \mu^{\prime \prime} \boldsymbol{H}^{2}\left[\frac{W}{m^{3}}\right]$ \\
\hline $\begin{array}{c}\text { Total power } \\
\text { dissipation } \\
\text { density }\end{array}$ & $p_{d} \triangleq p_{d, c}+p_{d, d}\left[\frac{W}{m^{3}}\right]$ \\
\hline
\end{tabular}

Table 7: Power dissipation density

\begin{tabular}{|c|c|}
\hline $\begin{array}{c}\text { Time-average stored } \\
\text { electric energy }\end{array}$ & $\bar{U}_{e}=\frac{1}{2} \iiint \bar{u}_{e} d V=\frac{C V_{e}^{2}}{2}+\frac{C I_{m}^{2}}{2}[J]$ \\
\hline $\begin{array}{c}\text { Time-average stored } \\
\text { magnetic energy }\end{array}$ & $\bar{U}_{m}=\frac{1}{2} \iiint \bar{u}_{m} d V=\frac{L I_{e}^{2}}{2}+\frac{L V_{m}^{2}}{2}[J]$ \\
\hline Total stored energy & $\bar{U}=\bar{U}_{e}+\bar{U}_{m}[J]$ \\
\hline
\end{tabular}

Table 8: Stored energy

The total power dissipated by the material is obtained from:

$$
P_{d}=\iiint p_{d} d V[W]
$$

And the total energy flux density going through the material's surface is given by: 


$$
\boldsymbol{S}=\boldsymbol{E} \times \boldsymbol{H}^{*}\left[\frac{V A}{m^{2}}\right]
$$

where $\boldsymbol{S}$ is the Poynting vector.

The time-average energy flux density going through the material's surface is given by:

$$
\overline{\boldsymbol{S}}=\operatorname{Re}\left\{\boldsymbol{E} \times \boldsymbol{H}^{*}\right\}\left[\frac{W}{m^{2}}\right]
$$

The complex power flow density leaving a region can be expressed by:

$$
p_{f} \triangleq \nabla \cdot S\left[\frac{V A}{m^{3}}\right]
$$

If sources are present, the complex power density supplied by the sources can be defined as:

$$
p_{s}=-\left(\boldsymbol{E} \cdot \boldsymbol{J}_{e, i}^{*}+\boldsymbol{H}^{*} \cdot \boldsymbol{J}_{m, i}\right)\left[\frac{V A}{m^{3}}\right]
$$

where $\boldsymbol{J}_{e, i}$ and $\boldsymbol{J}_{m, i}$ are the currents impressed by the sources.

The Poynting's theorem states that "the decrease in the EM energy per unit time in a certain volume is equal to the sum of the work done by the field forces and the net outward flux per unit time" [12]:

$$
p_{s}=p_{f}+p_{d}+j 2 \omega\left(\bar{u}_{m}-\bar{u}_{e}\right)\left[\frac{V A}{m^{3}}\right]
$$

By integrating throughout the region and using the divergence theorem, the time-average exiting complex power is obtained:

$$
P_{f}=\iiint \nabla \cdot \boldsymbol{S} d V=\oiint \boldsymbol{S} \cdot d \boldsymbol{s}=\oiint \boldsymbol{E} \times \boldsymbol{H}^{*} d \boldsymbol{s}[V A]
$$


As well as the time-average complex power balance:

$$
P_{s}=P_{f}+P_{d}+j 2 \omega\left(\bar{U}_{m}-\bar{U}_{e}\right)[V A]
$$

where the real part of $P_{f}$ represents the time-average power flow leaving a region (in the form of propagating waves) and its imaginary part represents the time-average reactive power leaving a region (in the form of evanescent - non-propagating - waves):

$$
\begin{gathered}
P=\operatorname{Re}\left\{P_{f}\right\}=\operatorname{Re}\left\{P_{s}\right\}-P_{d}[W] \\
Q=\operatorname{Im}\left\{P_{f}\right\}=\operatorname{Im}\left\{P_{s}\right\}+j 2 \omega\left(\bar{U}_{e}-\bar{U}_{m}\right)[V A R]
\end{gathered}
$$

\section{7.}

\section{Velocity of power transportation}

In non-dispersive medium, the velocity of power transportation or the signal velocity $v_{s}$ is equal to the phase velocity $v_{p}$ of the EM wave.

In a dispersive and time-invariant medium, $v_{s}$ is given by the group velocity $v_{g}$, which is the velocity of the waveform of travelling EM wave.

If the medium is dispersive and time-variant, the waveform has no constant shape, so $v_{g}$ differs from the velocity at which the power is conveyed by the EM field [13]. In that case, $v_{s}$ is derived directly from the time-average energy flux density $\overline{\boldsymbol{S}}$ and the time-average energy density of the medium $\bar{u}$ :

$$
v_{s}=\frac{\overline{\boldsymbol{S}}}{\bar{u}} \leq c
$$

where $c$ is the velocity of light in the vacuum. 


\section{8.}

\section{Causality of negative-index materials}

The entropy condition for NIMs can only be satisfied if the following conditions are attended [14] [15]:

$$
\frac{\partial\left(\omega \varepsilon^{\prime}\right)}{\partial \omega}>0 \text { and } \frac{\partial\left(\omega \mu^{\prime}\right)}{\partial \omega}>0, \forall \omega
$$

Otherwise, such materials would store "negative energy", which is a non-causal system (not realizable).

\begin{tabular}{|c|c|}
\hline $\begin{array}{c}\text { Time-average electric } \\
\text { energy density }\end{array}$ & $\bar{u}_{e}=\frac{1}{2} \frac{\partial\left(\omega \varepsilon^{\prime}\right)}{\partial \omega} \cdot \boldsymbol{E}^{2}$ \\
\hline $\begin{array}{c}\text { Time-average magnetic } \\
\text { energy density }\end{array}$ & $\bar{u}_{m}=\frac{1}{2} \frac{\partial\left(\omega \mu^{\prime}\right)}{\partial \omega} \cdot \boldsymbol{H}^{2}$ \\
\hline $\begin{array}{c}\text { Time-average stored } \\
\text { electric energy }\end{array}$ & $\bar{U}_{e}=\frac{1}{2} \frac{\partial(\omega C)}{\partial \omega} V_{e}^{2}+\frac{1}{2} \frac{\partial(\omega C)}{\partial \omega} I_{m}^{2}$ \\
\hline $\begin{array}{c}\text { Time-average stored } \\
\text { magnetic energy }\end{array}$ & $\bar{U}_{m}=\frac{1}{2} \frac{\partial(\omega L)}{\partial \omega} I_{e}^{2}+\frac{1}{2} \frac{\partial(\omega L)}{\partial \omega} V_{m}^{2}$ \\
\hline
\end{tabular}

Table 9: Energy density and stored energy in negative-index materials

Therefore, negative EM properties are only realizable if the material is highly dispersive.

\section{9.}

\section{Wave propagation in material media}

The propagation of an EM wave can be interpreted as a periodic exchange of energy between the medium and the EM field [16]. The stored energy in the medium on the back side of the wave packet is transformed into field energy and transported to the front side of the wave packet where it is reabsorbed and stored again in the medium.

The velocity at which the fields transport energy by converting the stored energy into field energy and back is the phase velocity $v_{p}$, which is 
an apparent velocity. Defining $v_{m}$ as the velocity at which the material transports energy due to the oscillatory behavior of its dipoles (in the sense of the Lorentz model), $\bar{u}_{f}$ as the time-average energy density of the EM field and $\bar{u}_{\text {mat }}$ as the time-average energy density of the material, the total energy flux density $\overline{\boldsymbol{S}}$ can be rewritten as the energy flux density due to the oscillating EM fields minus the energy flux density due to the oscillating dipoles in the material:

$$
\begin{gathered}
\overline{\boldsymbol{S}}(\omega)=v_{s}(\omega) \bar{u}(\omega)=v_{p}(\omega) \bar{u}_{f}(\omega)-v_{m}(\omega) \bar{u}_{m a t}(\omega) \\
\bar{u}(\omega)=\bar{u}_{f}(\omega)+\bar{u}_{m a t}(\omega) \\
v_{p}(\omega)=\frac{v_{s}(\omega) \bar{u}(\omega)+v_{m}(\omega) \bar{u}_{m a t}(\omega)}{\bar{u}_{f}(\omega)} \\
\therefore v_{p}(\omega)=v_{s}(\omega)+\frac{\left(v_{s}(\omega)+v_{m}(\omega)\right) \bar{u}_{m a t}(\omega)}{\bar{u}_{f}(\omega)}
\end{gathered}
$$

Eq. (52) is postulated based on the fact that the dipoles that are formed by polarization or magnetization of the material take their energy from the fields. So, the energy flux density of the fields leaving the region must be diminished from the one associated with the motion of the dipoles.

As mentioned before, a medium is called non-dispersive if EM properties are not frequency dependent. The only medium that is truly non-dispersive (in the entire frequency spectrum) is the fundamental medium, the EM field in the proper sense. Any other medium arises from the interaction of the EM field with the particles of condensed matter. Thus, the non-dispersive medium is equivalent to the EM field in a perfectly source-free and sink-free region. Since there is no material properly speaking in the non-dispersive case (EM field is the sole medium):

$$
\begin{gathered}
\bar{u}(\omega)=\bar{u}_{f}(\omega) \rightarrow \bar{u}_{\text {mat }}(\omega)=0 \\
v_{p}(\omega)=v_{s}(\omega)
\end{gathered}
$$


As expected, $v_{p}$ and $v_{s}$ are exactly the same for all frequencies.

However, in a region of space with charged particles, energy can be locally stored or dissipated. The resulting interaction between matter and fields becomes frequency dependent, causing the phenomenon of dispersion.

In normal dispersion, or positive dispersion, power is irreversibly taken from the EM field and stored into the material $\left(\bar{u}_{m a t}>0\right)$. Assuming the simplifying hypothesis that $v_{m}$ is much slower than $v_{s}$ :

$$
\begin{gathered}
v_{p}(\omega) \approx v_{s}(\omega)\left(1+\frac{\bar{u}_{m a t}(\omega)}{\bar{u}_{f}(\omega)}\right)=v_{s}\left(\frac{\bar{u}(\omega)}{\bar{u}_{f}(\omega)}\right) \\
\bar{u}_{m a t}>0 \rightarrow \bar{u}_{f}<\bar{u} \\
\bar{u}_{f} \leq \bar{u} \rightarrow v_{p} \geq v_{s}
\end{gathered}
$$

So, in accordance with EM theory, Eq. (55) predicts that $v_{p}$ is greater than $v_{s}$ in normal dispersive media.

In anomalous dispersion, or negative dispersion, which occurs around the resonance of the material $\omega_{0}, v_{m}$ becomes much greater than $v_{s}$ :

$$
\left|v_{m}\right| \gg\left|v_{s}\right| \rightarrow \bar{u}_{m a t}>\bar{u}_{f}
$$

In this situation, the medium is said to be excited or in nonequilibrium $\left(\bar{u}_{m a t}>\bar{u}_{f}>0\right)$. Instead of net power being transported from the wave front into the medium as in normal dispersion, it is the front wave that extracts net power from the material $\left(v_{m}<0\right)$, by reducing the excitation of the system back to the equilibrium state $\left(\bar{u}_{m a t} \leq \bar{u}_{f}\right)$.

The relaxation of the excited medium implies that the first temporal derivative of its stored energy is negative: 


$$
\dot{\bar{U}}_{\text {mat }}\left(\omega_{0}\right)<0
$$

Knowing that:

$$
\dot{V}=\oiint v_{m} d \boldsymbol{s}\left[\frac{m^{3}}{s}\right]
$$

where $\dot{V}$ is the volumetric flow rate of the energy through the surface of the material.

The energy density can be written as:

$$
\bar{u}_{m a t} \equiv \frac{\dot{\bar{U}}_{m a t}}{\dot{V}}
$$

Considering that $\bar{u}_{\text {mat }}$ must be always positive:

$$
\dot{\bar{U}}_{m a t}\left(\omega_{0}\right)<0 \rightarrow \dot{V}\left(\omega_{0}\right)<0 \rightarrow \operatorname{dir}\left(v_{m}\left(\omega_{0}\right)\right)=-\operatorname{dir}(d \boldsymbol{s})
$$

Considering the particular case of a passive material, $\overline{\boldsymbol{S}}$ must be zero during the non-equilibrium state, since the material cannot supply more power than the power it received from the EM field. As it is shown in [17], $\bar{U}_{\text {mat }}$ can be temporarily negative without violate causality if the total energy of the system $\left(\bar{U}_{\text {mat }}+\bar{U}_{f}\right)$ is positive. So, $\bar{U}_{\text {mat }}<0$ is a metastable state. Metastability denotes a temporary configuration of a dynamic system other than the state of least energy. The negative $\bar{U}_{\text {mat }}$ can be physically interpreted as the energy borrowed from the material by the EM field and that is given back to it when the system becomes stable again.

$$
\begin{gathered}
\bar{u}_{m a t}>\bar{u}_{f} \rightarrow \overline{\boldsymbol{S}}=0 \rightarrow v_{p}\left(\omega_{0}\right) \bar{u}_{f}\left(\omega_{0}\right)=v_{m}\left(\omega_{0}\right) \bar{u}_{\text {mat }}\left(\omega_{0}\right) \\
v_{p}\left(\omega_{0}\right)=v_{m}\left(\omega_{0}\right)\left(\frac{\bar{u}_{m a t}\left(\omega_{0}\right)}{\bar{u}_{f}\left(\omega_{0}\right)}\right)
\end{gathered}
$$




$$
\begin{aligned}
& \operatorname{dir}\left(v_{p}\left(\omega_{0}\right)\right)=\operatorname{dir}\left(v_{m}\left(\omega_{0}\right)\right) \rightarrow \operatorname{dir}\left(v_{p}\left(\omega_{0}\right)\right)=-\operatorname{dir}(\boldsymbol{s}) \\
& \text { since } \operatorname{dir}(\boldsymbol{k})=\operatorname{dir}(\boldsymbol{s}), \quad \text { then } \operatorname{dir}\left(v_{p}\left(\omega_{0}\right)\right)=-\operatorname{dir}(\boldsymbol{k})
\end{aligned}
$$

At first glance, a negative $v_{p}$ seems to indicate that energy is being inversely transported from the front side of the wave packet into its back side, which is senseless. Actually, it implies only that the direction of energy transportation has been reversed at this particular frequency, indicating that its correspondent mode, which is the resonance mode, is increasing in power.

Since the medium is passive (there is no other source beside the EM field), the "surplus power" borrowed from the medium by the resonance mode must come from the attenuation of the other modes of the EM field. Notice that $\overline{\boldsymbol{S}}$ is zero when the "apparent amplification" takes place, indicating that the process does not violate the law of energy conservation.

2.10.

\section{Electromagnetic coupling}

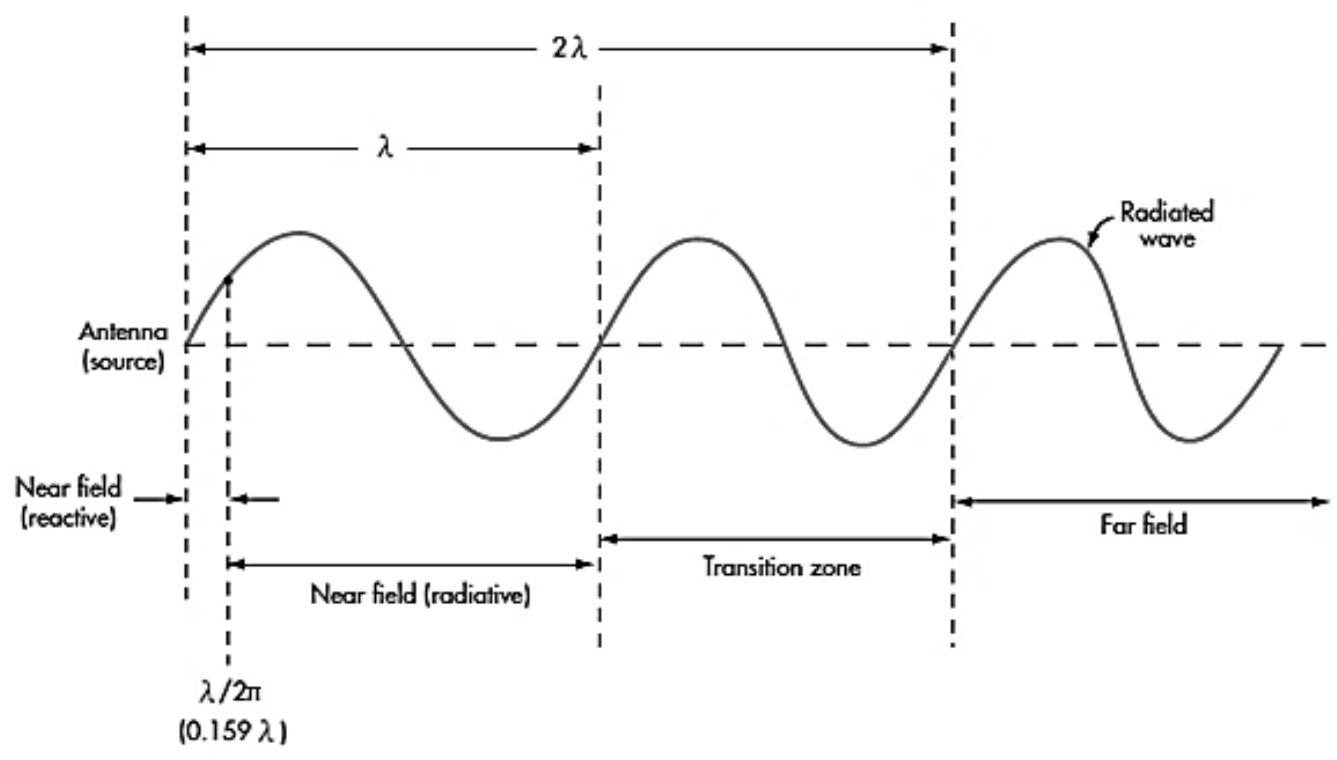

Figure 2.4: Electromagnetic-field zones as a function of the wavelength $\lambda$ 


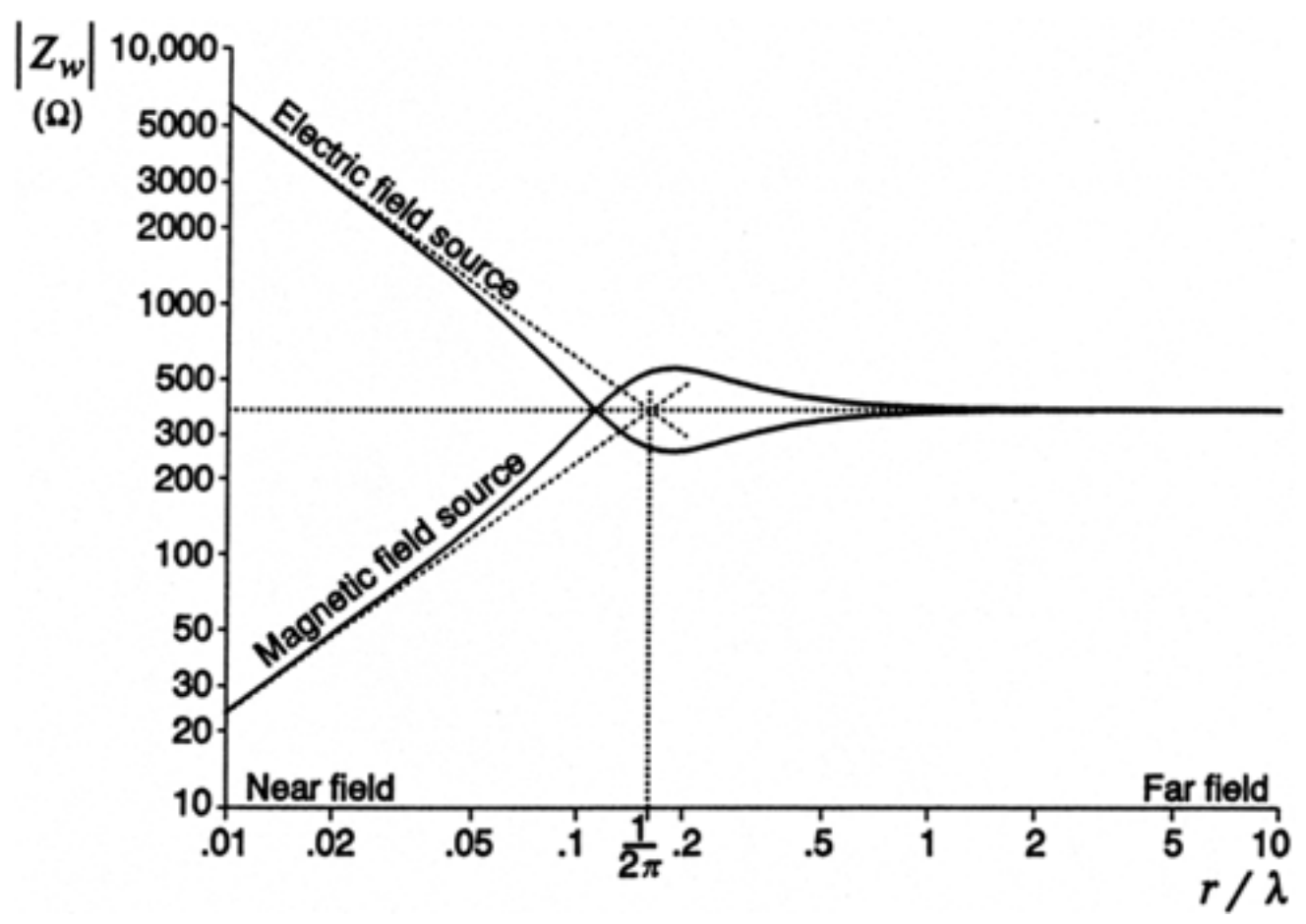

Figure 2.5: One of the fields is predominant in the near-field zone

An electrically small antenna (ESA) is one having overall dimensions (including the ground place) less than one-quarter wavelength $(\lambda / 4)$. It is characterized by presenting poor radiation efficiency and being very reactive (most of the power supplied to an ESA remains stored in the surrounding medium).

The space around an antenna is subdivided into three regions: the reactive near field, the Fresnel region and the far field (see Figure 2.4). For an ESA, this division can be reduced to two spatial zones only: the near field (quasistatic) and the far field (radiation).

The theoretical boundary between them is defined as sphere of radius $r$ :

$$
r=\frac{\lambda}{2 \pi}
$$

In the near field $\left(r \leq \frac{\lambda}{2 \pi}\right)$, one of the oscillating fields is predominant and remains stored in the medium either as magnetic potential $V_{m}$ or as 
electric one $V_{e}$ (see Figure 2.5). It can be represented as an evanescent EM wave (which is in essence the destructive sum of two propagating EM waves going in opposite directions). So, almost no energy is lost by the source of a quasistatic field because the EM waves cannot propagate away from the source (the EM modes that are generated by the source are quickly reabsorbed into it).

Nonetheless, when a proper receiver gets close enough of source, some EM modes in its near field can be "caught" by the receiver's near field, effectively transferring power from one circuit to the other. This phenomenon is called electromagnetic coupling.

Since the near field is spatially restricted, the EM coupling can only occur between two systems if they are separated by a distance $D \leq r$.

If two systems are coupled by means of a quasistatic electric field, the phenomenon is called electric induction. If they are coupled by means of a quasistatic magnetic field, it is called magnetic induction. 


\section{3}

\section{Generalized theory of transmission lines}

In this chapter, the concepts of virtual charges, currents and impedances are introduced in order to describe EM-coupled systems through transmission line formalism. The electro and magnetic-motive forces and their relationship with EM field propagation are discussed. The equations for electric and magnetic transmission lines (physical and virtual) are developed and the main results are presented.

\section{1.}

\section{Introduction: transmission line basic concepts}

Transmission line (TL) is one of the most discussed topics in electromagnetics. However, most of the present literature concentrates exclusively on electric transmission lines (ELTL) or the guidance of electromagnetic energy using two or multiple electric conductors (two-wire TL, coaxial cable, stripline and so on). Only recently a more general concept of the TL has been proposed aiming to include also magnetic transmission lines (MGTL) or the guidance of electromagnetic energy by means of magnetic conductors [18].

The MGTL proposed by [18], however, conceives MGTL as "electromagnetic energy guided by means of time-varying magnetic flux", which is not exactly the complementary of conventional ELTLs. In ELTLs, the EM fields are guided by currents made of moving electric charges (electric conduction currents). Thus, its true complementary is a hypothetical TL where the fields are steered by moving magnetic charges (magnetic conduction current).

Nonetheless, [18] has demonstrated that EM fields guided by a timevarying flux present TL-like behavior. Since electric and magnetic fluxes 
have the same dimension of electric and magnetic charges, respectively, a time-varying flux acts as a sort of "virtual current" driving the fields.

It can be easily shown that time-varying fluxes are currents:

$$
\begin{aligned}
j \omega \psi_{e}=j \omega \iint \boldsymbol{D} \cdot d \boldsymbol{s} & =\iint j \omega \boldsymbol{D} \cdot d \boldsymbol{s}=\iint \boldsymbol{J}_{e, d} \cdot d \boldsymbol{s}=I_{e, d} \\
j \omega \psi_{m} & =j \omega \iint \boldsymbol{B} \cdot d \boldsymbol{s}=\iint j \omega \boldsymbol{B} \cdot d \boldsymbol{s}=\iint \boldsymbol{J}_{m, d} \cdot d \boldsymbol{s}=I_{m, d}
\end{aligned}
$$

By taking the displacement currents as virtual currents, the fluxes as virtual charges and the reluctance to the flux of the medium as virtual impedances or admittances, the phenomena of electric and magnetic induction (electromagnetic coupling) can be effectively described by TL equations.

According to the Collins Dictionary [19], the word virtual means "so nearly true that for most purposes it can be regarded as true", "having the essence or effect but not the appearance or form of" or "being such practically or in effect, although not in actual fact or name". It is being applied to induction circuits because fluxes produce virtually the same effect of physical charges concerning guidance.

It is then defined as physical TLs the ones where EM fields are guided by means of physical currents (conduction currents) and as virtual $T L s$ the ones where EM fields are guided by means of virtual currents (or displacement currents).

The considered TLs are two-conductors TLs for which the incremental length of the line $d z$ causes a series potential loss $d V$ and shunt current $d I$. The TL theory postulates that the potential loss is proportional to the line current $\boldsymbol{I}[9]$ :

$$
d V=Z I d z
$$

where $Z$ is a series impedance per unit length.

And that the shunt current is proportional to the line potential: 


$$
d I=Y V d z
$$

where $Y$ is a shunt admittance per unit length.

Then, the propagation equations of a TL are given by:

$$
\begin{aligned}
& \frac{d V}{d z}=Z I \\
& \frac{d I}{d z}=Y V
\end{aligned}
$$

Taking the derivative of the equations and replacing them into the other, it can be shown that $V$ and $I$ are time-independent forms of the wave equation (Helmholtz equations):

$$
\begin{aligned}
& \frac{d^{2} V}{d z^{2}}-Z Y V=0 \\
& \frac{d^{2} I}{d z^{2}}-Z Y I=0
\end{aligned}
$$

The general solution of the TL equations is the sum of an incident wave $V_{i}$ and a reflected wave $V_{r}$ with propagation constant $\gamma$ :

$$
\begin{gathered}
V=V^{i}+V^{r}=V^{i}(0) e^{-\gamma z}+V^{r}(0) e^{\gamma z} \\
\gamma=\sqrt{Z Y} \\
\alpha=\operatorname{Re}\{\gamma\} \\
\beta=\operatorname{Im}\{\gamma\}
\end{gathered}
$$

where $\alpha$ is the attenuation factor and $\beta$ is the propagation constant of the line.

By definition, the load impedance $Z_{L}$ is at $z=0$. The characteristic impedance $Z_{0}$ is the total impedance added by the TL itself and the input impedance $Z_{\text {in }}$ is the total impedance seen from the source. 


\section{2.}

\section{Electro-motive and magneto-motive forces}

Electro-motive force (EMF) was first defined as the electrical energy per unit of electric charge supplied by the source of a physical ELTL:

$$
E M F=\frac{\partial U_{e}}{\partial q_{e}}
$$

The sources of EMF can be the electrical field itself (once electrical energy is the energy stored in the form of an electric field) or any device that converts any other form of energy into the electrical one, such as a battery (chemical to electrical) or a dynamo (mechanical to electrical). The word "force", in this case, does not mean a mechanical force, measured in newtons, but an electric potential, measured in volts:

$$
E M F=V_{e}(b)-V_{e}(a)=\int_{b}^{a} \boldsymbol{E} \cdot d \boldsymbol{l}[V]
$$

Historically speaking, the name EMF was attribute to $V_{e}$ based on the misconception of the $18^{\text {th }}$ century that electrical energy was transported by the electric charges all the way along the intervening space between two separated points [20]. EMF was then a scalar quantity that indicated the necessary force to move a charge from point $a$ to $b$ or the force produced when two points in different potentials were connected via an electric conductor:

$$
E M F=\frac{|\boldsymbol{F}|}{q_{e}}
$$

After the publication of Faraday's work, it was empirically demonstrated that the so-called "electrical energy" could be sent from one place to the other using an intervening time-varying magnetic flux 
generated by a current loop in replacement of the physical electric charges, forming a magnetic induction circuit or a virtual magnetic $T L$ (VMGTL). According to Faraday-Lenz law of induction:

$$
E M F=-j \omega \psi_{m}
$$

Based on previous definitions, it was also called EMF or electric potential. However, it is misleading since the EMF of a VMGTL (differently of the ELTL case) is not a real potential. Real potentials are defined over path-independent conservative fields and the EMF of a VMGTL is a circulation (hence, a non-conservative one):

$$
\begin{gathered}
E M F=-j \omega \psi_{m}=-j \omega \iint \boldsymbol{B} \cdot d \boldsymbol{s}=-\iint j \omega \boldsymbol{B} \cdot d \boldsymbol{s} \\
=-\iint \nabla \times \boldsymbol{E} \cdot d \boldsymbol{s}=\oint \boldsymbol{E} \cdot d \boldsymbol{l}
\end{gathered}
$$

In other words, it is not a state function. For a fixed $\psi_{m}$, with $\psi_{m} \neq 0$ :

$$
\begin{gathered}
\lim _{\omega \rightarrow \infty}(|E M F|)=\lim _{\omega \rightarrow \infty}\left(\left|\omega \psi_{m}\right|\right)=\infty \\
\lim _{\omega \rightarrow 0}(|E M F|)=\lim _{\omega \rightarrow 0}\left(\left|\omega \psi_{m}\right|\right)=0
\end{gathered}
$$

Analogously, for a fixed $\omega$, with $\omega \neq 0$ :

$$
\begin{gathered}
\lim _{\psi_{m} \rightarrow \infty}(|E M F|)=\lim _{\psi_{m} \rightarrow \infty}\left(\left|\omega \psi_{m}\right|\right)=\infty \\
\lim _{\psi_{m} \rightarrow 0}(|E M F|)=\lim _{\psi_{m} \rightarrow 0}\left(\left|\omega \psi_{m}\right|\right)=0
\end{gathered}
$$

which implies that it can be made arbitrarily great or small independently of the configuration of the system.

For this reason, the EMF of a VMGTL is better described as a virtual potential (a current source). 
Since the intensity of $\psi_{m}$ depends on both $\boldsymbol{B}$ and the effective area it is going through, the total virtual EMF obtained from a fixed $\boldsymbol{B}$ can vary a lot depend on how well channeled the flux is. This is the case, for example, of the conventional electric transformer, the most famous VMGTL presented in the literature. In general, it employs a ferromagnetic material $\left(\mu_{r} \gg 1\right)$ to limit $\psi_{m}$ to a fixed path $l$ and transversal section $A$.

In order to clarify this particular point, it is interesting to give a look at the Poynting vector in an electric transformer.

In the electric transformer's problem, the magneto-motive force (MMF) is usually defined as the magnetic flux $\psi_{m}$ times the magnetic reluctance $\mathcal{R}_{m}$ (the opposition of the medium to the magnetic flux), analogously to the Ohm's law for ELTLs:

$$
M M F=\psi_{m} \mathcal{R}_{m}
$$

The total MMF of an electric transform is real because fluxes going in opposite directions possess inverted magnetic potential. As a consequence, there must be a uniform magnetic field stored between them, according to:

$$
M M F=\psi_{m} \mathcal{R}_{m}=V_{m}=\int_{a}^{b} \boldsymbol{H} \cdot d \boldsymbol{l}[A]
$$

which demonstrates that the conservative field of the system is the magnetic field stored between the incoming and outgoing magnetic flux.

Power flow in a VMGTL is the product of this magnetic field stored between the fluxes and the electric field generated by the time-varying flux in the form of electric-field circulation [21] (see Figure 3.1).

$$
P=j \omega \psi_{m} V_{m}=(E M F)(M M F)
$$




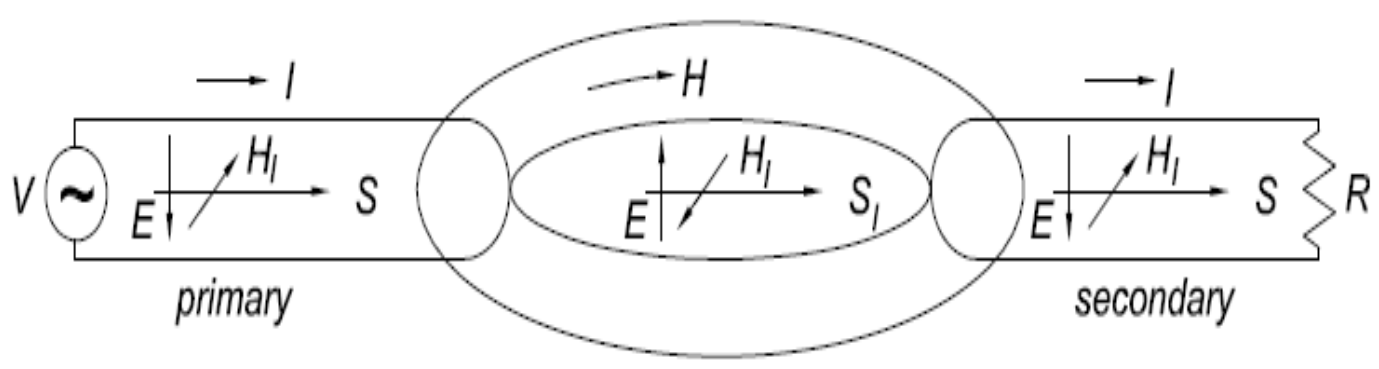

Figure 3.1: The difference of magnetic potential between the incoming and ongoing fluxes causes a transversal magnetic field component to be stored between them. It is this component that is responsible for power flow, not the magnetic field paralell to the flux

When the incoming and ongoing fluxes are spatially distant from each other and confined in well-defined paths, the obtained $M M F$ is much greater, and that is why power transfer is improved. Since $\mathcal{R}_{m}$ is a characteristic of the medium, it is $\psi_{m}$ that increases when the MMF is augmented, and the only way to increase $\psi_{m}$ without supplying additional power to the source loop is to confine $\psi_{m}$.

In the same manner, it could be said that an electric induction circuit or virtual electric TL (VELTL) possesses a virtual MMF:

$$
M M F=j \omega \psi_{e}
$$

And a real EMF between the incoming and outgoing electric flux:

$$
E M F=\psi_{e} \mathcal{R}_{e}=V_{e}=\int_{a}^{b} \boldsymbol{E} \cdot d \boldsymbol{l}
$$

where $\mathcal{R}_{e}$ is the electric reluctance.

As in VMGTLs, power flow is also the product of a real potential with a virtual one.

Now, let's consider again the classical ELTL. It can be easily shown it possesses also a virtual potential: 


$$
\begin{aligned}
M M F=V_{m}= & \oint \boldsymbol{H} \cdot d \boldsymbol{l}=\iint \nabla \times \boldsymbol{H} \cdot d \boldsymbol{s}=\iint \boldsymbol{J}_{e} \cdot d \boldsymbol{s} \\
& =\iint\left(\sigma_{e} \boldsymbol{E}+j \omega \boldsymbol{D}\right) \cdot d \boldsymbol{s}=\iint \sigma_{e} \boldsymbol{E} \cdot d \boldsymbol{s}+\iint j \omega \boldsymbol{D} \cdot d \boldsymbol{s} \\
& =I_{e, c}+j \omega \psi_{e}=I_{e}
\end{aligned}
$$

As it was previously discussed, virtual potentials do not store energy. The term $j \omega \psi_{e}$ is a field circulation while $I_{e, c}$ is the dissipative conduction current, hence both non-conservative.

As it is well-known, power flow in ELTLs is equal to the product of the electric field stored between the electrical conductors and the magneticfield circulation generated by the electric current.

$$
P=(E M F)(M M F)=V_{e} I_{e}
$$

So, the EMF is the only real potential in an ELTL.

Now, let's consider a physical MGTL. The existence of magnetic charges $q_{m}$ and wires made of highly magnetic conductive materials $\left(\sigma_{m} \gg 1\right)$ are assumed. In this case, the MMF can also be defined as the magnetic energy per unit of magnetic charge supplied by the source in the magnetic circuit:

$$
M M F=\frac{\partial U_{m}}{\partial q_{m}} \equiv V_{m}=\int \boldsymbol{H} \cdot d \boldsymbol{l}
$$

And analogously to the electric conductive counterpart:

$$
\begin{aligned}
E M F=\oint \boldsymbol{E} \cdot d \boldsymbol{l} & =-\iint \nabla \times \boldsymbol{E} \cdot d \boldsymbol{s}=-\iint \boldsymbol{J}_{m} \cdot d \boldsymbol{s} \\
= & -\iint\left(\sigma_{m} \boldsymbol{H}+j \omega \boldsymbol{B}\right) \cdot d \boldsymbol{s} \\
= & -\iint \sigma_{m} \boldsymbol{H} \cdot d \boldsymbol{s}-\iint j \omega \boldsymbol{B} \cdot d \boldsymbol{s}=-I_{m, c}-j \omega \psi_{m}
\end{aligned}
$$



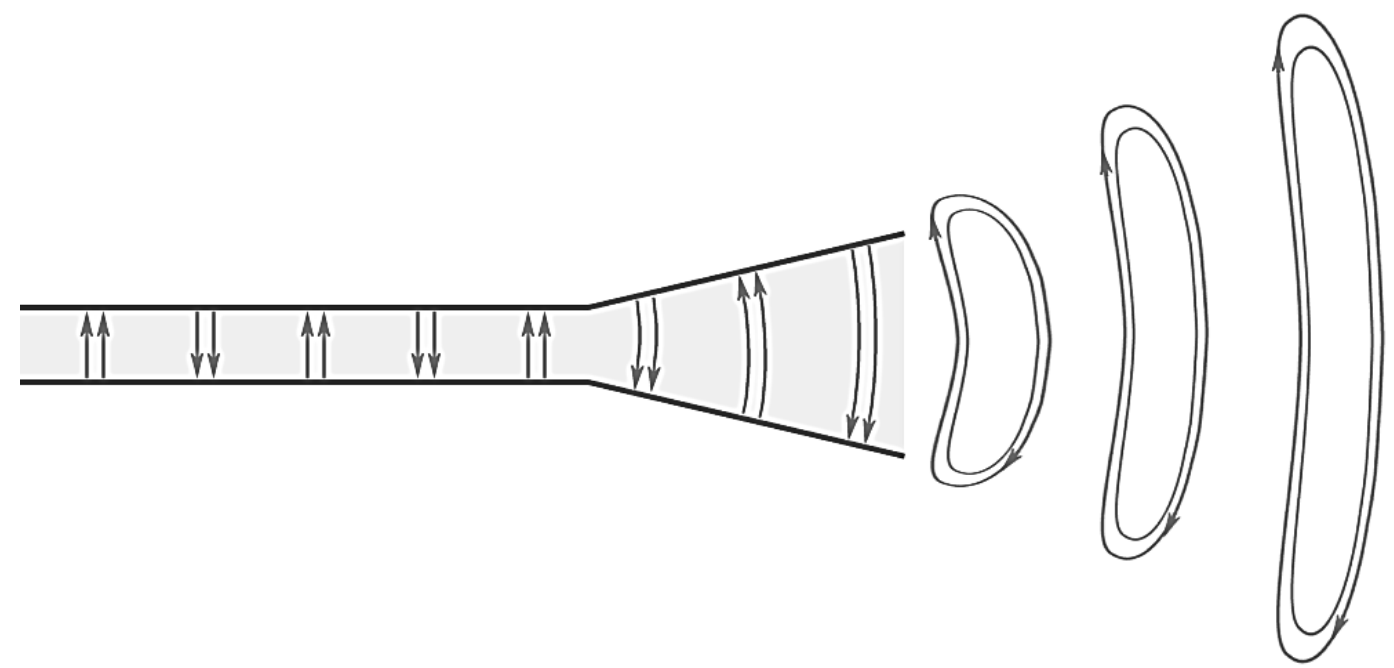

Figure 3.2: For guided modes, one of the fields is the source of a real potential. For radiated modes, both fields are circulations (hence, virtual potentials)

Hence, like in virtual TLs, power flow is also the product of a virtual and a real potential in physical TLs (ELTL and MGTL). The only difference between ELTL and MGTL is that in a MGTL it is the MMF that is the real one.

The proposed concept can even be extent for a more general case in order to classify in terms of EMF and MMF the non-guided propagation as well.

When the EM fields are said to be "radiated", both electric and magnetic fields become circulations [8] [9] (see Figure 3.2). The amount of power that is radiated by the TL depends on a parameter called radiation resistance, which is defined as "a virtual resistance that does not physically exist but is a quantity coupling the antenna to a distant region in space via a virtual transmission line" [22]. This has nothing to do with the thermal state of the antenna itself but is related with the temperature of distant objects that the antenna is looking at.

Considering that at least one real potential is need to confine the EM waves in a specific region, it can be said that the real motive force of the circuit is converted into a virtual one whenever the fields start to leave the $\mathrm{TL}$ in the form of radiation. Thus, the radiation resistance of an antenna could be interpreted as a measure of efficiency in this conversion process. 
An observation must be pointed out here. Once the fields are radiated, no energy is conserved in a determined region of the space due to the non-conservative fields that cause spontaneous phase shift in the wave front. According to Atkins [23], "spontaneous changes are always accompanied by a dispersal of energy". So, the spontaneous phase shift causes spatial power dispersion in the radiated EM field as a consequence of the entropy generation process. In all cases, the source of this entropy generation is the virtual potentials (currents).

For confined EM waves (in real or virtual TLs), the virtual potential is controlled by a real one, allowing the energy dispersion due to wave propagation to be minimized (phase shift is not spontaneous anymore since there is a real and well-defined potential generating the currents).

One could ask what would happen if both motive forces were real. This is the case of a standing wave. Considering that at least one virtual motive force is needed for wave propagation, two real potentials results in no propagation at all.

\begin{tabular}{|c|c|c|}
\hline & EMF & MMF \\
\hline ELTL & Real & Virtual \\
\hline MGTL & Virtual & Real \\
\hline VELTL & Real & Virtual \\
\hline VMGTL & Virtual & Real \\
\hline EM radiation & Virtual & Virtual \\
\hline Standing wave & Real & Real \\
\hline
\end{tabular}

Table 10: Classification of transmission lines in terms of electric and magnetic potentials

From Table 10 comes the conclusion that virtual potentials are responsible for the displacement and power dissipation of the EM waves while real potentials are responsible for their confinement in a specific path (guidance).

So, a TL is said to be electric if it has a real electric potential and it is said to be magnetic if it has a real magnetic potential. 


\section{3.}

\section{Physical transmission lines}

\subsection{1.}

\section{Electric transmission line}

The two-wire electric transmission line (ELTL) is defined as two parallel electric conductors of length $l$ insulated from each other by a nonelectric conductive medium terminated on a linear load fed by a timeharmonic EMF (see Figure 3.3). It is characterized by its electric conductivity $\sigma_{e}$ and the electromagnetic properties of the surrounding medium. The insulator medium of the line is assumed to be imperfect $\left(\varepsilon^{\prime \prime}>0, \mu^{\prime \prime}>0\right)$ as well as the electric conductors $\left(\sigma_{e}<\infty\right)$.

The finite conductivity of the electric wires results in a longitudinal component of the electric field $E_{z}$, which is responsible for the power dissipation at the level of the conductor.

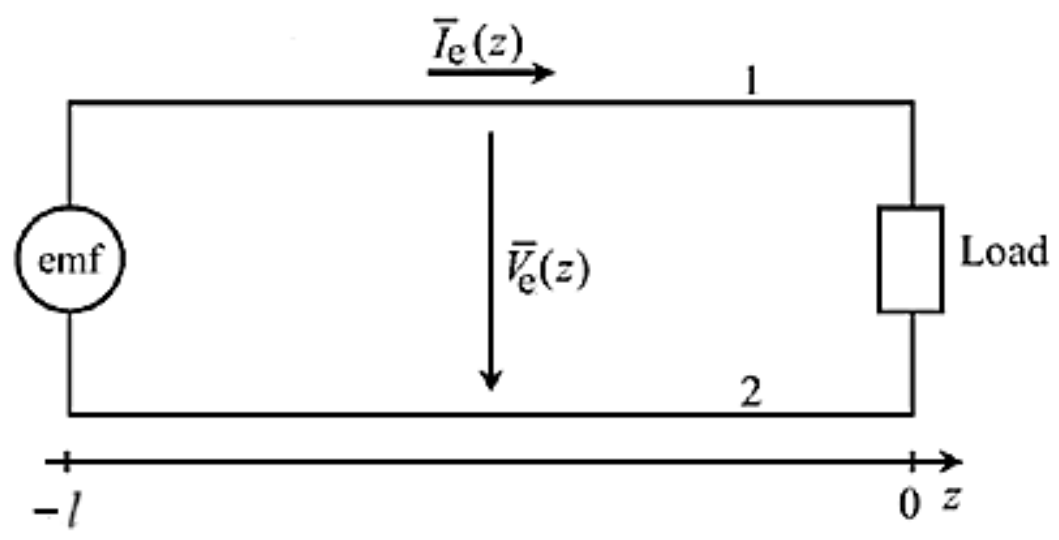

Figure 3.3: Schematic of an electric transmission line

The electric potential $V_{e}$ and the electric conduction current $I_{e, c}$ of the ELTL are waves that satisfy the following propagation equations:

$$
\begin{gathered}
\frac{d}{d z} V_{e}(z)=\left(R_{e}^{\prime}+G_{m}^{\prime}+j \omega L^{\prime}\right) I_{e, c}(z)=Z_{e} I_{e, c}(z) \\
\frac{d}{d z} I_{e, c}(z)=\left(G_{e}^{\prime}+j \omega C^{\prime}\right) V_{e}(z)=Y_{e} V_{e}(z)
\end{gathered}
$$


where $Z_{e}, Y_{e}$ and $Z_{e, 0}$ are the electrical series impedance, the shunt admittance and the characteristic impedance of the ELTL, $g_{R}^{\prime}$ is a dimensionless geometric function, and terms $R_{e}^{\prime}, G_{e}^{\prime}, G_{m}^{\prime}, L^{\prime}$ and $C^{\prime}$ denote, respectively, the electrical resistance, the electrical conductance, the magnetic conductance, the inductance and the capacitance per unit length:

$$
\begin{gathered}
R_{e}^{\prime} \triangleq \frac{1}{\sigma_{e}} g_{R}^{\prime} \\
G_{e}^{\prime} \triangleq \omega C^{\prime} \frac{\varepsilon^{\prime \prime}}{\varepsilon^{\prime}}=\omega C^{\prime} \delta_{e} \\
G_{m}^{\prime} \triangleq \omega L^{\prime} \frac{\mu^{\prime \prime}}{\mu^{\prime}}=\omega L^{\prime} \delta_{m} \\
I_{e, c}(z)=j \omega q_{e} \\
Z_{e, 0} \triangleq \frac{V_{e}(z)}{I_{e, c}(z)}=\sqrt{\frac{Z_{e}}{Y_{e}}}=\sqrt{\frac{R_{e}^{\prime}+G_{m}^{\prime}+j \omega L^{\prime}}{G_{e}^{\prime}+j \omega C^{\prime}}}
\end{gathered}
$$

The complex propagation constant is given by:

$$
\gamma_{e}=\sqrt{Z_{e} Y_{e}}=\sqrt{\left(\frac{R_{e}^{\prime}}{j \omega}+\frac{G_{m}^{\prime}}{j \omega}+L^{\prime}\right)\left(\frac{G_{e}^{\prime}}{j \omega}+C^{\prime}\right)}
$$

\subsection{2.}

\section{Magnetic transmission line}

The two-wire magnetic transmission line (MGTL) is defined as two parallel magnetic conductors of length $l$ insulated from each other by a nonmagnetic conductive medium terminated on a linear load fed by a timeharmonic MMF (see Figure 3.4). It is characterized by its magnetic conductivity $\sigma_{m}$ and the electromagnetic properties of the surrounding medium. As before, the insulating medium and the magnetic conductors are supposed to be imperfect $\left(\varepsilon^{\prime \prime}>0, \mu^{\prime \prime}>0, \sigma_{m}<\infty\right)$. 


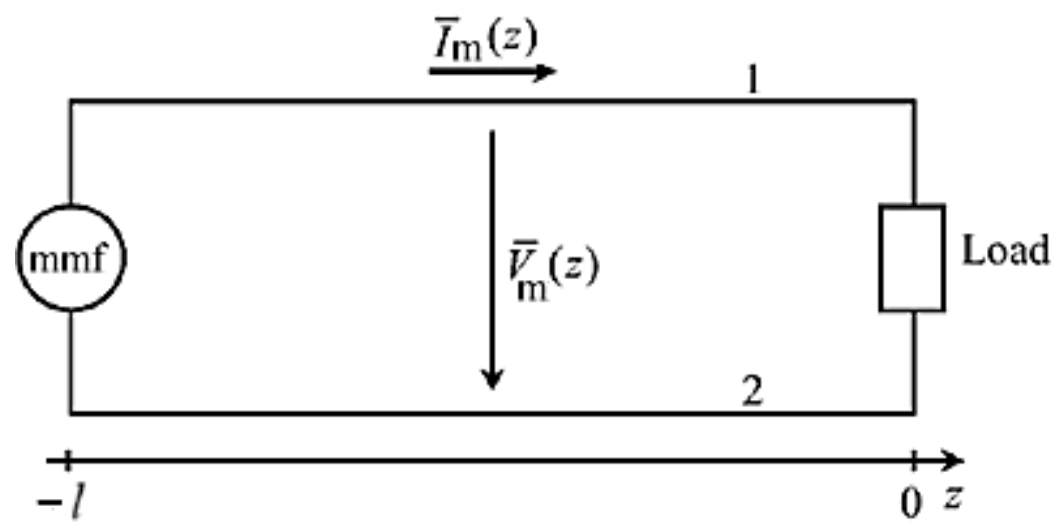

Figure 3.4: Schematic of a magnetic transmission line

Due to the symmetry of the Maxwell equations, the magnetic potential $V_{m}$ and magnetic conduction current $I_{m, c}$ of the MGTL are also waves that satisfy a set of propagation equations analogous to their electric counterpart:

$$
\begin{gathered}
\frac{d}{d z} V_{m}(z)=\left(R_{m}^{\prime}+G_{e}^{\prime}+j \omega C^{\prime}\right) I_{m, c}(z)=Y_{m} I_{m, c}(z) \\
\frac{d}{d z} I_{m, c}(z)=\left(G_{m}^{\prime}+j \omega L^{\prime}\right) V_{m}(z)=Z_{m} V_{m}(z)
\end{gathered}
$$

where $Z_{m}, Y_{m}$ and $Z_{m, 0}$ are the series impedance, the shunt admittance and the characteristic impedance. The terms $R_{m}^{\prime}$ and $G_{m}^{\prime}$ denote, respectively, the magnetic resistance and the magnetic conductance per unit length of the MGTL. They are given by:

$$
\begin{gathered}
R_{m}^{\prime} \triangleq \frac{1}{\sigma_{m}} g_{R}^{\prime} \\
I_{m, c}(z)=j \omega q_{m} \\
Z_{m, 0} \triangleq \frac{I_{m, c}(z)}{V_{m}(z)}=\sqrt{\frac{Z_{m}}{Y_{m}}}=\sqrt{\frac{G_{m}^{\prime}+j \omega L^{\prime}}{R_{m}^{\prime}+G_{e}^{\prime}+j \omega C^{\prime}}}
\end{gathered}
$$

The complex propagation constant is given by: 


$$
\gamma_{m}=\sqrt{Z_{m} Y_{m}}=\sqrt{\left(\frac{G_{m}^{\prime}}{j \omega}+L^{\prime}\right)\left(\frac{R_{m}^{\prime}}{j \omega}+\frac{G_{e}^{\prime}}{j \omega}+C^{\prime}\right)}
$$

\section{4.}

\section{Virtual transmission lines}

\subsection{1.}

\section{Magnetic transmission line}

The virtual magnetic transmission line (VMGTL) is defined as a magnetic circuit formed between two insulated drivers (no physical charge is flowing from one driver to the other) coupled by an intervening quasistatic magnetic field (see Figure 3.5 and Figure 3.6). The transmitting driver is the MMF source generating the time-varying magnetic flux (magnetic displacement current) that links the terminals. The receiving driver is the transducer connected to the load that converts the virtual magnetic charges (the magnetic flux) to physical ones. A virtual MGTL is characterized only by the EM properties of the medium penetrated by the magnetic flux.

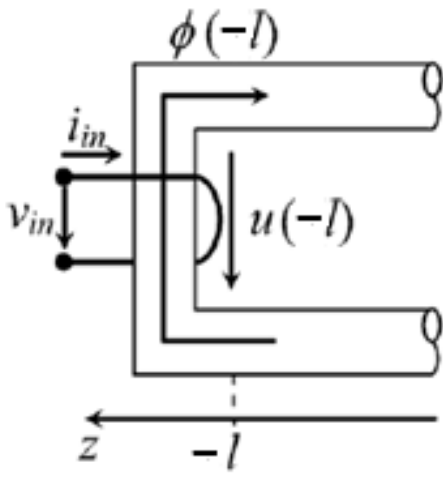

(a)

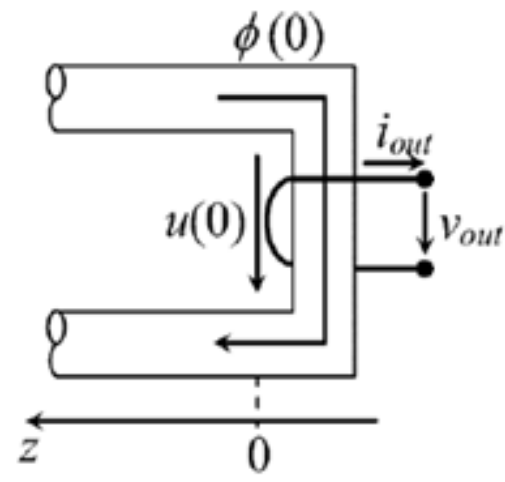

(b)

Figure 3.5: Terminals of a virtual magnetic transmission line 


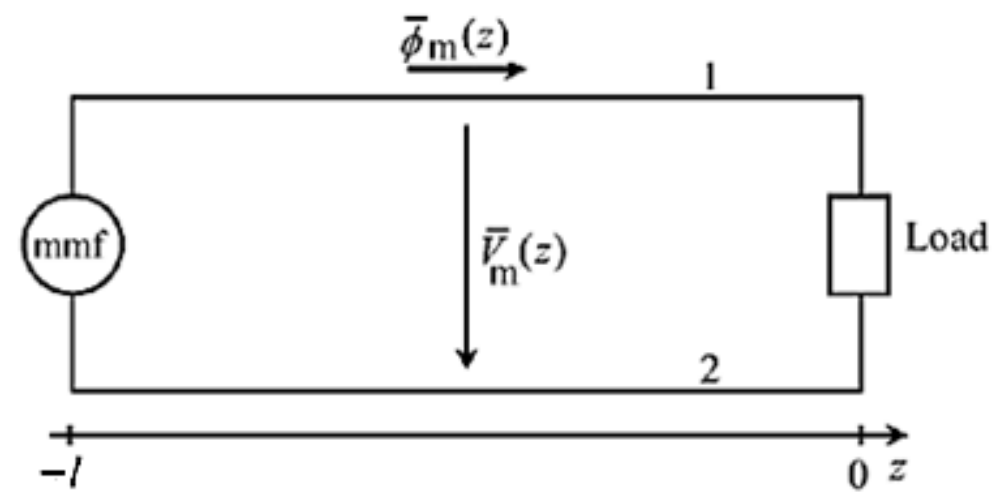

Figure 3.6: Schematic of a virtual magnetic transmission line

As the physical TLs, the virtual TLs obey correspondent propagation equations in terms of their potential and virtual currents. For VMGTLs, the magnetic potential $V_{m, v}$ and the magnetic displacement current $I_{m, d}$ obey the following propagation equations:

$$
\begin{gathered}
\frac{d}{d z} V_{m, v}(z)=\left(\frac{\mathcal{R}_{m}^{\prime}}{j \omega}+G_{e}^{\prime}+j \omega C^{\prime}\right) I_{m, d}(z)=Y_{m, v} I_{m, d}(z) \\
\frac{d}{d z} I_{m, d}(z)=\left(G_{m}^{\prime}+j \omega L^{\prime}\right) V_{m, v}(z)=Z_{m, v} V_{m, v}(z)
\end{gathered}
$$

where $Z_{m, v}, Y_{m, v}$ and $\mathcal{R}_{m}^{\prime}$ are the series impedance, the shunt admittance, and the magnetic reluctance per unit length, respectively.

The virtual magnetic current $I_{m, d}$ and the characteristic impedance $Z_{m, v, 0}$ of the VMGTL are given by:

$$
\begin{gathered}
I_{m, d}(z)=j \omega \psi_{m}(z) \\
Z_{m, v, 0}=\frac{I_{m, d}(z)}{V_{m, v}(z)}=\sqrt{\frac{Z_{m, v}}{Y_{m, v}}}=\sqrt{\frac{G_{m}^{\prime}+j \omega L^{\prime}}{\frac{\mathcal{R}_{m}^{\prime}}{j \omega}+G_{e}^{\prime}+j \omega C^{\prime}}}
\end{gathered}
$$

The term $\frac{\mathcal{R}_{m}^{\prime}}{j \omega}$ can be said to be a virtual admittance because it neither stores nor dissipate any real power. Like the radiation resistance, it has no 
relationship with the thermal equilibrium of the antenna that is generating the flux.

The virtual potential and the virtual current can be identified and converted into the physical potentials and the physical currents in the terminals through:

$$
\begin{aligned}
& V_{m, v}(-l)=V_{m, v}^{\text {in }}=I_{e, c}^{\text {in }} \\
& I_{m, d}(-l)=I_{m, d}^{\text {in }}=V_{e, c}^{\text {in }} \\
& V_{m, v}(0)=V_{m, v}^{\text {out }}=I_{e, c}^{\text {out }} \\
& I_{m, d}(0)=I_{m, d}^{\text {out }}=V_{e, c}^{\text {out }}
\end{aligned}
$$

Making the simplifying hypothesis that the magnetic flux is mostly confined in a circuit with circular transversal region of fixed radius, the real and the imaginary parts of $\mathcal{R}_{m}^{\prime}$ can be approximated by [18]:

$$
\begin{gathered}
\operatorname{Re}\left\{\mathcal{R}_{m}^{\prime}\right\}=2 \frac{\left(\bar{U}_{m}-\bar{U}_{e}\right)}{\psi_{m}^{2}} \\
\operatorname{Im}\left\{\mathcal{R}_{m}^{\prime}\right\}=\frac{P_{d, d}}{\omega \psi_{m}^{2}}
\end{gathered}
$$

where $P_{d, d}$ is the total power dissipation due to material losses.

Notice that the real part of $\mathcal{R}_{m}$ can assume either positive or negative values depending on the balance of electric and magnetic energy storage in the circuit, while, as expected, the imaginary part of $\mathcal{R}_{m}$ is always positive (once it accounts for the losses).

The complex propagation constant is given by:

$$
\gamma_{m, v}=\sqrt{Z_{m, v} Y_{m, v}}=\sqrt{\left(\frac{G_{m}^{\prime}}{j \omega}+L^{\prime}\right)\left(-\frac{\mathcal{R}_{m}^{\prime}}{\omega^{2}}+\frac{G_{e}^{\prime}}{j \omega}+C^{\prime}\right)}
$$




\subsection{2.}

\section{Electric transmission line}

The virtual electric transmission line (VELTL) is defined as an electric circuit formed between two galvanic insulated drivers (no physical charge is flowing from one driver to the other) coupled by an intervening quasistatic electric field (see Figure 3.7). The transmitting driver is the EMF source generating the time-varying electric flux (electric displacement current) that links the terminals and the receiving driver is the transducer connected to the load that converts the virtual electric charges (the electric flux) to physical ones. Like the previous case, a virtual ELTL is characterized only by the EM properties of the medium penetrated by the flux.

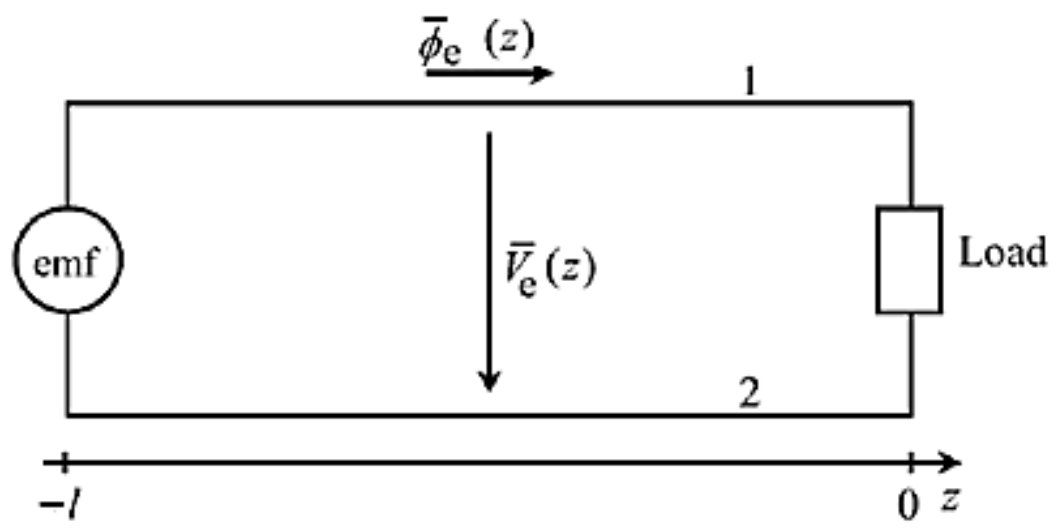

Figure 3.7: Schematic of a virtual electric transmission line

The VELTL is symmetric of the VMGTL, so its propagation equations are given by:

$$
\begin{gathered}
\frac{d}{d z} V_{e, v}(z)=\left(\frac{\mathcal{R}_{e}^{\prime}}{j \omega}+G_{m}^{\prime}+j \omega L^{\prime}\right) I_{e, d}(z)=Z_{e, v}(z) I_{e, d}(z) \\
\frac{d}{d z} I_{e, d}(z)=\left(G_{e}^{\prime}+j \omega C^{\prime}\right) V_{e, v}(z)=Y_{e, v}(z) V_{e, v}(z)
\end{gathered}
$$

where $Z_{e, v}, Y_{e, v}$ and $\mathcal{R}_{e}^{\prime}$ are the series impedance, the shunt admittance, and the electric reluctance per unit length, respectively. 
The virtual electric current $I_{e, d}$ and the characteristic impedance $Z_{e, v, 0}$ of the VELTL are given by:

$$
\begin{gathered}
I_{e, d}(z)=j \omega \psi_{e}(z) \\
Z_{e, v, 0}=\frac{V_{e, v}(z)}{I_{e, d}(z)}=\sqrt{\frac{Z_{e, v}}{Y_{e, v}}}=\sqrt{\frac{\frac{\mathcal{R}_{e}^{\prime}}{j \omega}+G_{m}^{\prime}+j \omega L^{\prime}}{G_{e}^{\prime}+j \omega C^{\prime}}}
\end{gathered}
$$

Analogously to its magnetic counterpart, $\frac{\mathcal{R}_{e}^{\prime}}{j \omega}$ is a virtual impedance.

The virtual potential and the virtual current can be identified and converted into the physical potentials and the physical currents in the terminals through:

$$
\begin{gathered}
V_{e, v}(-l)=V_{e, v}^{i n}=V_{e, c}^{i n} \\
I_{e, d}(-l)=I_{e, d}^{i n}=I_{e, c}^{i, c} \\
V_{e, v}(0)=V_{e, v}^{o u t}=V_{e, c}^{o u t} \\
I_{e, d}(0)=I_{e, d}^{o u t}=I_{e, c}^{o u t}
\end{gathered}
$$

Doing the same simplifying hypothesis that the electric flux is mostly confined in well-determined cylindrical transversal region of fixed radius, the real and the imaginary parts of $\mathcal{R}_{e}$ can be approximated by:

$$
\begin{gathered}
\operatorname{Re}\left\{\mathcal{R}_{e}^{\prime}\right\}=2 \frac{\left(\bar{U}_{e}-\bar{U}_{m}\right)}{\psi_{e}^{2}} \\
\operatorname{Im}\left\{\mathcal{R}_{e}^{\prime}\right\}=\frac{P_{d, d}}{\omega \psi_{e}^{2}}
\end{gathered}
$$

The complex propagation constant is given by: 


$$
\gamma_{e, v}=\sqrt{Z_{e, v} Y_{e, v}}=\sqrt{\left(-\frac{\mathcal{R}_{e}^{\prime}}{\omega^{2}}+\frac{G_{m}^{\prime}}{j \omega}+L^{\prime}\right)\left(\frac{G_{e}^{\prime}}{j \omega}+C^{\prime}\right)}
$$

3.5 .

Solution of the propagation equations and time-average powers

\begin{tabular}{|c|c|c|c|c|}
\hline \multirow{2}{*}{$\begin{array}{c}\text { Physical } \\
\text { TL }\end{array}$} & Electric & $q_{e}$ and $I_{e, c}$ & $\sqrt{\frac{R_{e}^{\prime}+G_{m}^{\prime}+j \omega L^{\prime}}{G_{e}^{\prime}+j \omega C^{\prime}}}$ & $\sqrt{\left(\frac{R_{e}^{\prime}}{j \omega}+\frac{G_{m}^{\prime}}{j \omega}+L^{\prime}\right)\left(\frac{G_{e}^{\prime}}{j \omega}+C^{\prime}\right)}$ \\
\cline { 2 - 5 } & Magnetic & $q_{m}$ and $I_{m, c}$ & $\sqrt{\frac{G_{m}^{\prime}+j \omega L^{\prime}}{R_{m}^{\prime}+G_{e}^{\prime}+j \omega C^{\prime}}}$ & $\sqrt{\left(\frac{G_{m}^{\prime}}{j \omega}+L^{\prime}\right)\left(\frac{R_{m}^{\prime}}{j \omega}+\frac{G_{e}^{\prime}}{j \omega}+C^{\prime}\right)}$ \\
\hline \multirow{2}{*}{$\begin{array}{c}\text { Virtual } \\
\text { TL }\end{array}$} & Electric & $\psi_{e}$ and $I_{e, d}$ & $\sqrt{\frac{\mathcal{R}_{e}^{\prime}}{\frac{j \omega}{G_{e}^{\prime}+j \omega C_{m}^{\prime}+j \omega L^{\prime}}}}$ & $\sqrt{\left(-\frac{\mathcal{R}_{e}^{\prime}}{\omega^{2}}+\frac{G_{m}^{\prime}}{j \omega}+L^{\prime}\right)\left(\frac{G_{e}^{\prime}}{j \omega}+C^{\prime}\right)}$ \\
\cline { 2 - 5 } & Magnetic & $\psi_{m}$ and $I_{m, d}$ & $\sqrt{\frac{G_{m}^{\prime}+j \omega L^{\prime}}{j \omega}+G_{e}^{\prime}+j \omega C^{\prime}}$ & $\sqrt{\left(\frac{G_{m}^{\prime}}{j \omega}+L^{\prime}\right)\left(-\frac{\mathcal{R}_{m}^{\prime}}{\omega^{2}}+\frac{G_{e}^{\prime}}{j \omega}+C^{\prime}\right)}$ \\
\hline
\end{tabular}

Table 11: General transmission line characteristic impedances and propagation constants

The main results of the generalized TL equations are summarized in Table 11.

Physical and virtual TLs tend to the same characteristic impedance $Z_{0}$ and propagation constant $\gamma$ when their physical and virtual resistances are minimized:

$$
\begin{gathered}
Z_{0} \rightarrow \sqrt{\frac{G_{m}^{\prime}+j \omega L^{\prime}}{G_{e}^{\prime}+j \omega C^{\prime}}} \\
\gamma \rightarrow \sqrt{\left(\frac{G_{m}^{\prime}}{j \omega}+L^{\prime}\right)\left(\frac{G_{e}^{\prime}}{j \omega}+C^{\prime}\right)}
\end{gathered}
$$

If the fluxes in the VELTL and VMGTL are well channeled in a circuit of high permittivity and high permeability, respectively, with total length 
$l$ and transversal section $A$, their total reluctances along the virtual line tend approximately to:

$$
\begin{aligned}
\mathcal{R}_{m} & \rightarrow \frac{\ell}{\mu A}\left[H^{-1}\right] \\
\mathcal{R}_{e} & \rightarrow \frac{\ell}{\varepsilon A}\left[F^{-1}\right]
\end{aligned}
$$

This is the case, for example, of classical electrical transformers, in which ferromagnetic materials $\left(\mu_{r} \gg 1\right)$ are used to create a magnetic path with a very small $\mathcal{R}_{m}$. In the present perspective that circuits coupled via electric or magnetic induction can be understood as some sort of $T L$, it becomes evident that when the module of $\mathcal{R}_{m}$ becomes negligible in comparison with the other terms, the impedance of the VMGTL perfectly matches with the impedance of the primary and secondary circuits (which are conventional ELTLs), and since they have almost the same impedance the EM wave can flow from the ELTL of the primary to the VMGTL, and from the VMGTL to the ELTL of the secondary, with almost no reflection, and that is the reason why the efficiency of the transformer is enhanced.

By evaluating the Poynting vector in a transformer, it is clear that power is flowing in the intervening space between the fluxes (analogously to the ELTL case, where power flows between the electric wires). And both cases, physical and virtual TLs, the currents or fluxes are only guiding the EM wave, but they are not conveying real power. The conduction current in the ELTL is destroying power by dissipating it in the form of heat while the displacement current in the VMGTL generates only a virtual potential (which is not conservative, as discussed in Section 3.2).

Considering that the presented TLs - physical and virtual - equally support a transverse electromagnetic (TEM) mode, their propagation is described by a one-dimensional Helmholtz equation, with generic solution of the form [8] [9]: 


\begin{tabular}{|c|c|}
\hline \multirow{3}{*}{ Electric lines } & $V_{e}(z)=V_{e}(0) e^{-\gamma z}\left(1+\Gamma e^{2 \gamma z}\right)$ \\
\cline { 2 - 3 } & $I_{e}(z)=\frac{V_{e}(0)}{Z_{0}} e^{-\gamma z}\left(1-\Gamma e^{2 \gamma z}\right)$ \\
\hline \multirow{3}{*}{ Magnetic lines } & $I_{m}(z)=I_{m}(0) e^{-\gamma z}\left(1+\Gamma e^{2 \gamma z}\right)$ \\
\cline { 2 - 3 } & $V_{m}(z)=\frac{I_{m}(0)}{Z_{0}} e^{-\gamma z}\left(1-\Gamma e^{2 \gamma z}\right)$ \\
\hline
\end{tabular}

Table 12: General solution for the propagation equations

The load reflection coefficient is given by:

$$
\Gamma=\frac{Z_{L}-Z_{0}}{Z_{L}+Z_{0}}
$$

And the input impedance:

$$
Z_{\text {in }}=Z_{0} \frac{Z_{L}+Z_{0} \tanh (\gamma l)}{Z_{0}+Z_{L} \tanh (\gamma l)}
$$

The complex power flow per unit length of the line can be locally determined through:

\begin{tabular}{|c|c|}
\hline \multirow{3}{*}{ Electric lines } & $P_{f}^{\prime}(z)=V_{e}(z) I_{e}^{*}(z)=\frac{\left|V_{e}(z)\right|^{2}}{Z_{0}^{*}}=P_{f}^{\prime}(0) e^{-2 \alpha z}\left[\frac{V A}{m}\right]$ \\
\cline { 2 - 3 } & $P_{f}^{\prime}(0)=\frac{\left|V_{e}(0)\right|^{2}}{Z_{0}^{*}}\left[\frac{V A}{m}\right]$ \\
\hline \multirow{2}{*}{ Magnetic lines } & $P_{f}^{\prime}(z)=I_{m}(z) V_{m}{ }^{*}(z)=\frac{\left|I_{m}(z)\right|^{2}}{Z_{0}^{*}}=P_{f}^{\prime}(0) e^{-2 \alpha z}\left[\frac{V A}{m}\right]$ \\
\cline { 2 - 2 } & $P_{f}^{\prime}(0)=\frac{\left|I_{m}(0)\right|^{2}}{Z_{0}^{*}}\left[\frac{V A}{m}\right]$ \\
\hline
\end{tabular}

Table 13: Complex power flow per unit length for electric and magnetic TLs

Or, in terms of time-average active and reactive power per unit length: 


$$
\begin{gathered}
P^{\prime}(z)=\operatorname{Re}\left\{P_{f}^{\prime}(z)\right\}\left[\frac{W}{m}\right] \\
Q^{\prime}(z)=\operatorname{Im}\left\{P_{f}^{\prime}(z)\right\}\left[\frac{V A R}{m}\right]
\end{gathered}
$$

The power dissipation per unit length $P_{d}^{\prime}$ is equal to the rate of decrease in the active power per unit length $P^{\prime}$ :

$$
P_{d}^{\prime}(z)=-\frac{d P^{\prime}(z)}{d z}=2 \alpha P^{\prime}\left[\frac{W}{m}\right]
$$

Finally, the gain $G$ of the line is defined as:

$$
G=\operatorname{sqrt}\left(\frac{P^{\prime}(0)}{P_{f}^{\prime}(-l)}\right)=S_{21}
$$

\section{6.}

\section{Free-space virtual magnetic lines}

If there is no ferroelectric or ferromagnetic material channeling the flux from the source to the load, the propagation equations of the virtual lines must be adapted to compensate the flux leakage. It is intuitive that the reflection coefficient $\Gamma$ must increase inversely with the coupling between the terminals of the TL:

\begin{tabular}{|c|c|}
\hline \multirow{2}{*}{$\begin{array}{c}\text { VELTL in free } \\
\text { space }\end{array}$} & $V_{e}(z)=V_{e}(0) e^{-\gamma_{e, v} z}\left(1+\frac{\Gamma_{e, v}}{\kappa_{e}} e^{2 \gamma_{e, v} z}\right)$ \\
\cline { 2 - 2 } & $I_{e}(z)=\frac{V_{e}(0)}{Z_{m, e, 0}} e^{-\gamma_{e, v} z}\left(1-\frac{\Gamma_{e, v}}{\kappa_{e}} e^{2 \gamma_{e, v} z}\right)$ \\
\hline \multirow{2}{*}{$\begin{array}{c}\text { VMGTL in free } \\
\text { space }\end{array}$} & $I_{m}(z)=I_{m}(0) e^{-\gamma_{m, v} z}\left(1+\frac{\Gamma_{m, v}}{\kappa_{m}} e^{2 \gamma_{m, v} z}\right)$ \\
\cline { 2 - 2 } & $V_{m}(z)=\frac{I_{m}(0)}{Z_{m, v, 0}} e^{-\gamma_{m, v} z}\left(1-\frac{\Gamma_{m, v}}{\kappa_{m}} e^{2 \gamma_{m, v} z}\right)$ \\
\hline
\end{tabular}

Table 14: Adapted solutions to compensate the flux leakage 
where $\kappa_{e}$ and $\kappa_{m}$ are the electric and magnetic coupling coefficient, respectively.

The electric coupling coefficient is defined as the ratio between the mutual capacitance $C_{m}$ and the geometric mean of the self-capacitance of the terminals:

$$
\kappa_{e}=\frac{C_{m}}{\sqrt{C_{1} C_{2}}}
$$

The magnetic coupling coefficient is defined as the ratio between the mutual inductance $L_{m}$ and the geometric mean of the self-inductances of the terminals:

$$
\kappa_{m}=\frac{L_{m}}{\sqrt{L_{1} L_{2}}}
$$

The coupling coefficient is a sort of measure of how much EM modes in the near field of the terminals got coupled in relation to all generated modes.

Finally, for circuits fed by voltage sources, the terms $V_{e}(0)$ and $I_{m}(0)$ can be determined from the following equations:

$$
\begin{gathered}
V_{e, c}^{i n}=V_{\text {source }} \frac{Z_{\text {in }}}{\left(Z_{\text {in }}+Z_{\text {source }}\right)} \\
I_{e, c}^{i n}=\frac{V_{e, c}^{i n}}{Z_{\text {in }}} \\
V_{e}(0)=\frac{V_{e, c}^{i n}}{e^{\gamma_{e, v} l}\left(1+\frac{\Gamma_{e, v}}{\kappa_{e}} e^{-2 \gamma_{e, v} l}\right)} \\
I_{m}(0)=\frac{V_{e, c}^{i n}}{e^{\gamma_{m, v} l}\left(1+\frac{\Gamma_{m, v}}{\kappa_{m}} e^{-2 \gamma_{m, v} l}\right)}
\end{gathered}
$$




\section{4}

\section{Implementation of virtual impedances}

In this chapter, it is demonstrated that MTMs can be seen as virtual impedances when coupled to virtual lines and can be used to improve the impedance matching between a virtual line and its terminals.

Considering that only the implementation of the magnetic model is of interest for the realization of this work, its electrical counterpart will not be further addressed. However, the same reasoning and principles applied to VMGTLs is valid and could be adapted to VELTLs.

\section{1.}

\section{Introduction}

In 1968, Veselago presented a theoretical study on EM wave propagation in media with negative properties [24]. He demonstrated that materials with just one of its EM properties effectively negative, such as the plasmas $-\varepsilon$-negative (ENG) and $\mu$-negative (MNG) materials -, supported no propagating mode but that hypothetical materials whose EM properties were double negative (DNG) could support wave propagation just as double positive (DPS) ones, such as the dielectrics (see Figure 4.1). 


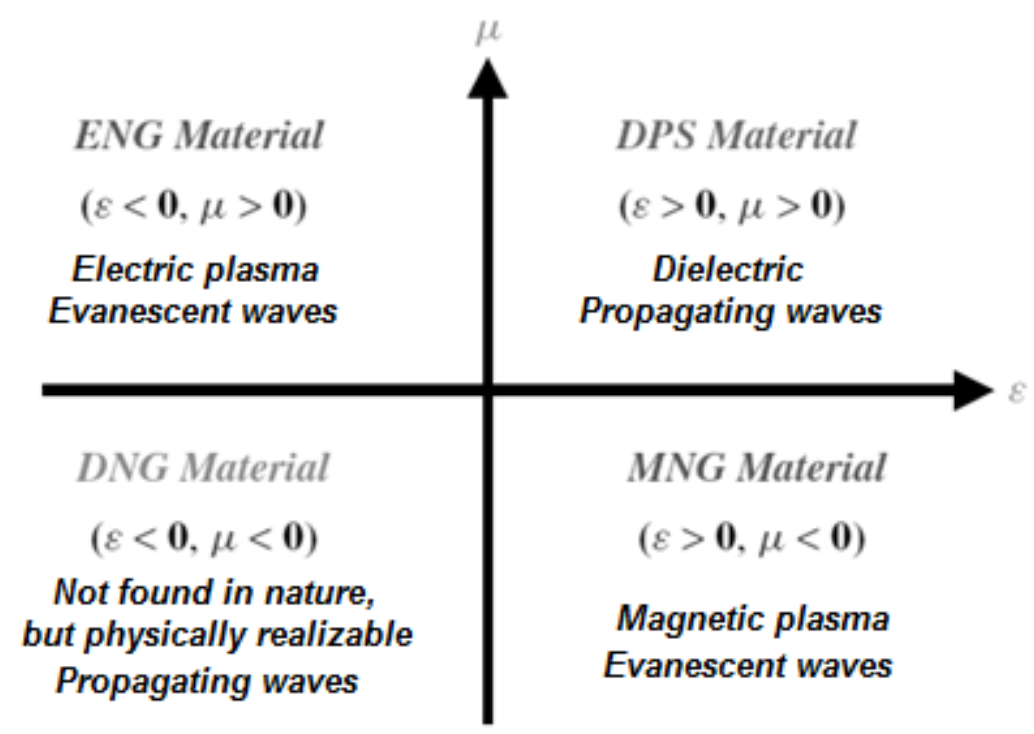

Figure 4.1: Classification of materials according to the sign of its properties

According to Veselago's predictions, NIMs would present the exotic property of negative refraction:

$$
\begin{gathered}
n=n^{\prime}-j n^{\prime \prime} \\
n=\sqrt{|\varepsilon|} \sqrt{-|\mu|}=(\sqrt{|\varepsilon|})(j \sqrt{|\mu|})=j \sqrt{|\varepsilon \mu|} \\
n=\sqrt{-|\varepsilon|} \sqrt{|\mu|}=(j \sqrt{|\varepsilon|})(\sqrt{|\mu|})=j \sqrt{|\varepsilon \mu|} \\
n=\sqrt{-|\varepsilon|} \sqrt{-|\mu|}=(j \sqrt{|\varepsilon|})(j \sqrt{|\mu|})=-\sqrt{|\varepsilon \mu|}
\end{gathered}
$$

where $n$ is the complex refractive index, $n^{\prime}$ is the refractive index and $n^{\prime \prime}$ is the extinction coefficient.

And also the phenomenon of reverse propagation:

$$
\begin{gathered}
-\nabla \times \boldsymbol{E}=j \omega(-|\boldsymbol{\mu}|) \boldsymbol{H} \rightarrow-\nabla \times \boldsymbol{E}=j \omega|\boldsymbol{\mu}|(-\boldsymbol{H}) \\
\nabla \times \boldsymbol{H}=j \omega(-|\boldsymbol{\varepsilon}|) \boldsymbol{E} \rightarrow \nabla \times(-\boldsymbol{H})=j \omega|\boldsymbol{\varepsilon}| \boldsymbol{E} \\
\therefore \boldsymbol{k} \perp \boldsymbol{E} \perp(-\boldsymbol{H}) \rightarrow-\boldsymbol{k} \perp \boldsymbol{E} \perp \boldsymbol{H}
\end{gathered}
$$




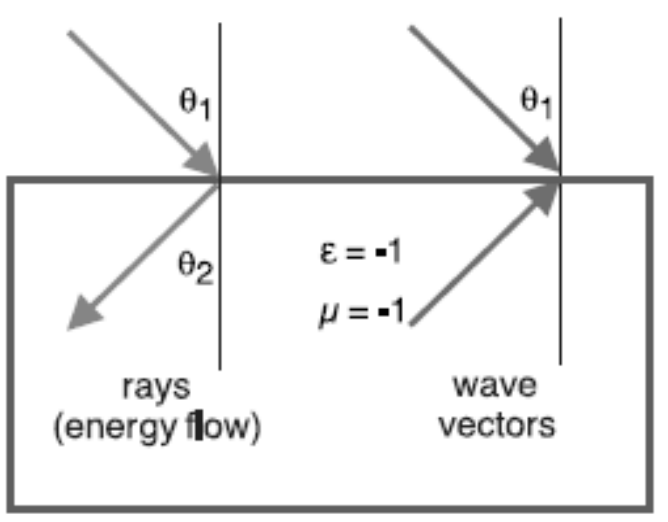

Figure 4.2: In negative-index media the phase velocity is reversed in relation to the direction of the power flow

Since the relationship between the fields and wave vector is given by a left-hand rule, NIMs are said to be left-handed materials (LHM). Or backward-wave materials (BWM), due to the fact that phase velocity becomes antiparallel to the direction of the power flow if $n<0$ (see Figure 4.2):

$$
\begin{gathered}
\operatorname{dir}\left(v_{p}\right)=\operatorname{dir}\left(\frac{\omega}{\boldsymbol{k}}\right)=\operatorname{dir}(\boldsymbol{k}) \\
\operatorname{dir}\left(v_{s}\right)=\operatorname{dir}(\overline{\boldsymbol{S}})=\operatorname{dir}\left(\boldsymbol{E} \times \boldsymbol{H}^{*}\right)=\operatorname{dir}(-\boldsymbol{k})
\end{gathered}
$$

Another prediction of Veselago's study was that NIMs would present perfect lensing capabilities, this is to say, lenses made of NIMs would be able to focus not only the propagating modes (far field) but also the evanescent ones (near field). Differently of traditional lenses in which all the information conveyed by the evanescent modes is lost (originating the so-called diffraction limit), in a flat lens made of NIM, all the information carried by all modes would be recovered [25]. 

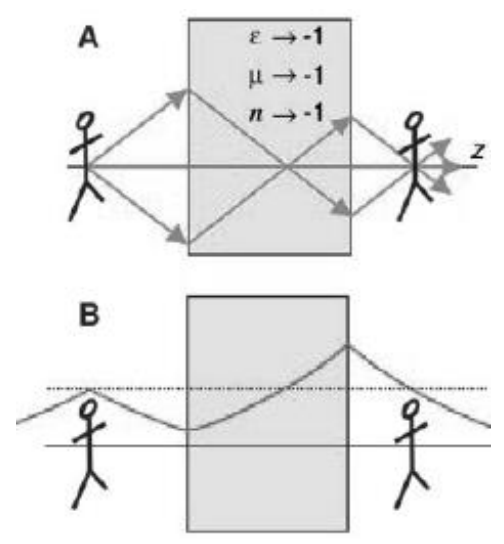

Figure 4.3: Perfect lensing implies the focalization of the far field (A) and the near field (B)

As it can be seen in Figure 4.3, the evanescent mode becomes "growing exponentials" inside a NIM, implying that evanescent modes are focusing by means of local amplification of the near field.

As no material with negative response was found by the time of Veselago's publication, his ideas remained only as a theoretical curiosity until 2000, when it was demonstrated by John Pendry and David Smith that an artificial complex media based on periodic structures, later known as metamaterials, could present a frequency band over which $\varepsilon$ and $\mu$ are both negative [26] [27] [28].

The promise of physically realizable artificial materials capable of synthesizing new response functions that do not occur in nature rapidly evolved to the idea of artificial structures that could mimicking any response functions (including those of known materials that may not be easily available). That concept opened a whole new frontier, not only for electrical engineers, but also for mechanical and materials ones.

\section{2.}

\section{Metamaterials}

In electromagnetics, a metamaterial (MTM) is an artificial material whose effective EM properties can be controlled by designing circuits that serve as "artificial atoms". Usually, they are periodic structures in which the response function to an incident EM depends on both: 1) the EM behavior of the unit-cell ("the atoms") and their geometric arrangement in 
space ("the crystalline lattice") [14] [15]. A MTM could then be though as an "artificial crystal" made of engineered atoms and lattices (see Figure 4.4).

Based on the effective medium theory, those structures can be mathematically described by equivalent homogeneous media with effective EM properties if the inter-inclusion distances of their lattice and their physical dimensions are much smaller than the wavelength $\lambda$ of the incident EM fields [29] [30].

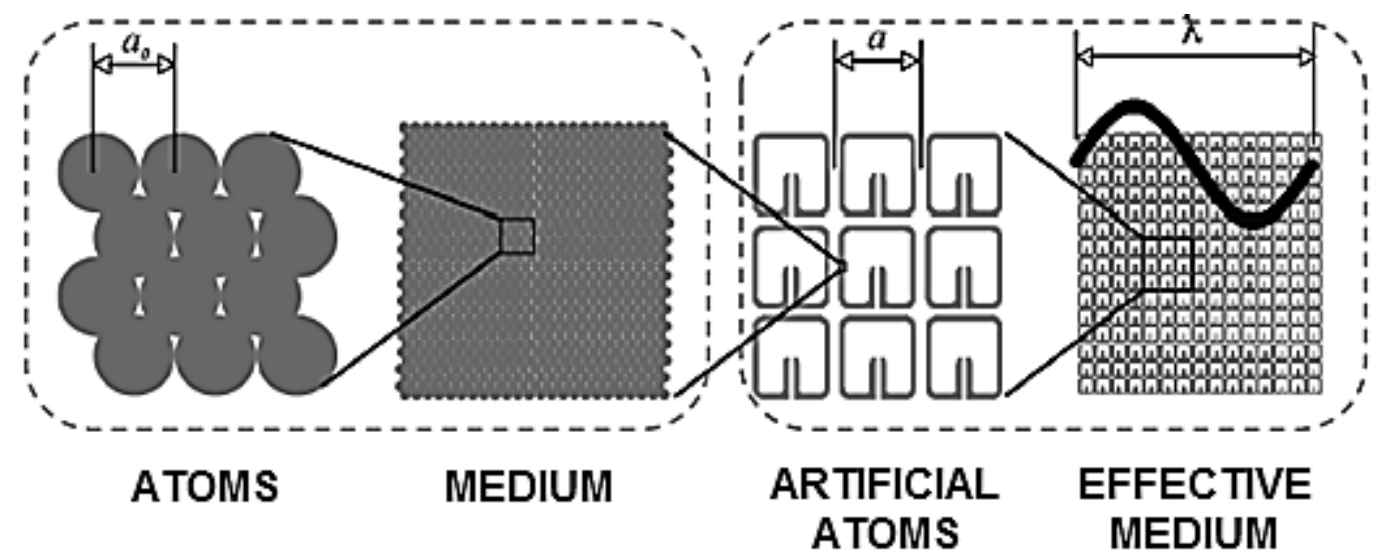

Figure 4.4: MTMs are artificial crystals made of engineered atoms and lattice

A huge variety of fascinating devices and applications relying in the MTM concept have been proposed since the publication of pioneering work of Pendry and Smith [31]. Here in this work, however, the only MTM of interest are those synthesizing structures capable of almost perfect lensing (artificial NIM).

\section{3.}

\section{Realization of negative-index materials}

Passive NIMs are realizable only over a limited bandwidth (see Section 2.8). As shown in [32], a split-ring resonator (SRR) or spiral resonator (SR) based cell has a response function for which $\mu<0$ around the resonance frequency of the unit-cell while a TL based cell has a response for which $\varepsilon<0$ below its cutoff frequency. 
Hence, by well choosing the parameters of the lattice of TL unit-cells in a way that its cutoff frequency is significantly above the resonance frequency of the lattice of SRR or SR unit-cells, a DNG MTM can be synthesized from a composite of two SNG MTMs in different planes: the $\varepsilon$ negative MTM in the E-plane and the $\mu$-negative one in the $\mathrm{H}$-plane (see Figure 4.5).

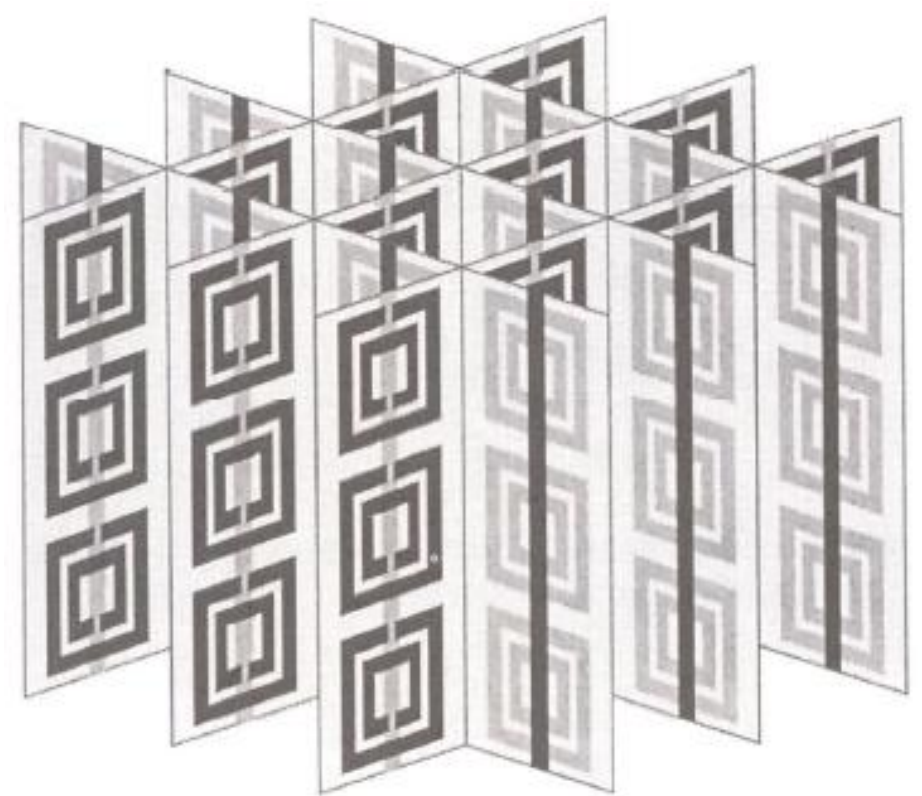

Figure 4.5: DNG structure made out of SNG MTMs in different planes

\subsection{1.}

$\varepsilon$-negative metamaterial

Let's consider the $\varepsilon$-negative MTM first. As stated in Section 2.3.1, materials presenting $\varepsilon<0$ are very negatively polarized, which is the case of any metal, since it is always polarized in opposition to the incoming electric field (producing an effective zero electric field at the metal surface). So, concerning the $\varepsilon$-negative MTM, the restriction is to reduce the metallic surface to a minimum without losing the negative response at the frequency of interest. 


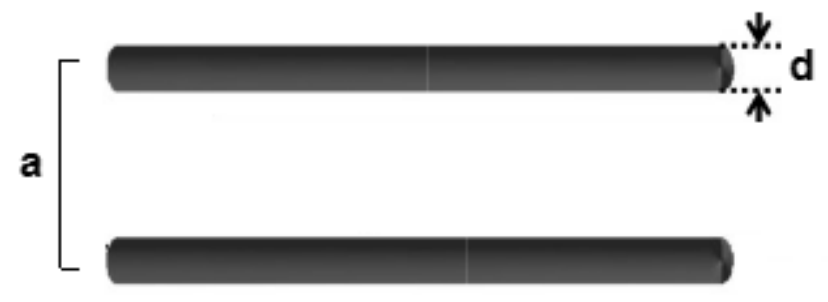

Figure 4.6: TL-based cell

The plasma frequency of the $\varepsilon$-negative MTM will diminish with the width of the metal sheet, as well as the intensity of its negative response in the entire frequency spectrum. The two major parameters of design control are the diameter $d$ of the unit-cells and their periodicity a (see Figure 4.6).

\subsection{2.}

\section{$\mu$-negative metamaterial}

Now concerning the design of the $\mu$-negative MTM. As discussed in Section 2.3.2, for magnetic materials a negative response implies a material with highly diamagnetic behavior, which is basically occurs when its atoms are magnetized by EM induction (the magnetic moment of the atoms oppose the incoming magnetic moment). So, the intensity of negative response of the $\mu$-negative MTM depends on "how resilient" its meta-atoms are to EM induction, which implies they must conserve their stored energy as long as possible.

Then, in order to delay the changing in their magnetization state, the circuitry of the MTM unit cells must present a high quality factor ( $Q$-factor).

The Q-factor is a dimensionless parameter that measures how under-damped is a resonator [9]. It is defined as the ratio between the time-average stored energy and the power dissipated per cycle:

$$
Q \triangleq \omega \cdot \frac{\bar{U}}{P_{d}}
$$


As demonstrated in [33], for equivalent SRR and SR based cells, with the same value of turns, the Q-factor of the SR model is 2 or 3 times greater than the Q-factor of SRR one (see Figure 4.7). Since the $\mu$ negative response of the MTM depends basically on Q-factor of its cells, the SR resonator is more efficient than an equivalent SRR. Besides that, the linear dimension of the outer length of a SR can be even 5 times shorter than the one of a SRR, which makes the SR cells also more suitable for miniaturization.

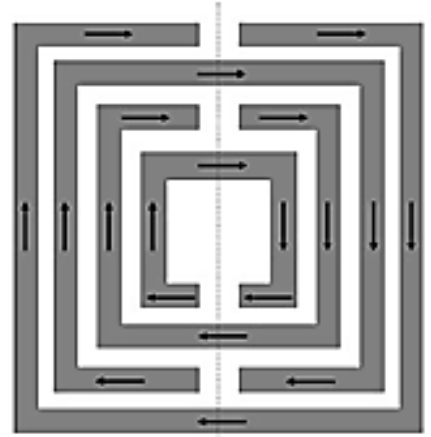

(a)

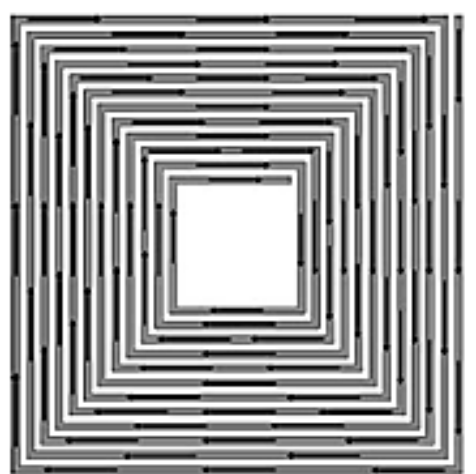

(b)

Figure 4.7: (a) Split-ring and (b) spiral resonator based cells

Considering the particular case of VMGTLs, the operating distance $D$ is expected to be large (in the order of meters, at least). So, the working frequency $f_{0}$ should be in the order of $\mathrm{kHz}$ or $\mathrm{MHz}$ (due to the theoretical limit of the near-field zone). However, considering that the MTM lenses are supposed to work inside electronic devices as small as a smartphone, the achieved resonance frequency will be in the order of $\mathrm{GHz}$ or greater for any practical unit-cell size.

Thus, the control of the resonance frequency must be made by adding more capacitance or inductance to the unit-cell coil. For example, by adding interdigital capacitors or lumped elements to it (see Figure 4.8). 


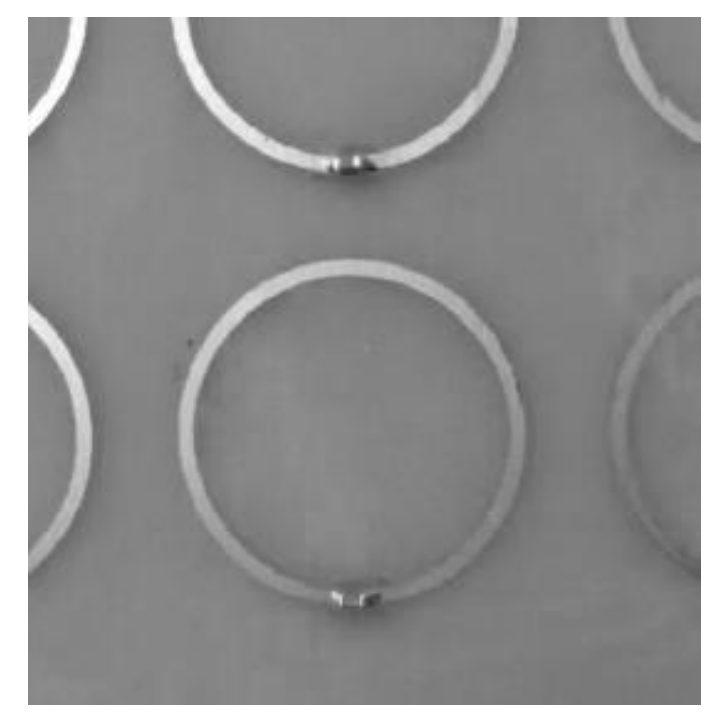

Figure 4.8: Resonance control with lumped capacitors

SR inclusions behave like a parallel LC circuit if only stray capacitance is considered. On the other hand, they behave as a series LC circuit when a lumped capacitor is embedded to their terminals.

This fact has an important consequence concerning Q-factor:

$$
\begin{gathered}
Q_{\text {series }}=\frac{\omega_{0} L}{R}=\frac{1}{R} \sqrt{\frac{L}{C}} \\
Q_{\text {parallel }}=\frac{R}{\omega_{0} C}=R \sqrt{\frac{C}{L}}
\end{gathered}
$$

So, if the inclusions form a parallel RLC circuit, $Q$-factor increases with the square root of the capacitance and decreases with the square root of the inductance, and the opposite happens if they behave as a series RLC circuit. In the parallel case, the operating frequency of the circuit must be diminished by augmenting its capacitance, while in the series one, it is the circuit's inductance that must be enhanced, in order to avoid that the frequency adjustment degrades the $\mathrm{Q}$-factor.

NIMs are useful concerning the main topic of this dissertation because the magnetic circuit or VMGTL formed between the coils are due to the coupling of the evanescent EM modes presenting in their near fields 
(see Section 2.10). By focusing the EM evanescent modes with a NIM-like slab, the overall efficiency of a VMGTL can be enhanced (see Figure 4.9).

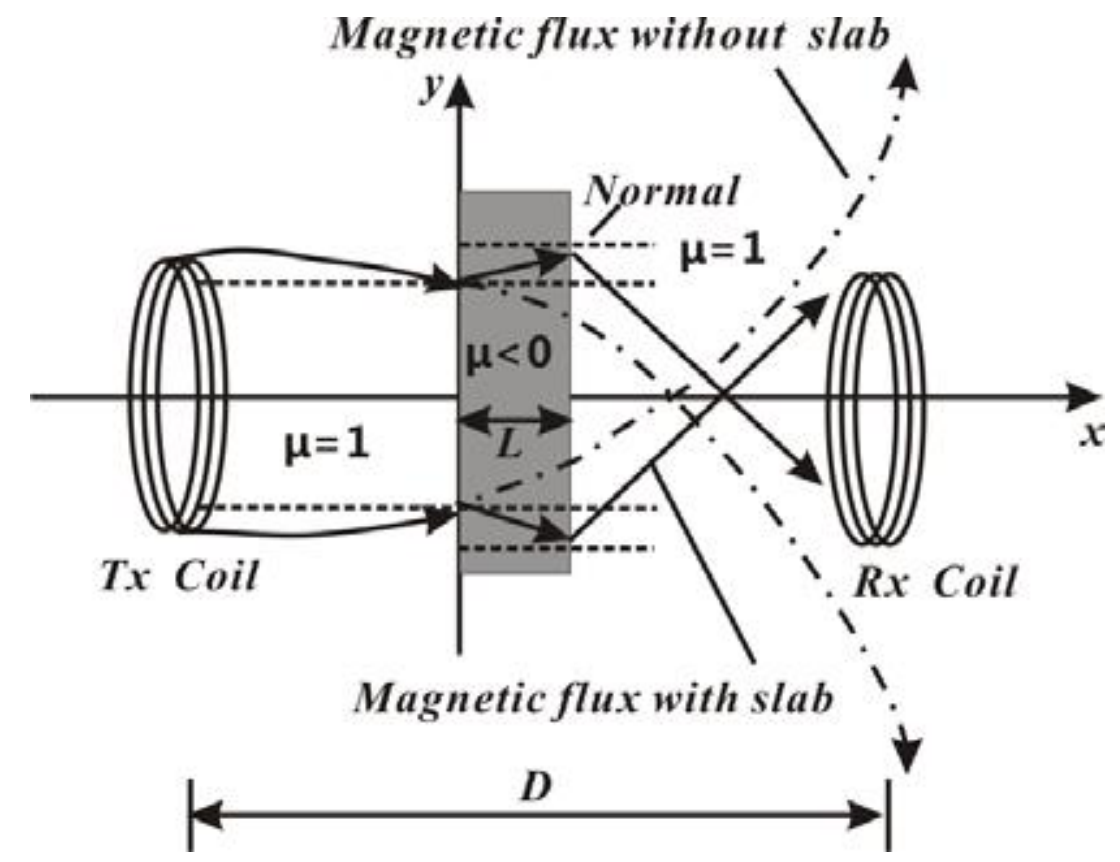

Figure 4.9: The focalization of the evanescent modes enhances the magnetic coupling

This phenomenon, known as "MTM-enhanced magnetic coupling", is exhaustively addressed in the literature [7] [5] [34] [35]. Nonetheless, the "enhanced coupling" interpretation is a kind of obscure concept, since it does not reveal in details the underlying mechanism governing the power transfer gain introduced by the MTM.

As it is the intention of this dissertation to demonstrate, by applying the generalized theory of TLs to such systems, the "evanescent-mode amplification" and the subsequent enhancement of the power transfer through the magnetic link can be explained as an improvement in the impedance matching of the VMGTL with its terminals (which are ELTLs).

In a VMGTL, seen that the evanescent EM modes are mostly magnetic ones, only the magnetic permeability $\mu$ needs to be effectively negative to produce the amplification effect. Nonetheless, despite the fact that single-negative (SNG) lenses are easier to implement, they impose more severe losses to the system once SNG structures do not support propagating modes. 
As it was discussed in Chapter 3, the real power transmitted from one physical circuit to the other by means of a VMGTL is transported by guided modes confined between the virtual currents (time-varying fluxes), not by the fluxes themselves, analogously to the ELTL problem, where the conduction currents of the line transport no power, only dissipate it as heat. The fluxes are reactive in nature and carry no real power, only store energy in the intervening space.

So, DNG lenses will present a better performance than the SNG ones, even though the amplification of magnetic evanescent modes depends only on how negative is $\mu$, because they will not interfere destructively with the guided modes of the VMGTL. As it is discussed in [15] [14], at least in theory, DNG structures can be lossless, while SNG ones are always lossy. In practice, it means that losses are much smaller when the MTM is DNG.

\section{4 .}

\section{Proposed prototype for a $\mu$-negative metamaterial}

For the sake of simplicity, the proposed prototype is a SNG structure synthesizing a $\mu$-negative MTM. Nonetheless, all the results concerning the VMGTL problem can be generalized for DNG structures as well.

\subsection{1.}

\section{Parameters}

The proposed prototype consists in artificial crystalline lattice made of square SR resonators built over a double-faced metallized printed circuit board (PCB) made of FR4 and cooper. The frequency regulation is made by mean of CMS capacitors $\left(C_{C M S}\right)$ on the backside of the slab connected in series with the resonators in the front side via two metallic holes (see Figure 4.10). 


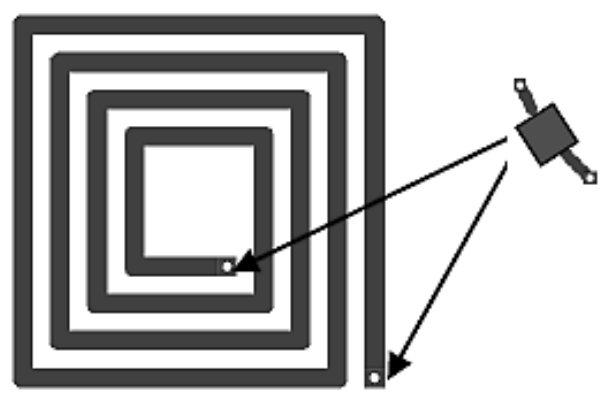

Figure 4.10: Proposed unit cell

Prototype characteristics:

- $d_{\text {int }}=1 \mathrm{~cm}$

- $d_{\text {ext }}=2 \mathrm{~cm}$

- $s=1 \mathrm{~mm}$

- $\quad w=1 \mathrm{~mm}$

- $C_{C M S}=100 p F$

- $a=2.3 \mathrm{~cm}$

- $\quad N=4$

where $w$ is the strip width, $s$ is the separation between two adjacent rings, the $l$ is the side length of the outer ring, a is the period of the lattice and $N$ is the number of turns of the coil.

Electric characteristics of the PCB used in the project:

- $\rho=10 \mu \Omega . m m$

- $\varepsilon_{r}=5.4$

- $\delta_{e}=0.01$

- $e_{d}=1.0 \mathrm{~mm}$

- $e_{m}=35 \mu m$

where $e_{d}$ and $e_{m}$ are the thickness of the dielectric and the metallic surfaces of the slab, respectively.

Knowing that the total length $l$ of the conductor of the unit cell and its section $A$ are: 
- $\quad l=179 \mathrm{~mm}$

- $A=0,017 \mathrm{~mm}^{2}$

Its electrical resistance can be estimated as:

- $R=0.2 \Omega$

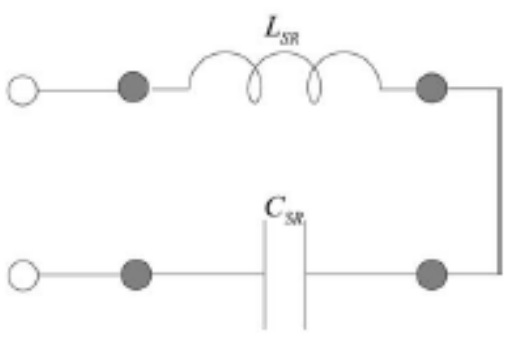

Figure 4.11: Equivalent circuit of a SR-based cell

Based on the equivalent model of the square SR-based cell in the quasistatic regime, its total inductance can be estimated by the following equations [33] (see Figure 4.11):

$$
\begin{gathered}
L_{S R}=\frac{\mu_{0}}{2 \pi} l_{\text {avg }}^{S R}\left[\frac{1}{2}+\ln \left(\frac{l_{\text {avg }}^{S R}}{2 w}\right)\right] \\
l_{\text {avg }}^{S R}=\frac{4 l N-[2 N(1+N)-3](w+s)}{N}
\end{gathered}
$$

where $l_{a v g}^{S R}$ is the single turn average length.

- $L_{S R}=240 \mathrm{nH}$

Ignoring the stray capacitance of the unit cell, its resonance frequency and the $\mathrm{Q}$-factor are estimated by:

- $f_{0}=\frac{1}{2 \pi \sqrt{L_{S R}\left(C_{C M S}+C_{S R}\right)}}=32.4 \mathrm{MHz}$ 
- $Q=\frac{1}{R} \sqrt{\frac{L_{S R}}{C_{C M S}}}=245$

The total external dimension of the MTM slab is $20 \mathrm{~cm} \times 20 \mathrm{~cm}$ (see Figure 4.12 and Figure 4.13).

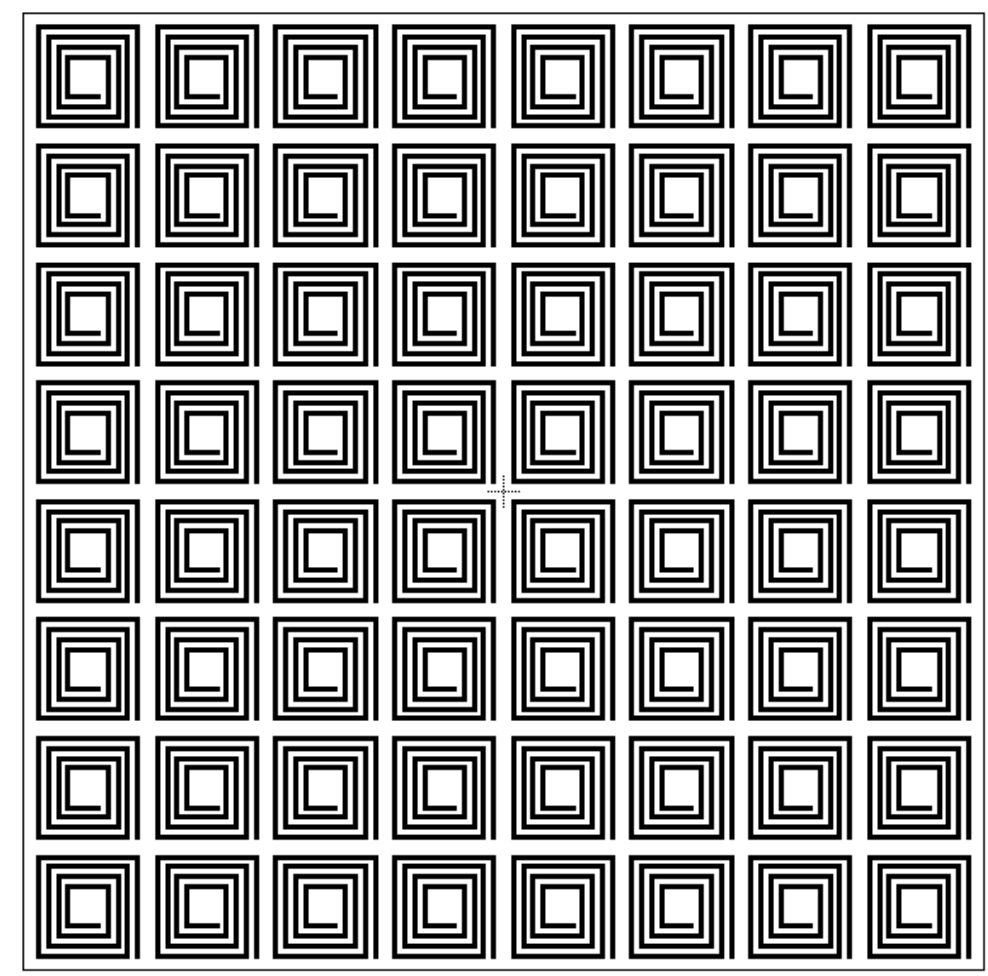

Figure 4.12: Representation of the front side of MTM prototype 


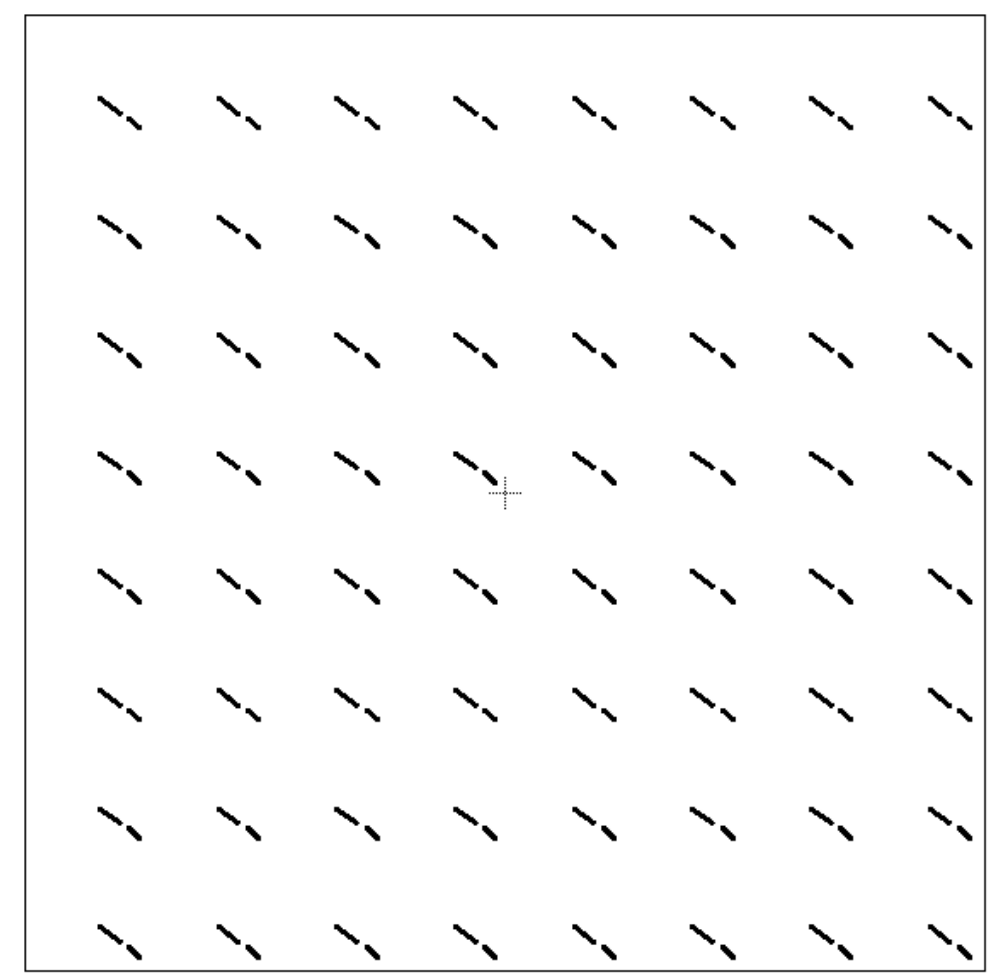

Figure 4.13: Representation of the back side of MTM prototype

\subsection{2.}

\section{Retrieval of the effective permeability}

Based on the analytic description of the EM properties presented in Section 2.3, the Lorentz model can be used to estimate the relative permeability $\mu_{r}$ of the MTM [36]:

$$
\begin{gathered}
\mu_{r, M T M}=1+\frac{F \omega^{2}}{\omega^{2}-j \omega \xi-\omega_{0}^{2}} \\
\xi=\frac{\omega_{0}}{Q}
\end{gathered}
$$

where $F$ is the coupling coefficient between adjacent cells of the lattice and $\xi$ is the damping ratio of the system. Using the mutual inductance estimation giving by [37], the coupling coefficient $F$ can be determined by:

$$
F=\frac{N \mu_{0} \pi d_{e x t}^{4}}{4 L_{0} a^{2} \sqrt{4 d_{e x t}^{2}+a^{2}}}=0.43
$$




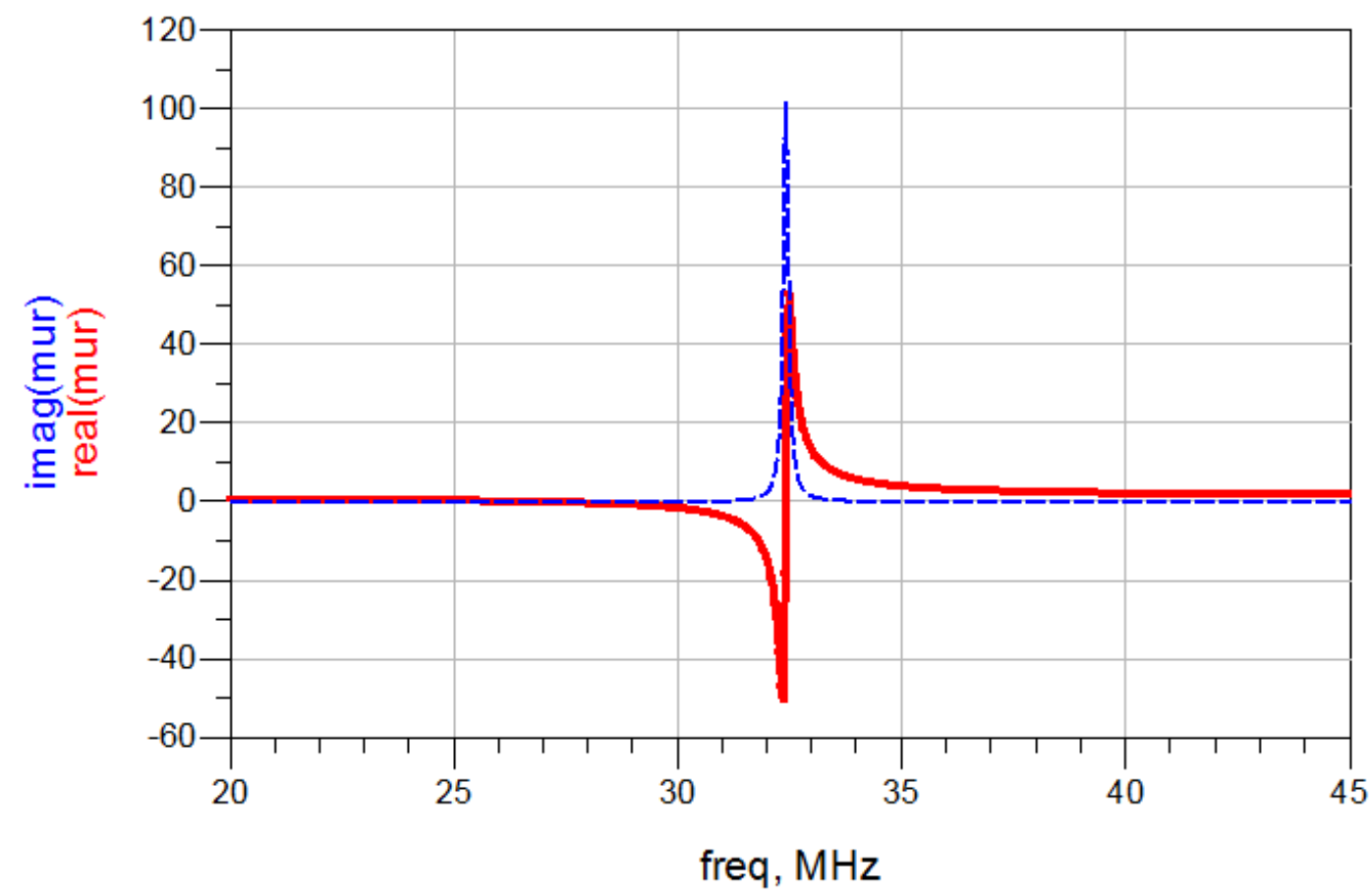

Figure 4.14: Estimation of the real (red solid) and imaginary part (blue dash) of $\mu_{r}$

As it can be seen in Figure 4.14, $\mu_{r}$ becomes negative around the resonance frequency $\left(f_{0}=32.4 \mathrm{MHz}\right)$ :

$$
\mu_{r, M T M_{\text {analytical }}}=-51-j 101.6
$$

\subsection{3.}

\section{Equivalent virtual impedance}

The equivalent inductance of the MTM can be determined from its effective parameters:

$$
L_{M T M}=\mu_{r, M T M} \mu_{0} a=\left(1+\frac{F \omega^{2}}{\omega^{2}-j \omega \xi-\omega_{0}^{2}}\right) \mu_{0} a
$$

Thus, MTM equivalent reactance is given by (see Figure 4.15): 


$$
X_{M T M}(\omega)=\omega L_{M T M}
$$

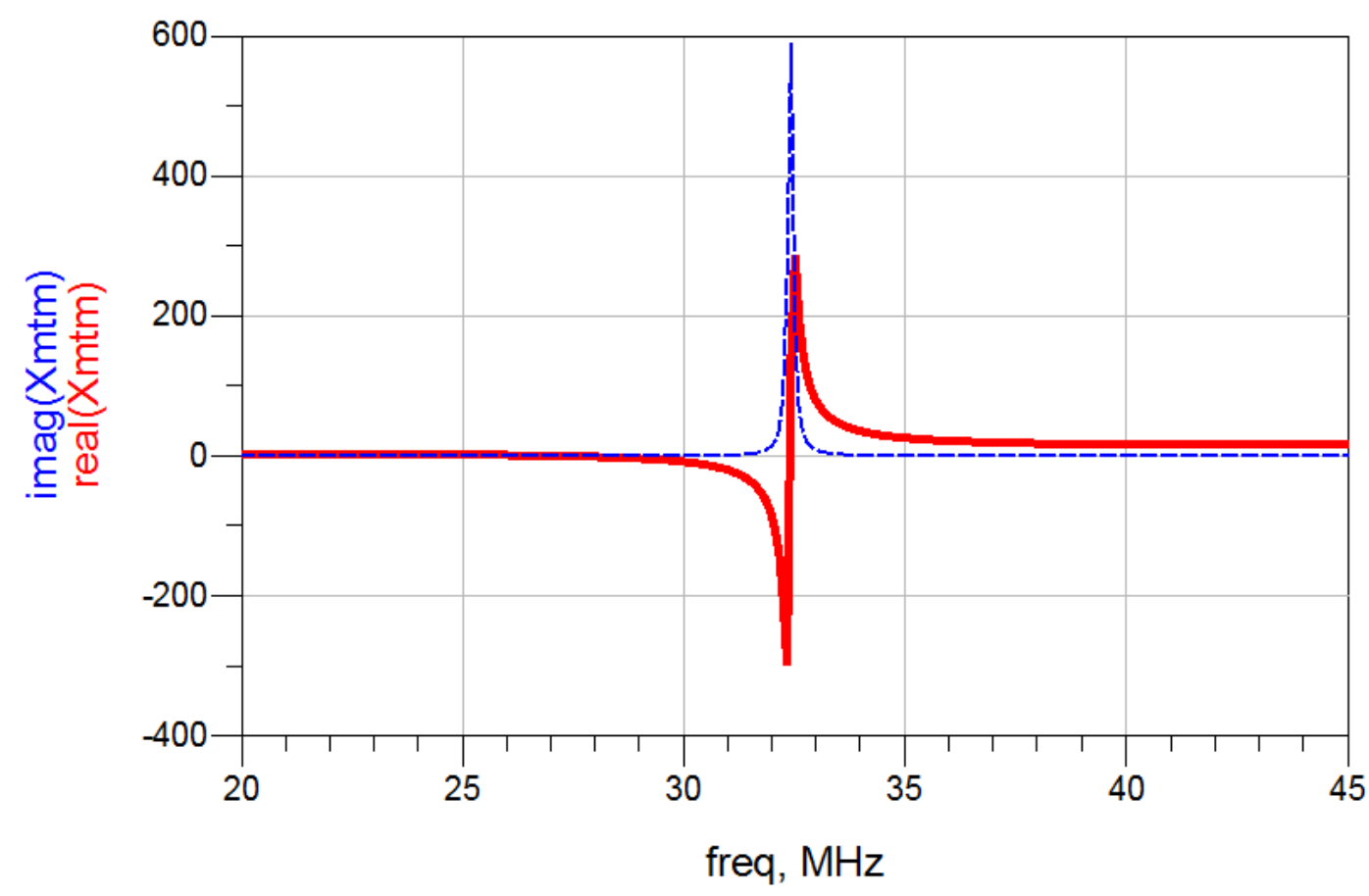

Figure 4.15: Reactance $X_{M T M}$ as a function of frequency

Accordingly to Foster's reactance theorem, the reactance of a passive lossless two-terminal networks increases monotonically with frequency, which means that its first derivative in relation to frequency is always positive [38]:

$$
\begin{gathered}
X_{L}=\omega L \rightarrow \frac{d Z_{L}}{d f}=2 \pi L>0, \quad \text { if } L>0 \\
X_{C}=-\frac{1}{\omega C} \rightarrow \frac{d Z_{C}}{d f}=\frac{1}{2 \pi f^{2} C}>0, \quad \text { if } C>0
\end{gathered}
$$




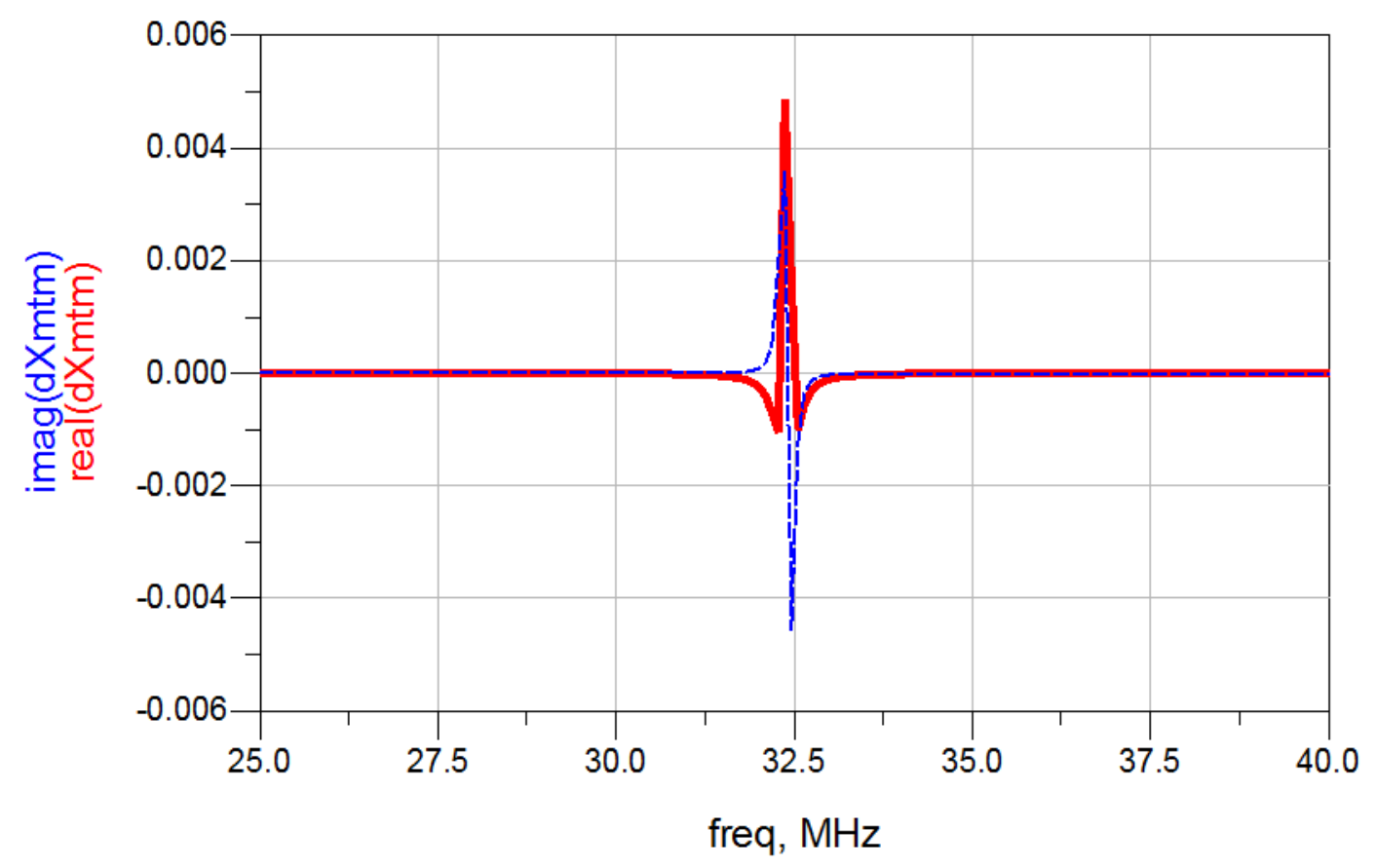

Figure 4.16: First frequency derivative of $X_{M T M}$

A positive derivative for the impedance is a stability condition of passive circuits. An impedance that has a negative derivative is named non-Foster and describes a gain element. For a passive element like the MTM to present a non-Foster behavior, it must be frequency-limited. As it can be seen in Figure 4.16, the MTM equivalent reactance presents a negative derivative over a small bandwidth around $f_{0}$. This bandwidth limitation of the MTM as a gain medium is a direct consequence of the causality condition discussed for NIMs in Section 2.8.

The MTM equivalent virtual impedance is given by:

$$
Z_{m, v}(\omega)=j X_{M T M}(\omega)=j \omega L_{M T M}(\omega)
$$




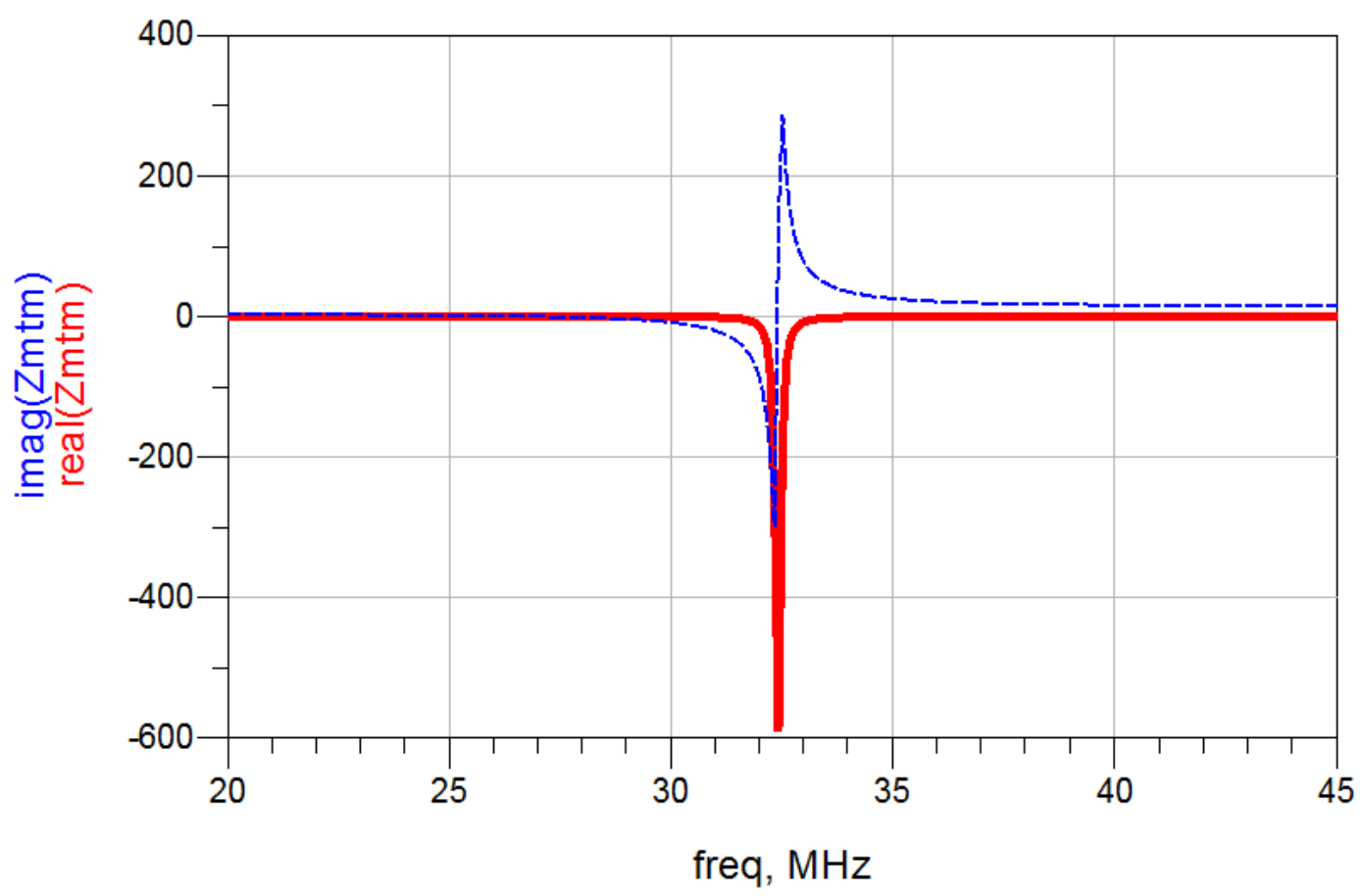

Figure 4.17: MTM equivalent impedance $Z_{m, v}$ as a function of frequency

As it is shown in Figure 4.17, the MTM is perceived as negative resistance around the resonance $\left(z_{m, v} \in \mathbb{R}^{-}\right)$. At the range of sub-resonance frequencies, it is completely invisible to the virtual line $\left(z_{m, v}=0\right)$. And at the over-resonance one, it is seen as a positive reactance $\left(z_{m, v} \in \mathrm{Im}^{+}\right)$.

As a negative resistance it acts in the sense of enhancing the magnetic current (and consequently the magnetic flux) of the virtual line:

$$
\frac{d}{d z} I_{m, d}(z)=-\left|\operatorname{Re}\left\{Z_{m, v}\left(\omega_{0}\right)\right\}\right| V_{m, v}(z)
$$

As a positive reactance, the MTM becomes equivalent to an inductor and acts as storage element. The energy stored by the MTM from higher frequency modes is the energy it uses to amplify the resonance one. 


\section{5}

\section{Magnetic transmission line assisted by metamaterial}

In this chapter, it is demonstrated by means of analytic calculations and numerical simulations that a magnetic link can be satisfactorily described as a VMGTL. In the same manner, it is demonstrated that the gain associated with the interaction of MTM with the magnetic link is due to improvements in the impedance matching of the virtual line. The results are supported by experimental evidence. The simulation of the EM-field distribution of the system is also presented in order to clarify certain aspects of its behavior.

\section{1 .}

\section{Proposed system}

The proposed TL is a free-space VMGTL made of two magnetically coupled loop antennas of radius $r=5 \mathrm{~cm}$ made of copper wire with diameter $p=1 \mathrm{~mm}$. The loops operate far from self-resonance. It is assumed that the electrical resistance of wires, the capacitance $C$ and the radiation resistance of the antennas as well as the internal resistance of the source are all negligible. The secondary driver is connected to a load $R_{L}=50 \mathrm{ohms}$. The drivers are separated by a distance $D=15 \mathrm{~cm}$. The amplitude of the tension source is $V_{e, c}^{i n}=1 \mathrm{~V}$. The source loop is assumed to have a small internal resistance $R_{\text {source }}=0.5 \mathrm{Ohms}$.

The self-inductance and the self-capacitance of the loops are estimated by [39] [40]:

$$
L_{0}=2 \mu_{0} r\left(\ln \left(\frac{16 r}{p}\right)-1.75\right)=620 n H
$$




$$
\begin{gathered}
L_{1}=L_{2}=L_{0} \\
C_{0}=0.116 * p+0.16 r+0.19 \sqrt{\frac{r}{p}}=0.28 p F \\
C_{1}=C_{2}=C_{0}
\end{gathered}
$$

Considering that the intervening space between the loops is the air:

$$
\begin{gathered}
\mu=\mu_{0} \rightarrow G_{m}^{\prime}=0 \\
\varepsilon=\varepsilon_{0} \rightarrow G_{e}^{\prime}=0
\end{gathered}
$$

Since there is no material loss and the total reluctance of the VMGTL is approximately equal to:

$$
\begin{gathered}
\mathcal{R}_{m}=\operatorname{Re}\left\{\mathcal{R}_{m}\right\}=2 \frac{\left(\bar{U}_{m}-\bar{U}_{e}\right)}{\psi_{m}^{2}}=\frac{2}{L_{0}}-2 \omega^{2} C_{0} \\
\mu^{\prime \prime}=\varepsilon^{\prime \prime}=0 \rightarrow \operatorname{Im}\left\{\mathcal{R}_{m}\right\}=0
\end{gathered}
$$

where $a$ is the diameter of the antenna and $p$ is the diameter of the wire.

The coupling coefficient between the antennas can be estimated by [37]:

$$
\kappa=\frac{\mu_{0} \pi r^{4}}{L_{0} \sqrt{4 r^{2}+D^{2}} D^{2}}=0.01
$$

Considering that the source loop possesses an internal resistance, the propagation equations for the VMGTL must be rewritten as:

$$
\frac{d}{d z} V_{m, v}(z)=\left(\frac{\mathcal{R}_{m}^{\prime}}{j \omega}+j \omega C^{\prime}\right) I_{m, d}(z)
$$




$$
\frac{d}{d z} I_{m, d}(z)=\left(R_{\text {source }}+j \omega L^{\prime}\right) V_{m, v}(z)
$$

It is important to notice that in the present case, since the frequency range is too low (of the order of tens of $\mathrm{MHz}$ only), the skin effect of the wires were neglected. For circuits working in higher frequencies, however, it should be considered.

\section{2.}

\section{Analytical and numerical results for the proposed system}

The 2-port simulations of the system were made using Keysight's Advanced Design System (ADS). The simulations of the EM-field distribution of the system were made using Keysight's Electromagnetic Professional (EMPro) and Dassault Systèmes' Computer Simulation Tecnology (CST). The simulation on ADS is shown in Figure 5.1.

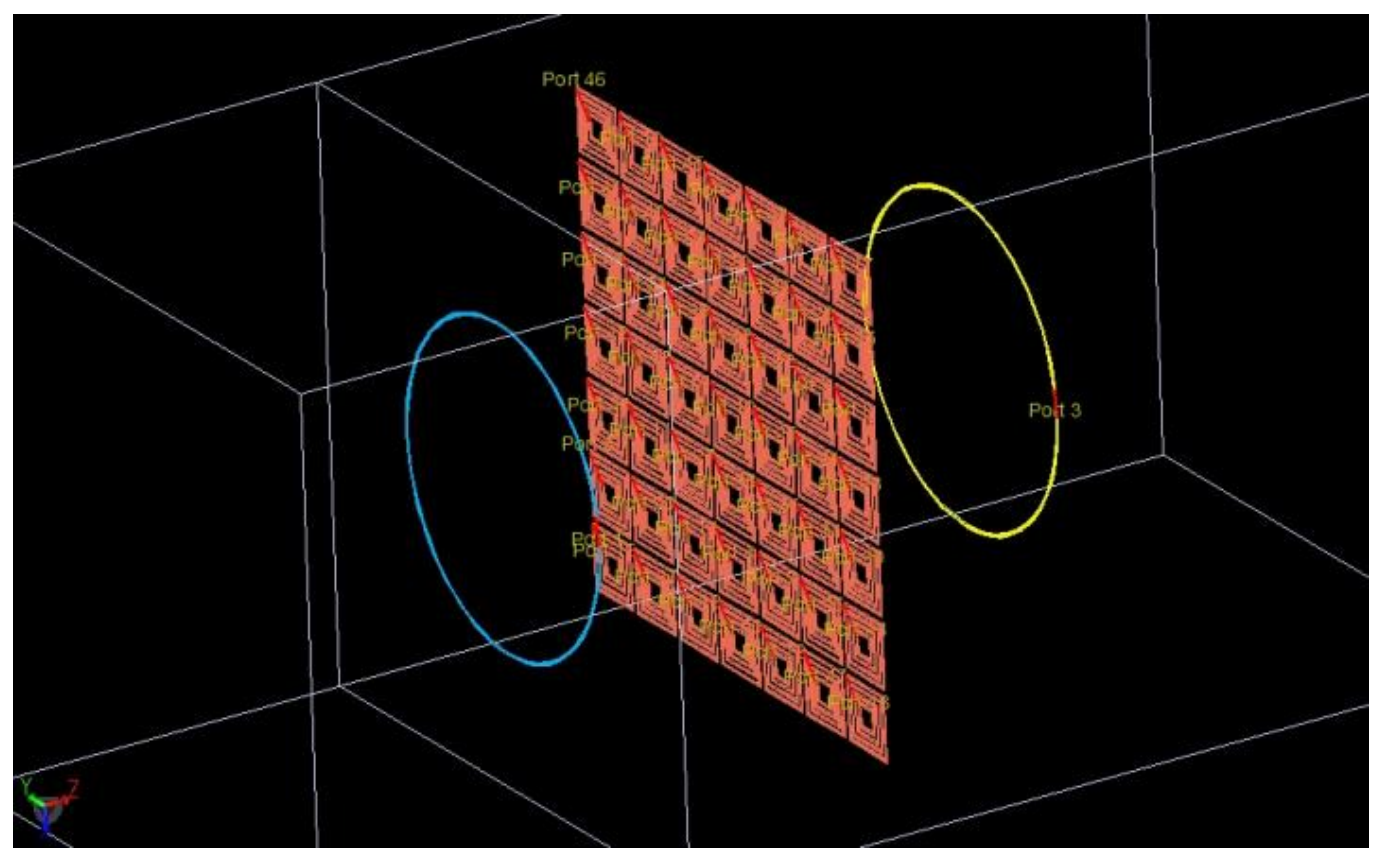

Figure 5.1: Simulation of the proposed system on ADS 


\subsection{1.}

\section{Simulated effective permeability and virtual impedance}

The equivalent $\mu_{r}$ for the MTM can be estimated from the scattering parameters of the system (S-parameters) [41]:

$$
\begin{gathered}
K=\frac{S_{11}^{2}-S_{21}^{2}+1}{2 S_{11}} \\
\Gamma=K \pm \sqrt{K^{2}-1} \\
T=\frac{S_{11}+S_{21}-\Gamma}{1-\left(S_{11}+S_{21}\right) \Gamma}
\end{gathered}
$$

There is an ambiguity of the sign of $\Gamma$. Accordingly to [41], it must be chosen in order to make $|\Gamma|<1$.

$$
\begin{gathered}
\gamma=\frac{\ln \left(\frac{1}{T}\right)}{D} \\
\mu_{r}=\frac{\gamma}{\gamma_{0}}\left(\frac{1+\Gamma}{1-\Gamma}\right) \\
\gamma_{0}=j \frac{2 \pi}{\lambda_{0}}
\end{gathered}
$$

where $D$ is the distance between the coils.

In order to compensate other effects and to extract the $\mu_{r}$ corresponding to the MTM only, one must initially estimate the $\mu_{r}$ of the system without the MTM and then rewrite the above solution for $\mu_{r}$ with the appropriated calibration:

$$
\mu_{r, M T M}=\frac{\gamma}{\gamma_{0}}\left(\frac{1+\Gamma}{1-\Gamma}\right)-\mu_{r, 0}
$$

where $\mu_{r, 0}$ represents the $\mu_{r}$ estimated for the system without the MTM. 


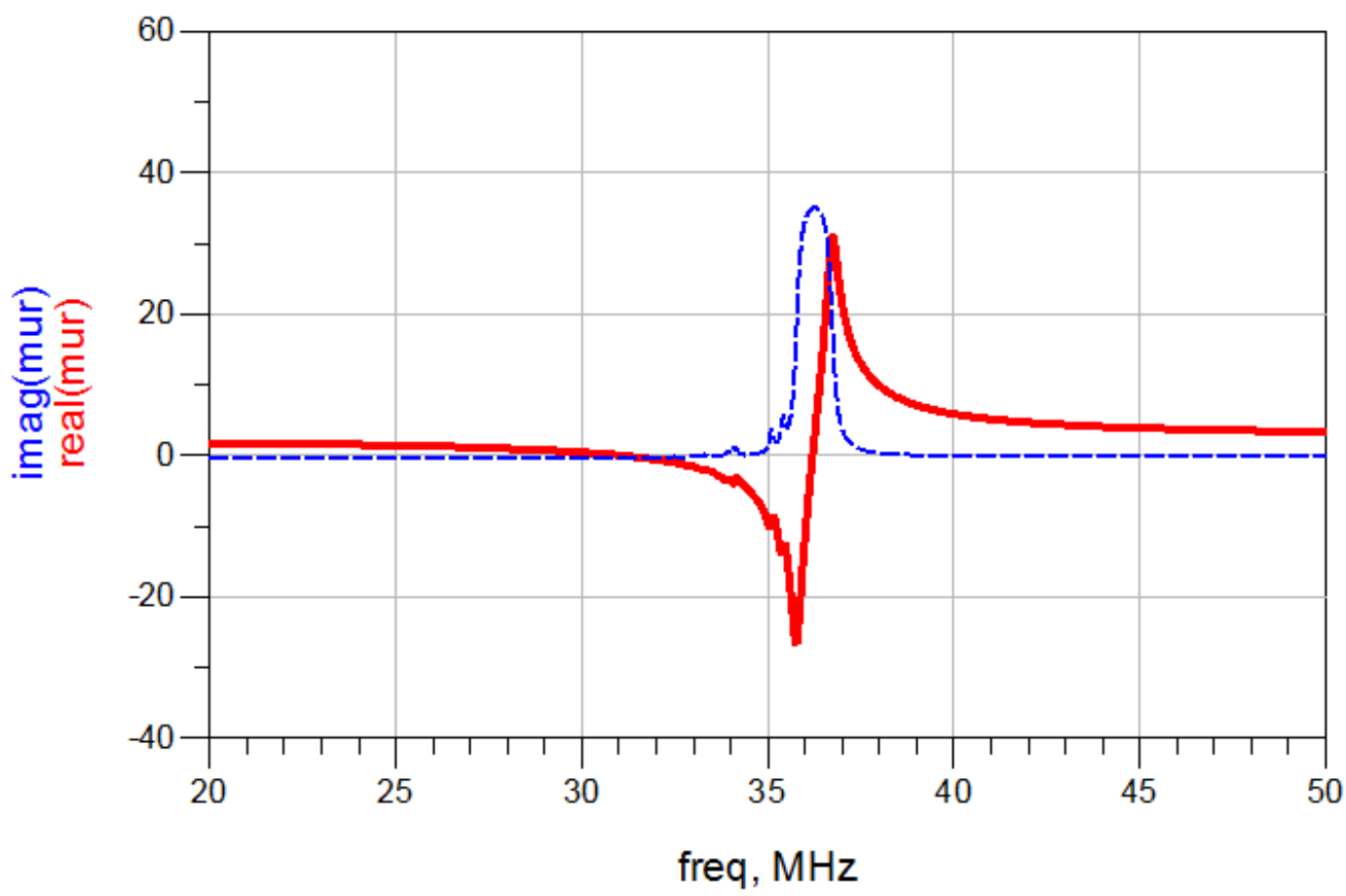

Figure 5.2: Numerical result for the real (red solid) and imaginary part (blue dash) of $\mu_{r}$ on

As it can be seen in Figure 5.2, the estimation of the resonance frequency given by the numerical simulation of the unit cell differs slightly from the analytical one (see Figure 4.14). The discrepancy is a consequence of all the simplifying hypotheses done in order to obtain a simpler model. Nonetheless, the overall behavior and the estimated values of the equivalent permeability of the MTM in both the analytical model and the simulation are still quite similar:

$$
\mu_{r, M T M_{\text {simulated }}}=-27.9-j 35
$$




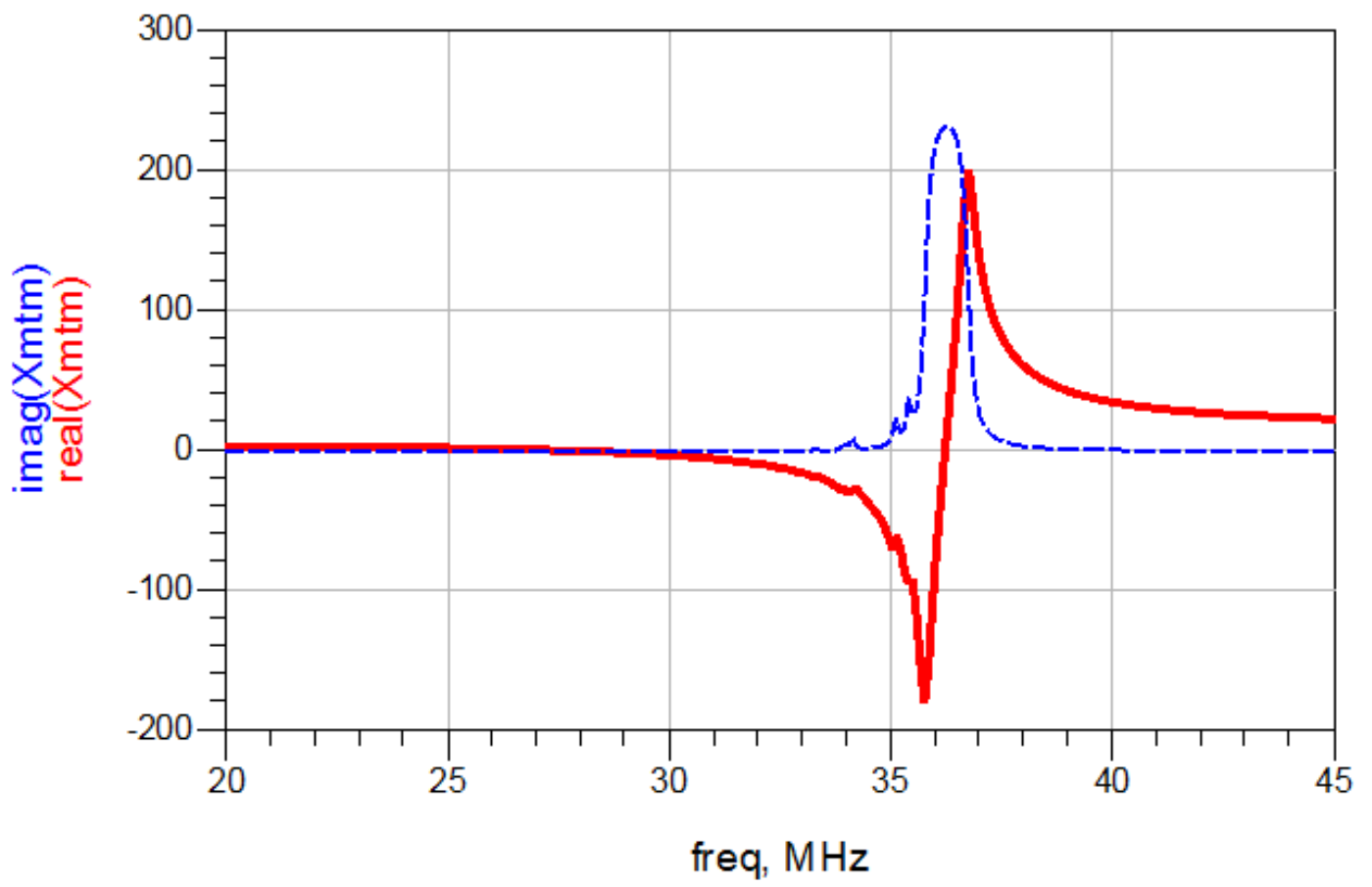

Figure 5.3: Numerical result for the real (red solid) and imaginary part (blue dash) of $X_{M T M}$ on ADS

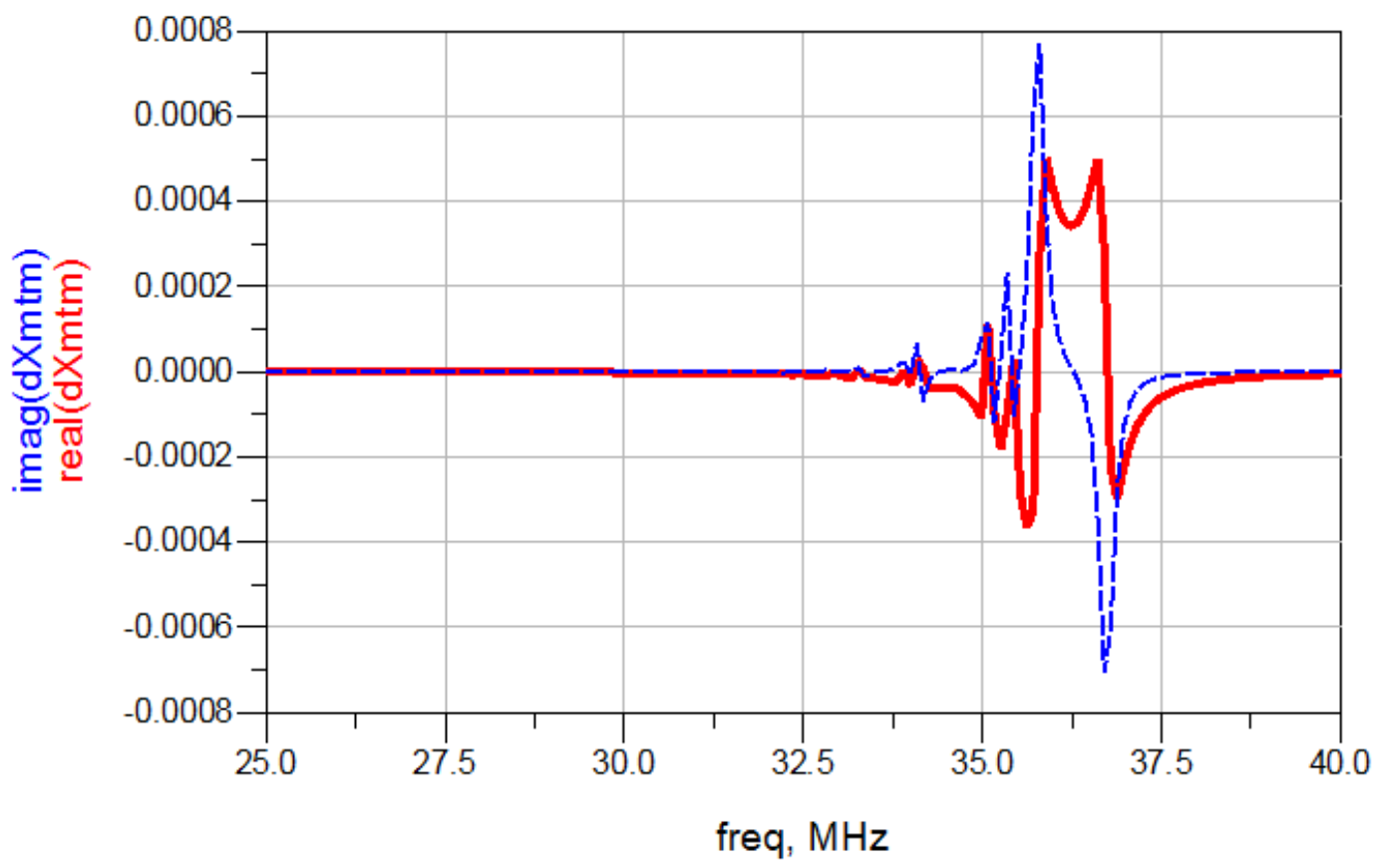

Figure 5.4: Numerical result for the real (red solid) and imaginary part (blue dash) of the first frequency derivative of $X_{M T M}$ on ADS 


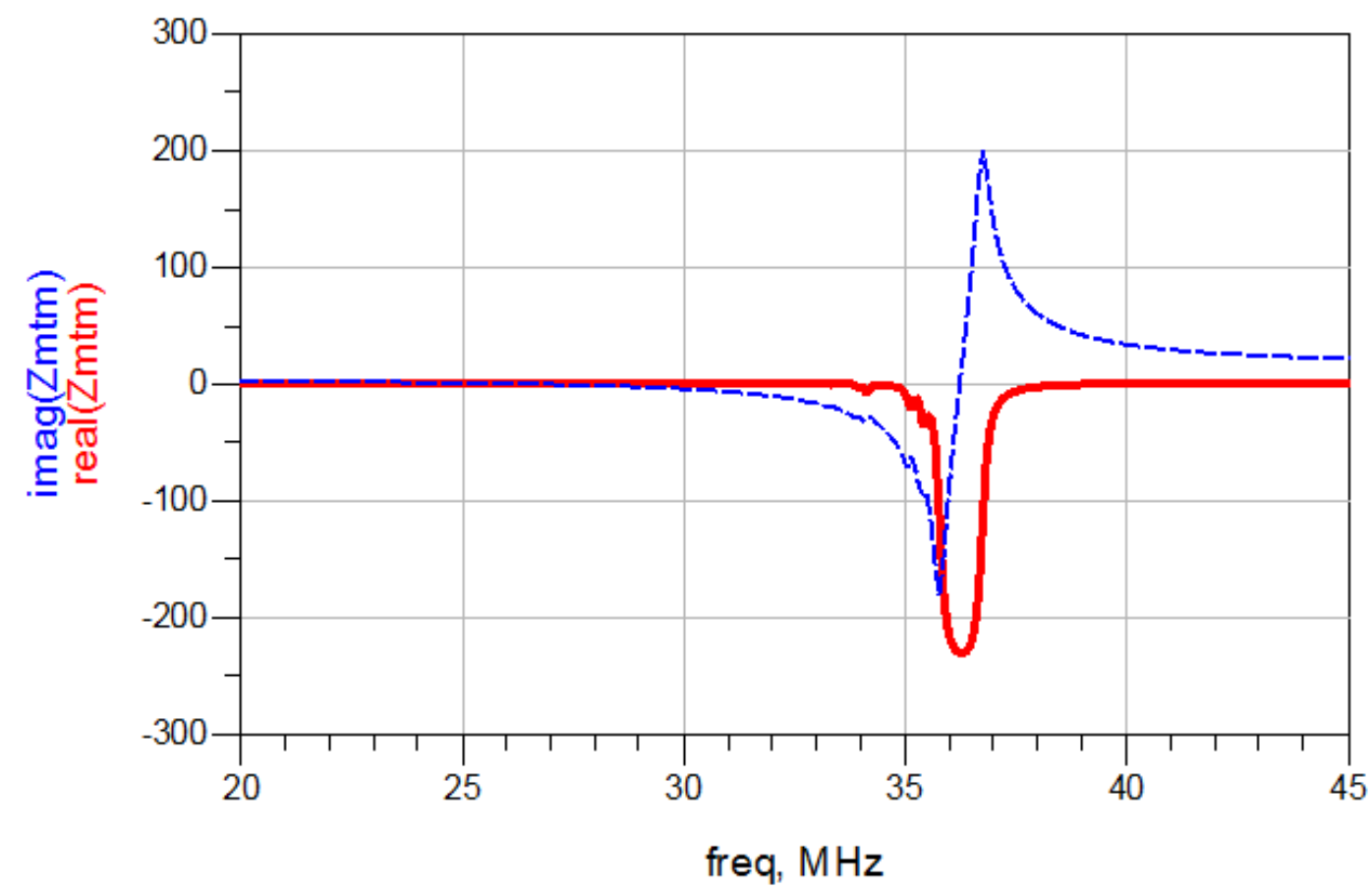

Figure 5.5: Numerical result for the real (red solid) and imaginary part (blue dash) of $Z_{M T M}$ on ADS

Also, as shown in Figure 5.3, Figure 5.4 and Figure 5.5, the numerical results for $X_{M T M}$, its derivative and $Z_{M T M}$ are in accordance with the analytical ones presented in Section 4.4 . 


\subsection{2.}

\section{Comparison between analytical and numerical results for the} proposed system without the metamaterial

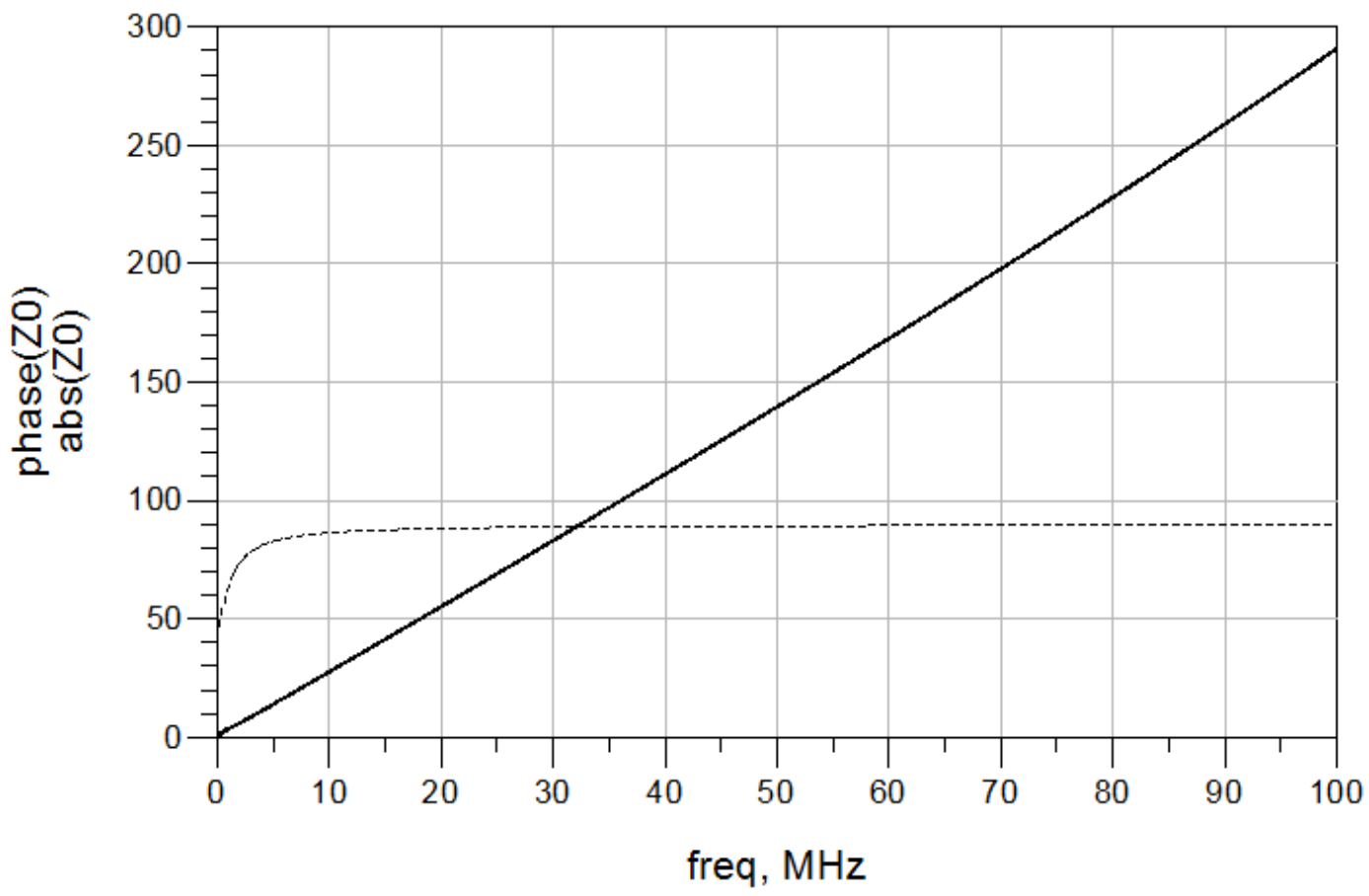

Figure 5.6: Analytical results for the magnitude (solid) and the phase (short dash) of $Z_{0}$ without the MTM slab

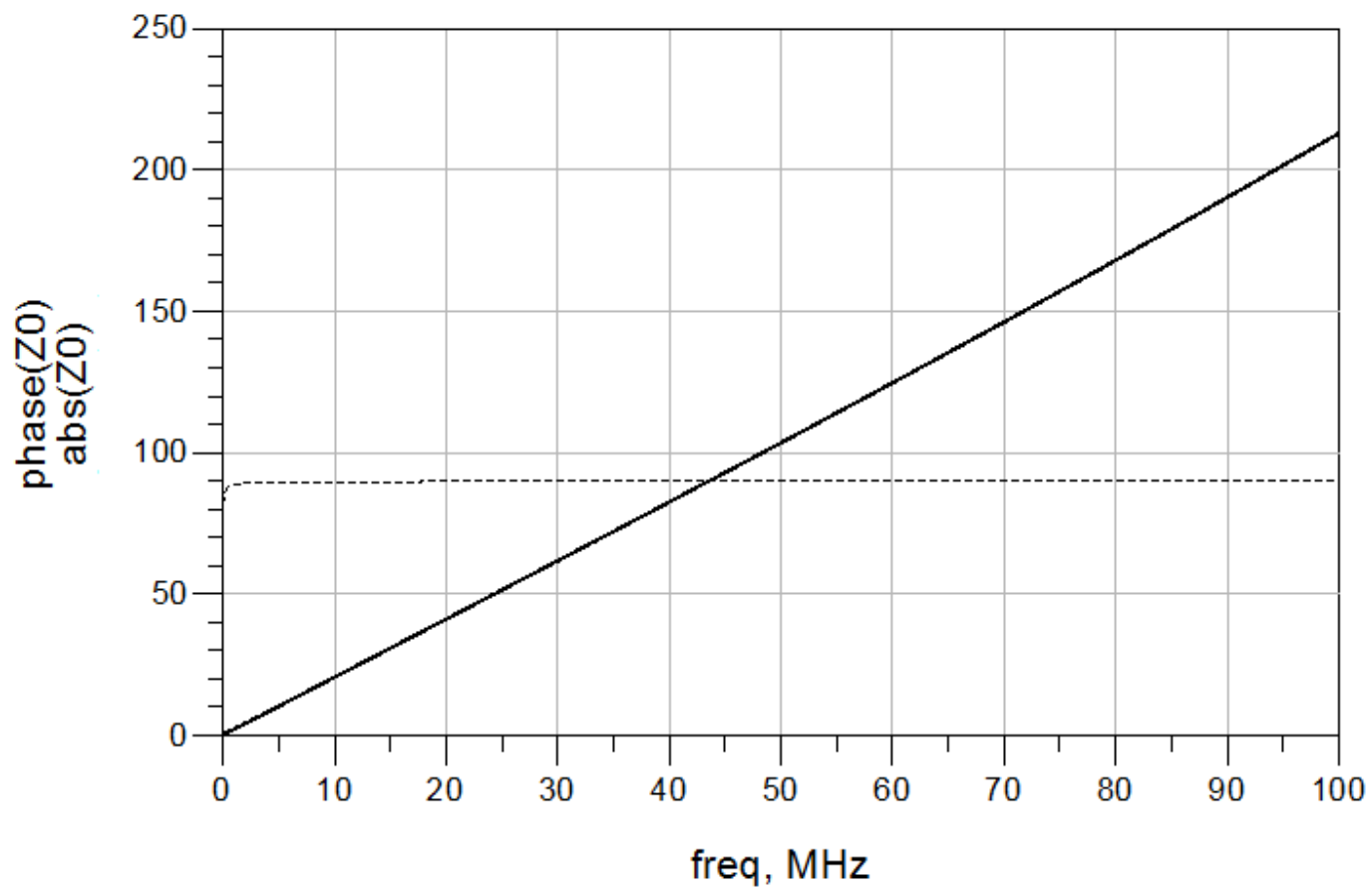

Figure 5.7: Numerical results for the magnitude (solid) and the phase (short dash) of $Z_{0}$ without the MTM slab 


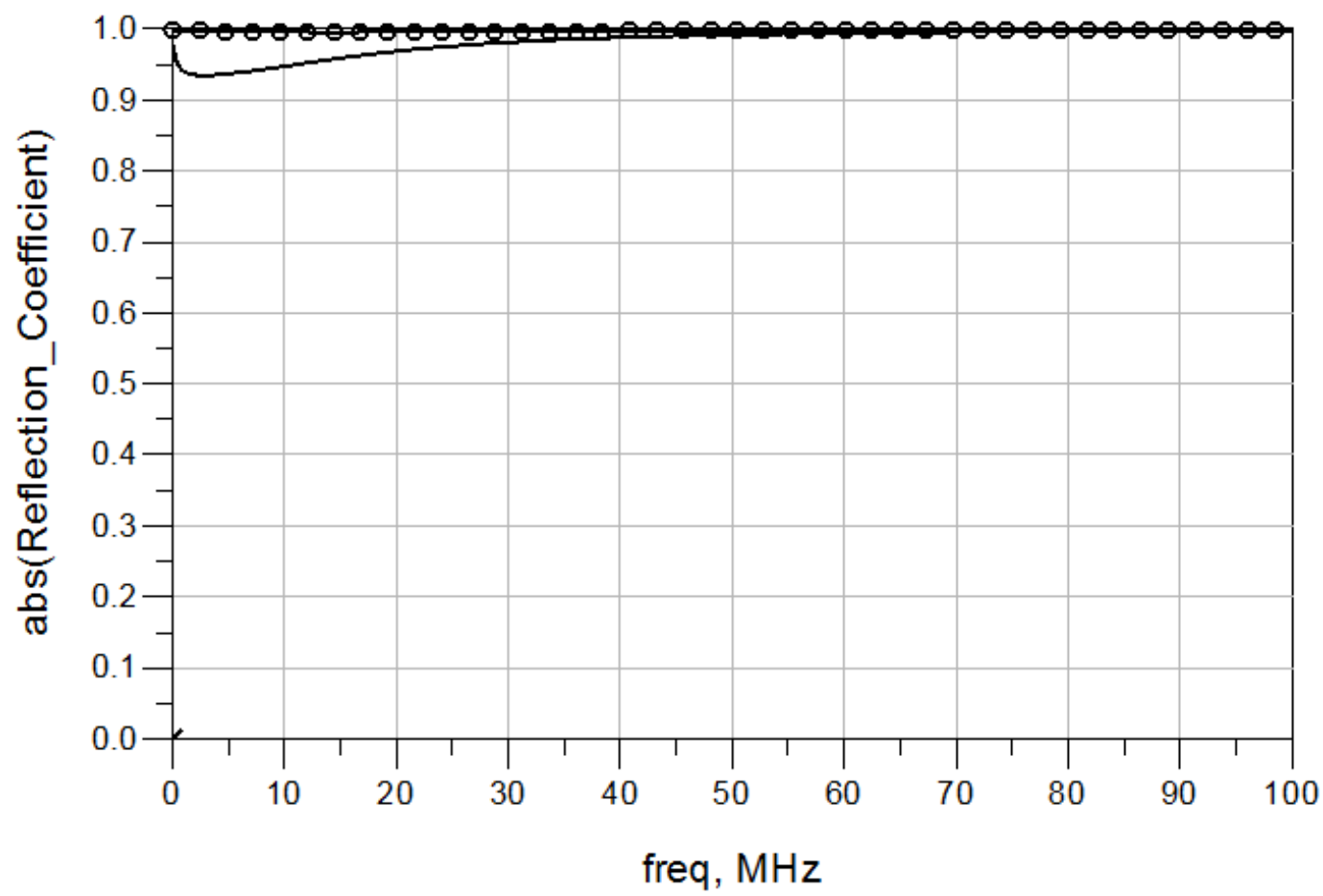

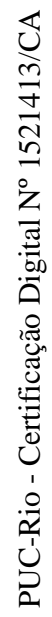

Figure 5.8: Analytical (solid line) and numerical (circle) results for the magnitude of $\Gamma$ without the MTM slab

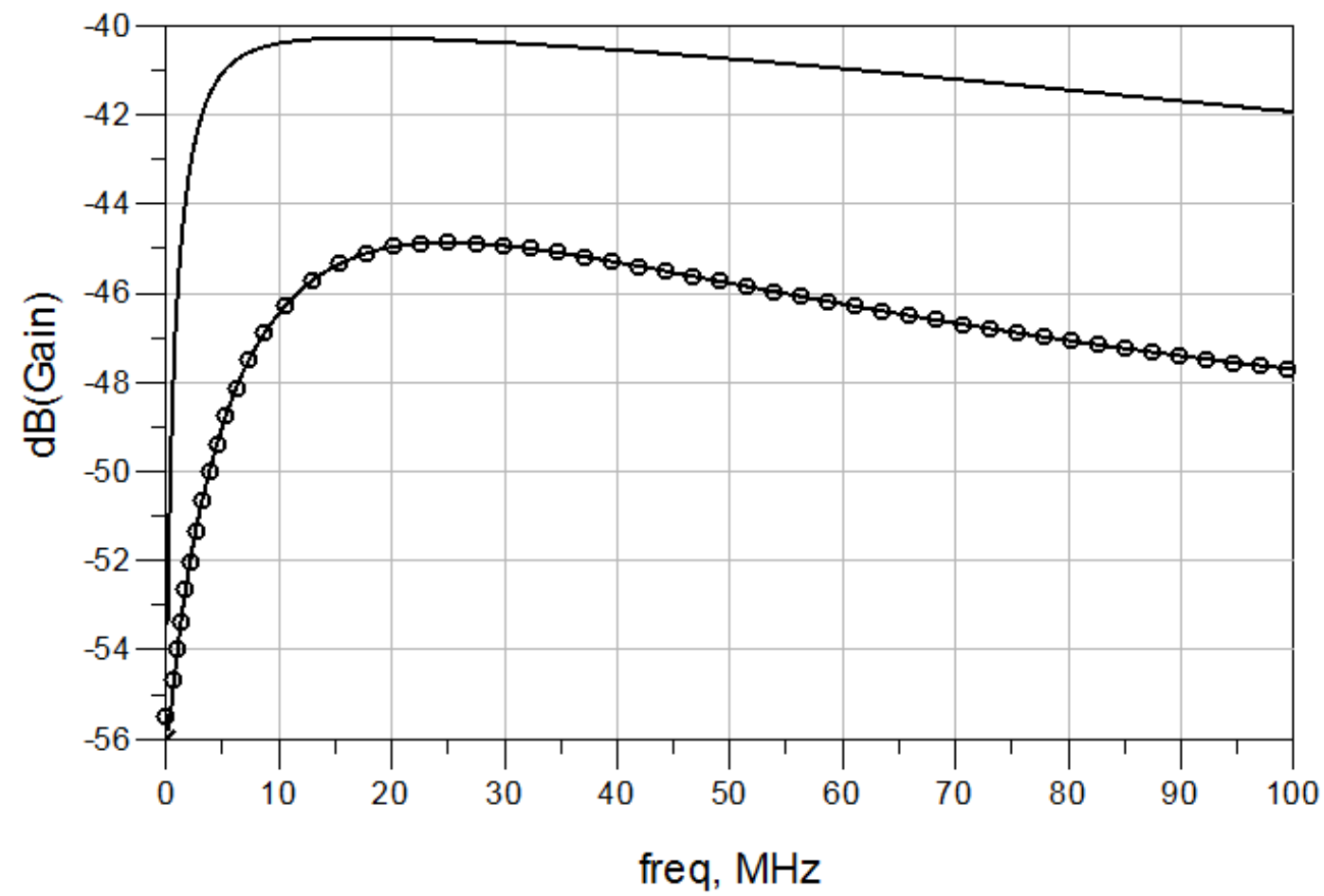

Figure 5.9: Analytical (solid line) and numerical (circle) results for the gain $G$ without the MTM slab 


\subsection{3.}

Comparison between analytical and numerical results for the proposed system with the metamaterial

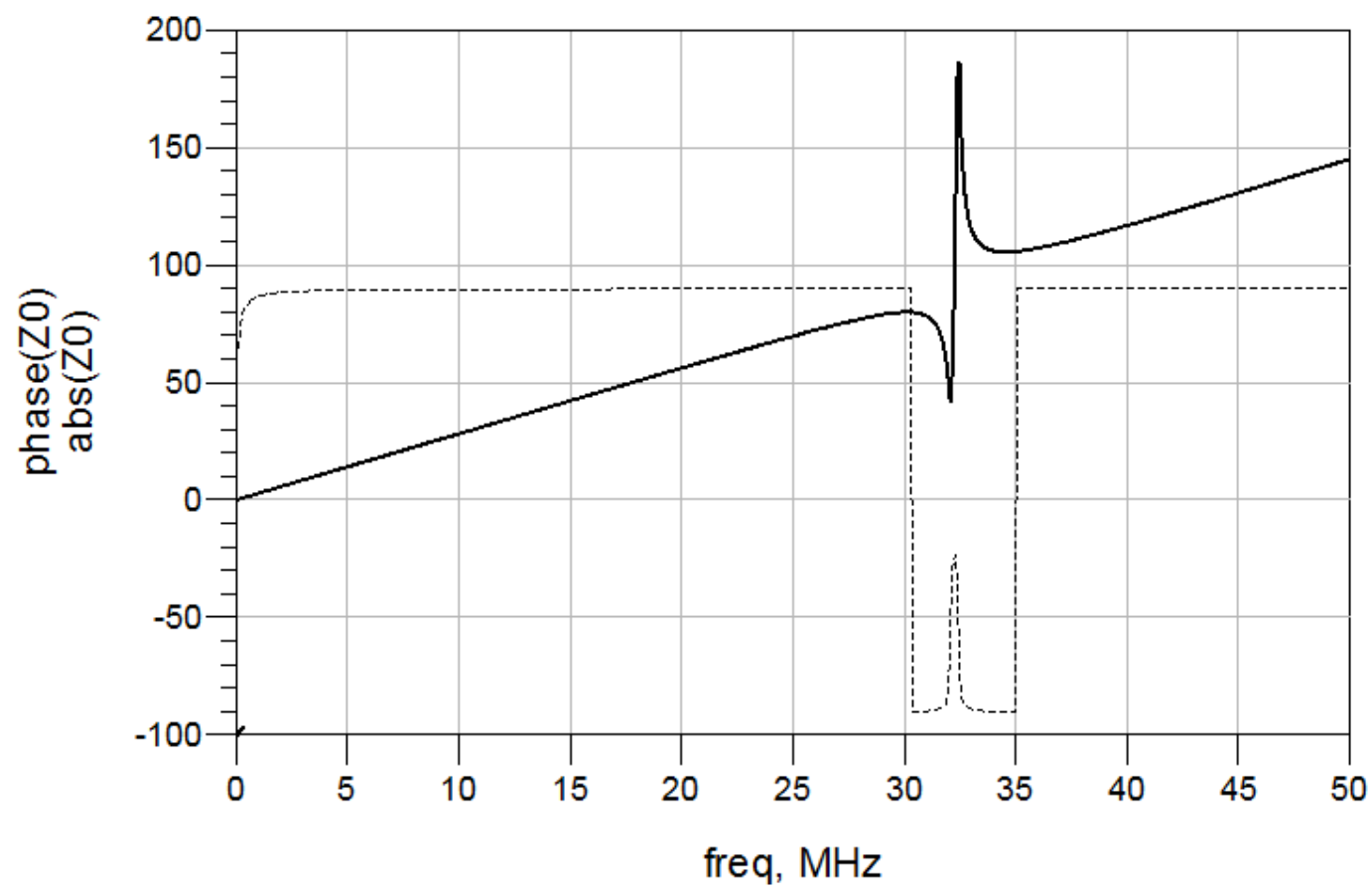

Figure 5.10: Analytical results for the magnitude (solid) and the phase (short dash) of $Z_{0}$ without the MTM slab

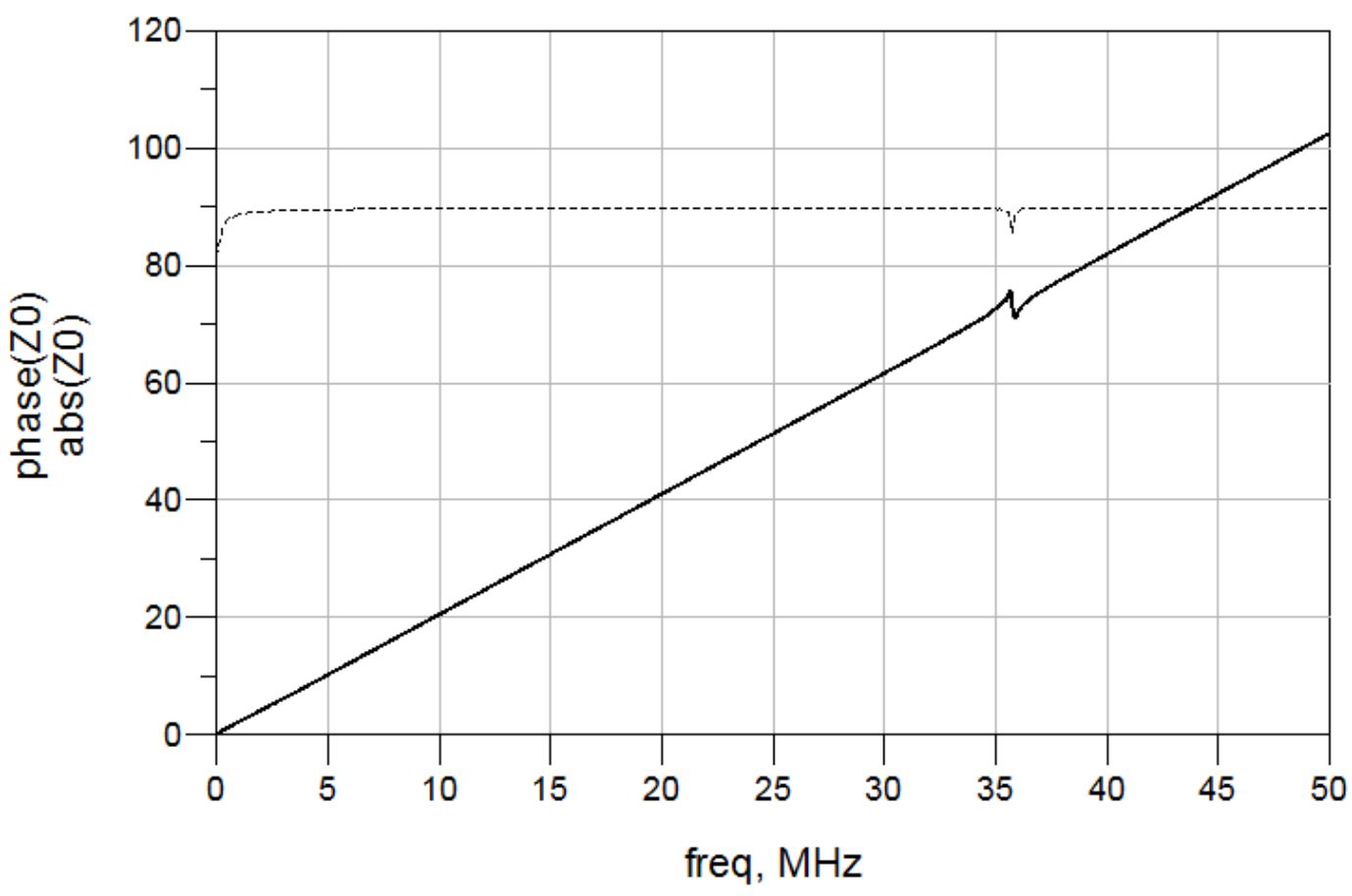

Figure 5.11: Numerical results for the magnitude (solid) and the phase (short dash) of $Z_{0}$ without the MTM slab 


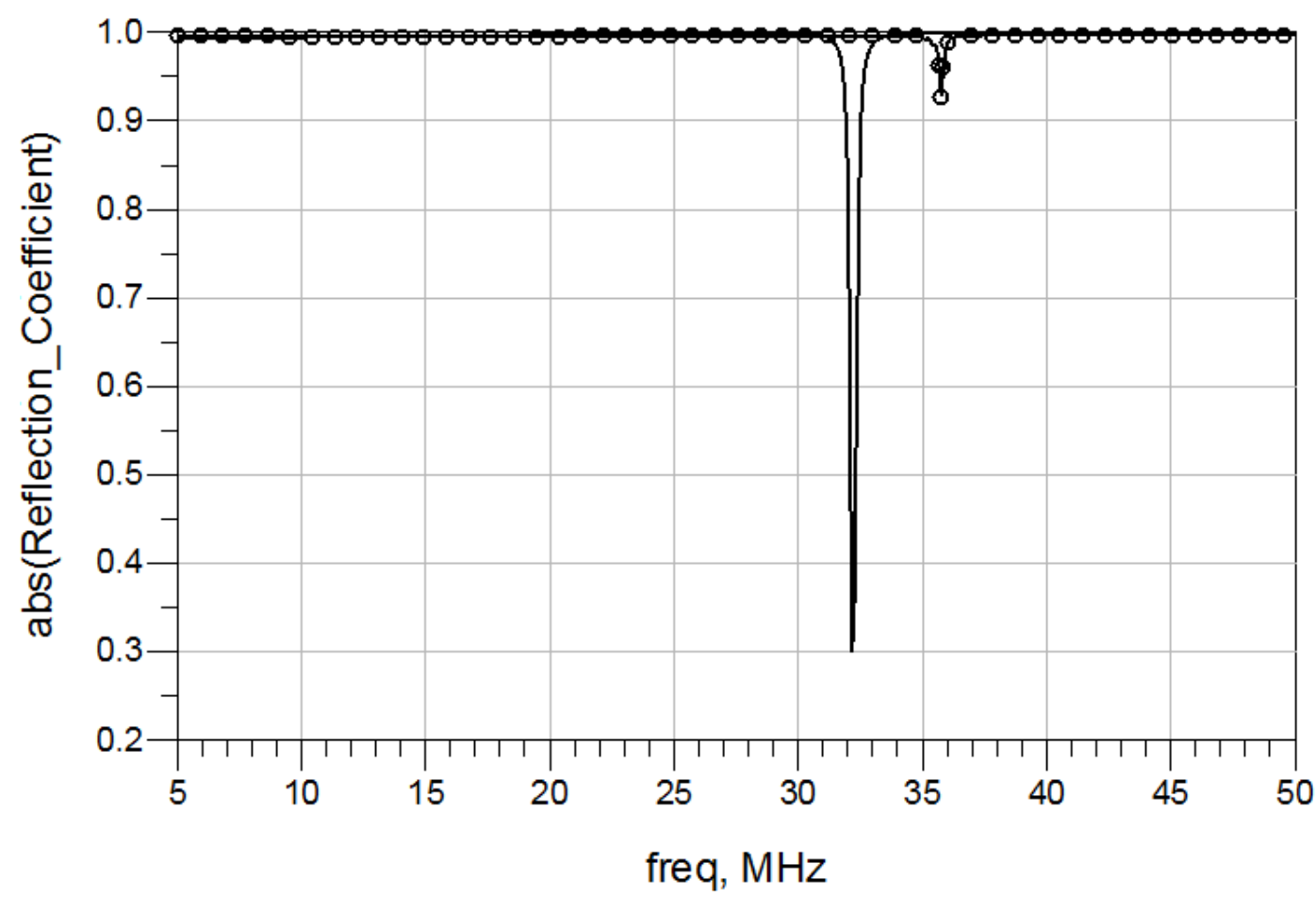

Figure 5.12: Analytical (solid line) and numerical (circle) results for the magnitude of $\Gamma$

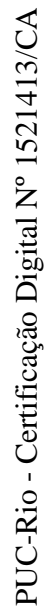
with the MTM slab

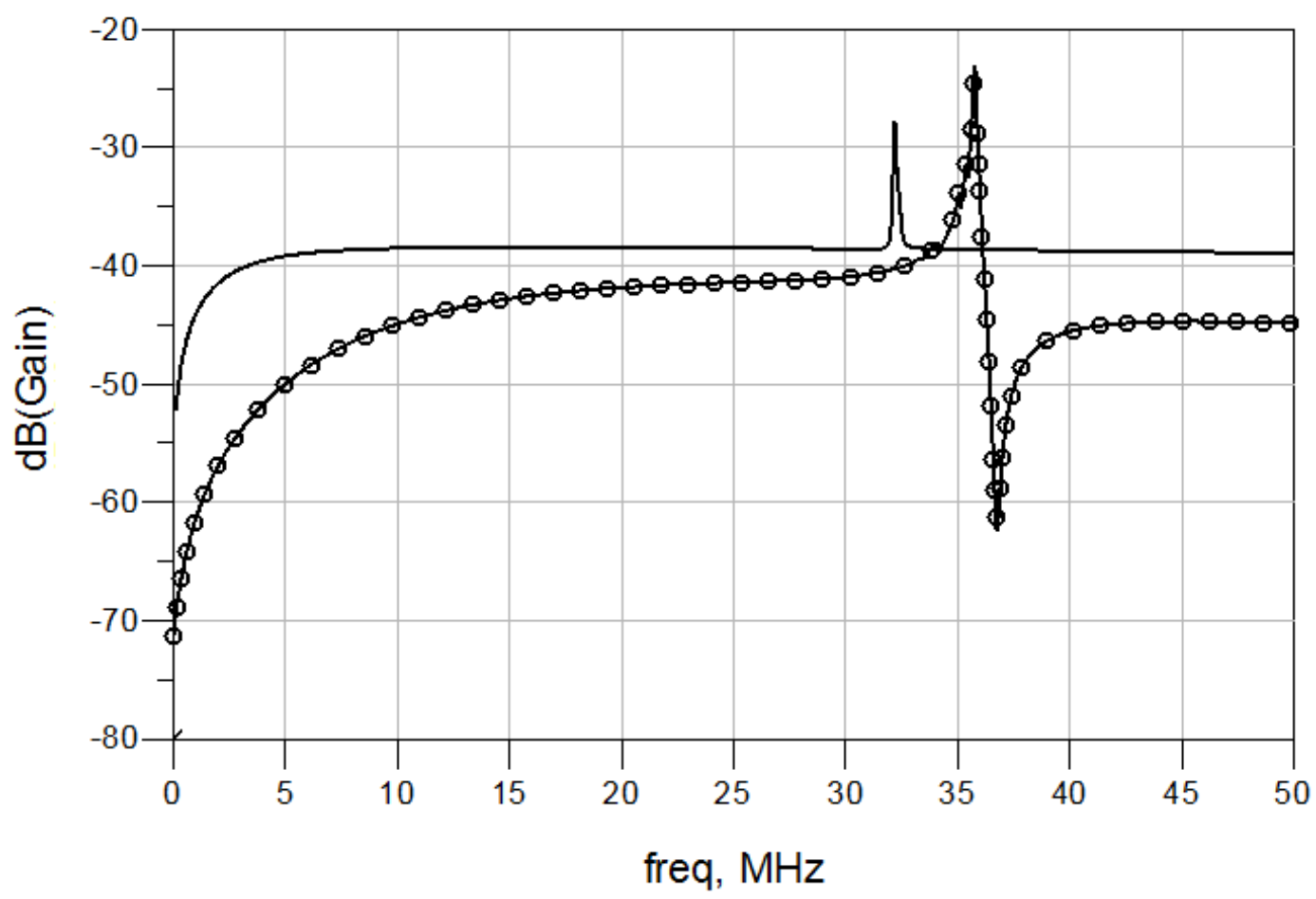

Figure 5.13: Analytical (solid line) and numerical (circle) results for the gain $G$ with the MTM slab 


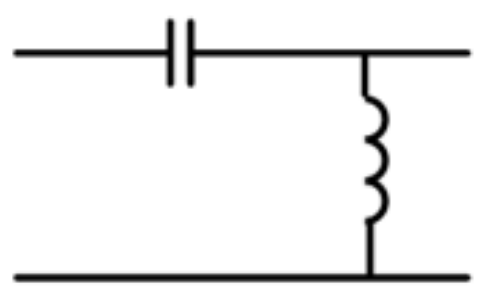

Figure 5.14: a MGTL is analogous to a high-pass filter

As shown in the graphs presented in Sections 5.2.2 and 5.2.3 (it is particularly clear in Figure 5.8), the analytical results diverge from the numerical results for low frequencies. This demonstrates that the coupling between two coils in free space behaves as a virtual TL only when the frequency is high enough. It is an expected result since MGTLs are analogous to high-pass filters (see Figure 5.14).

The difference in amplitude of the magnitude and the phase of $Z_{0}$ and the $\Gamma$ estimated by the analytical model and numerical simulation is due to the hypothesis made by the model of a spatially well-defined virtual magnetic potential $V_{m}$ for the $\mathrm{TL}$, which is not true in the considered system, considering that the potential varies randomly in space in freespace VMGTL case (a probabilistic model would be more suitable).

Nonetheless, despite the fact that this deterministic analytical model is not precise on its predictions, it still provides a good insight on the mechanisms governing the so-called MTM-enhanced coupling. It clearly demonstrates that the enhancement of the gain comes from the reduction of the reflection coefficient $\Gamma$ between the terminals. Since the MTM behaves as a negative impedance around the resonance, this behavior represents a sort of band-limited non-Foster impedance matching. 


\section{3.}

Simulations of the EM-field distribution for the proposed system

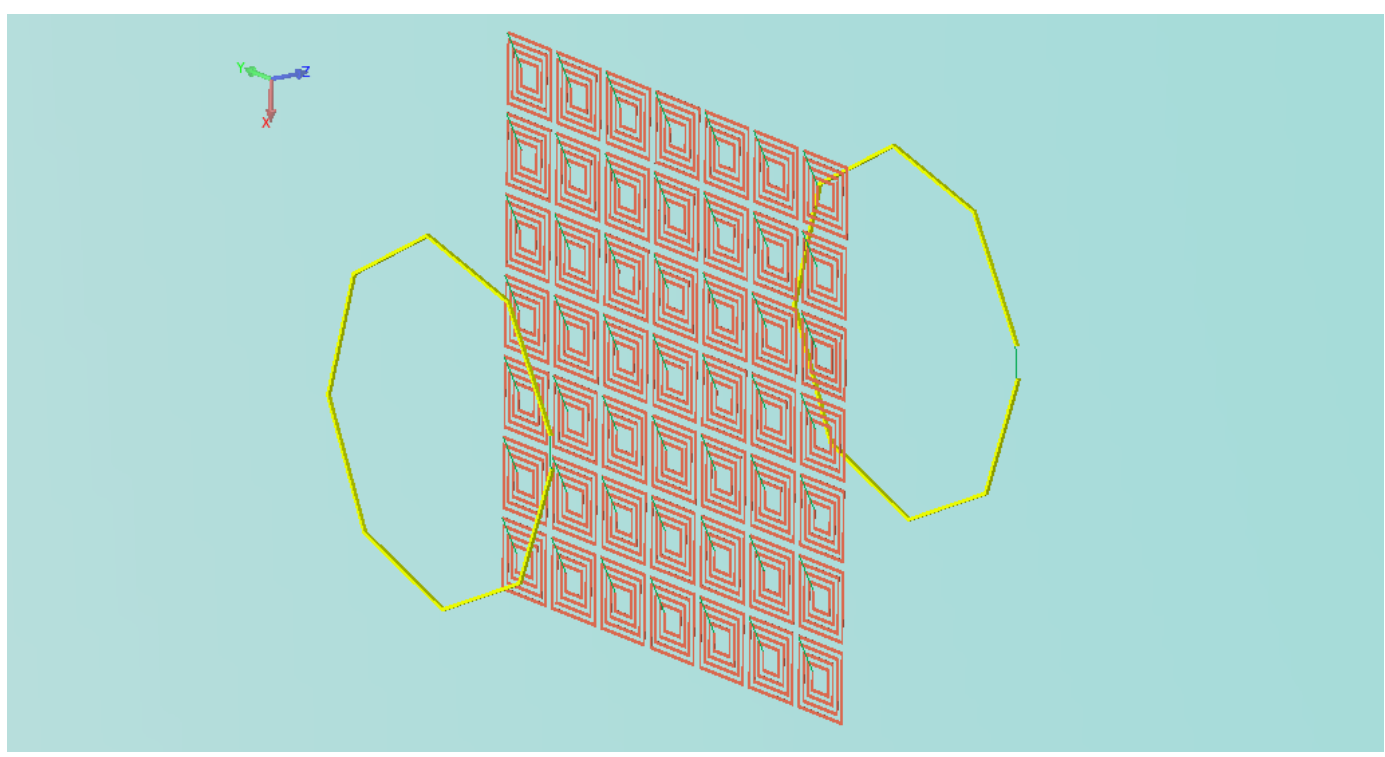

Figure 5.15: Simulation of the proposed system on EMPro

The simulated system on EMPro is shown in Figure 5.15.

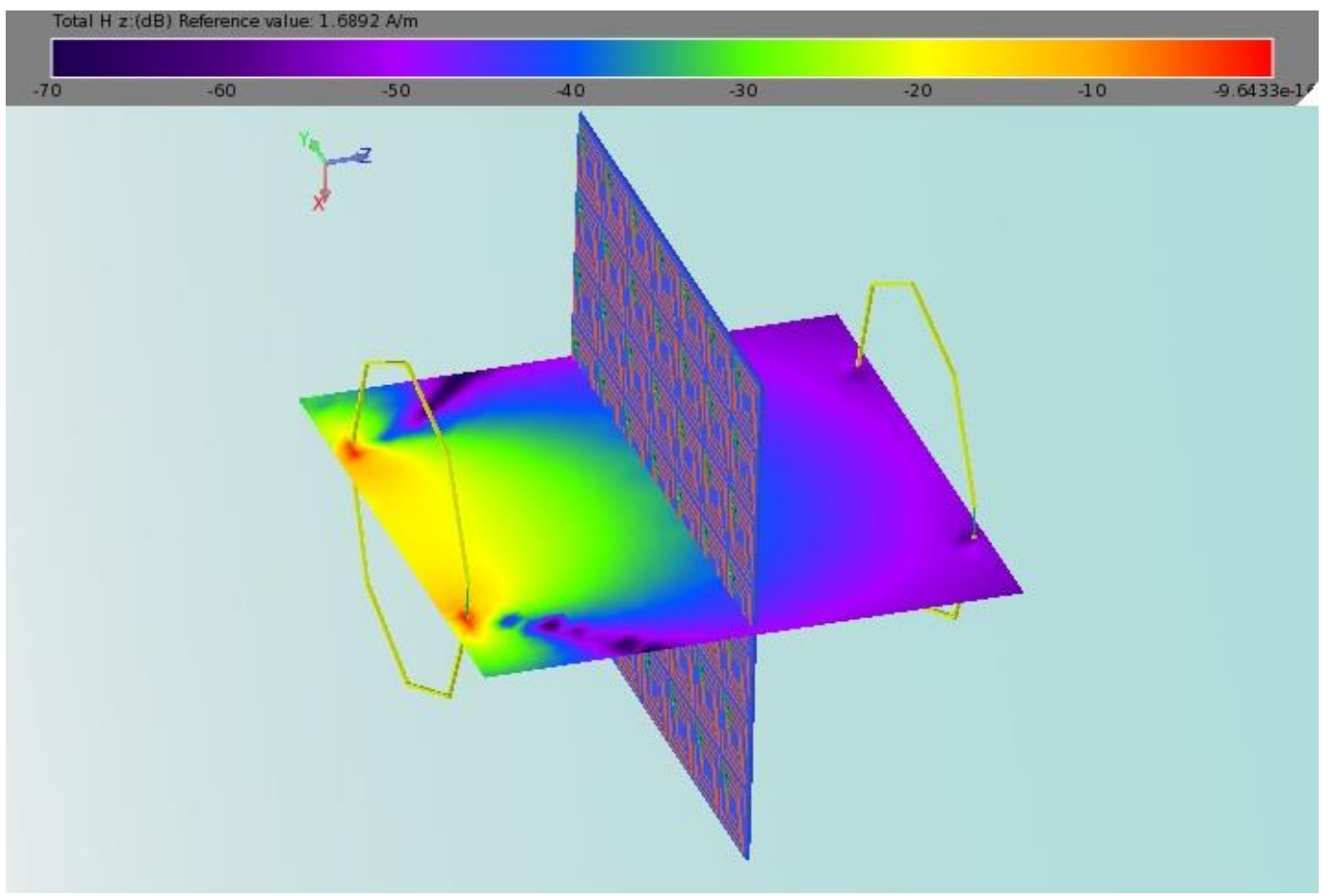

Figure 5.16: Magnitude of $H_{z}$ at the sub-resonance range at the plane $Y Z$ 


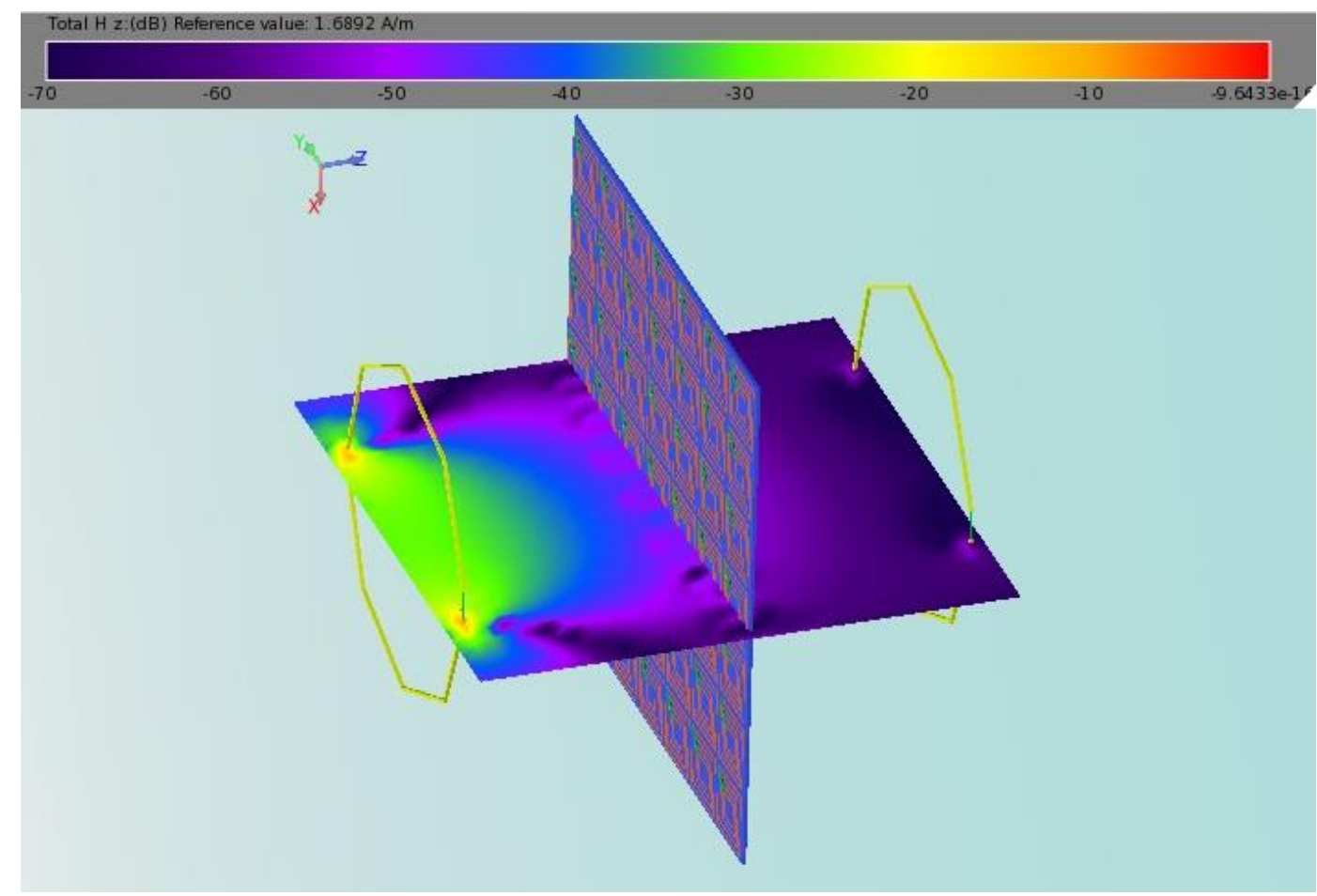

Figure 5.17: Magnitude of $H_{z}$ at the over-resonance range at the plane $Y Z$

U

As it was previously discussed, below the resonance the MTM is invisible to the system (see Figure 5.16) and above it behaves as a positive reactance (see Figure 5.17).

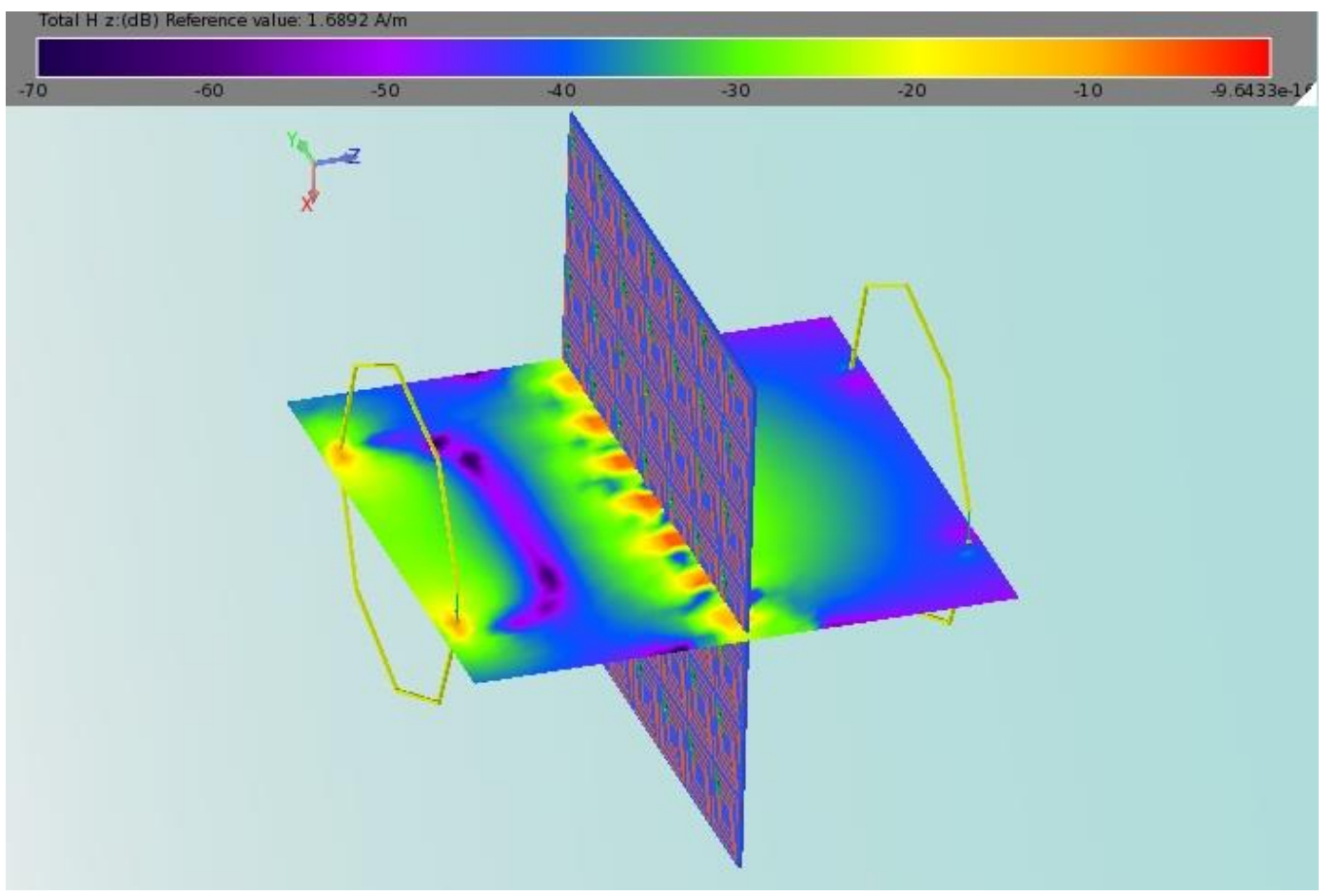

Figure 5.18: Magnitude of $H_{z}$ at the resonance at the plane $Y Z$ 
When the MTM is at its operating frequency $\omega_{0}$, it presents the highest magnetic flux density in the system and is the main source of magnetic potential for the magnetic link (see Figure 5.18).

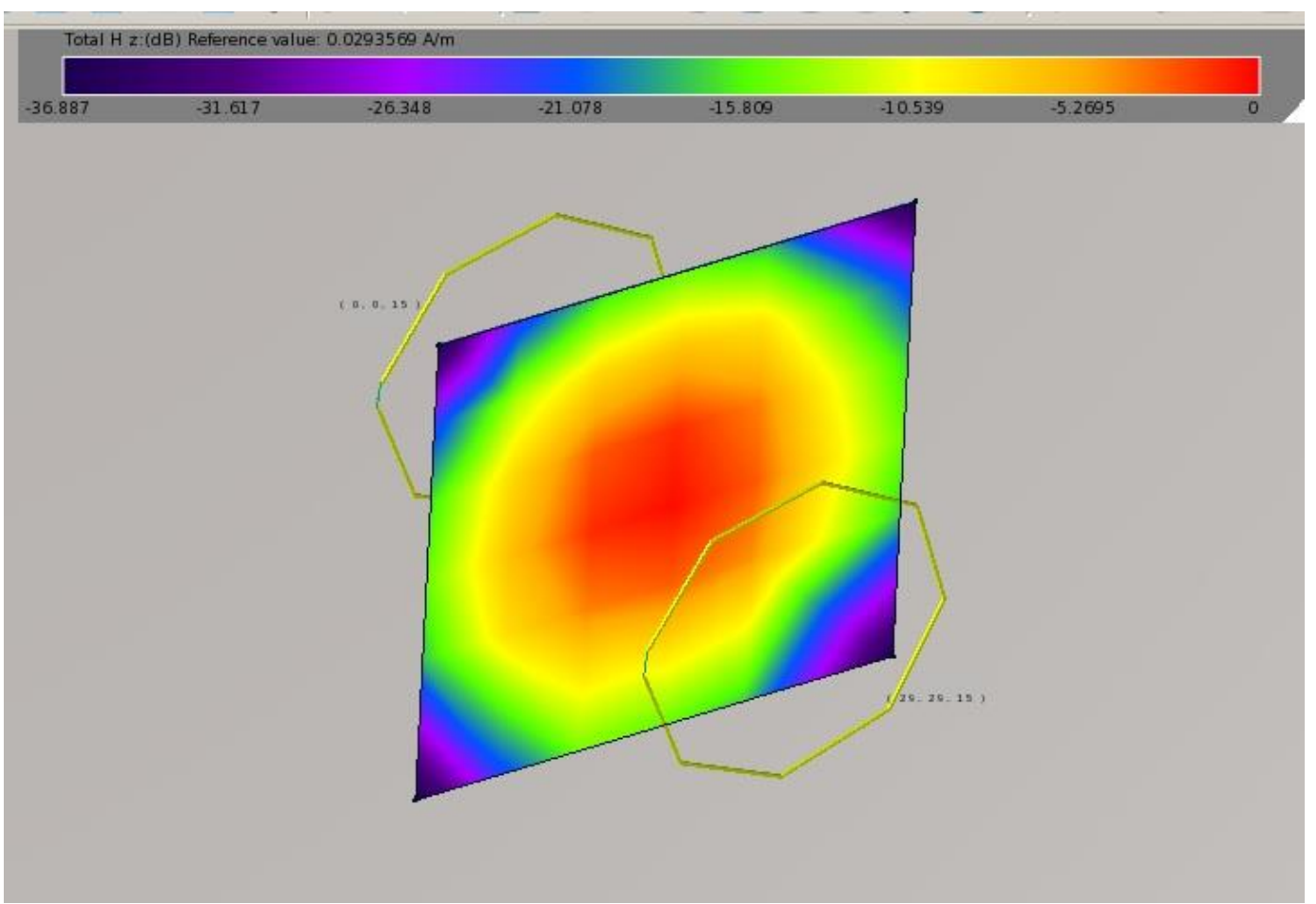

Figure 5.19: Magnitude of $H_{z}$ at the sub-resonance range at the MTM surface

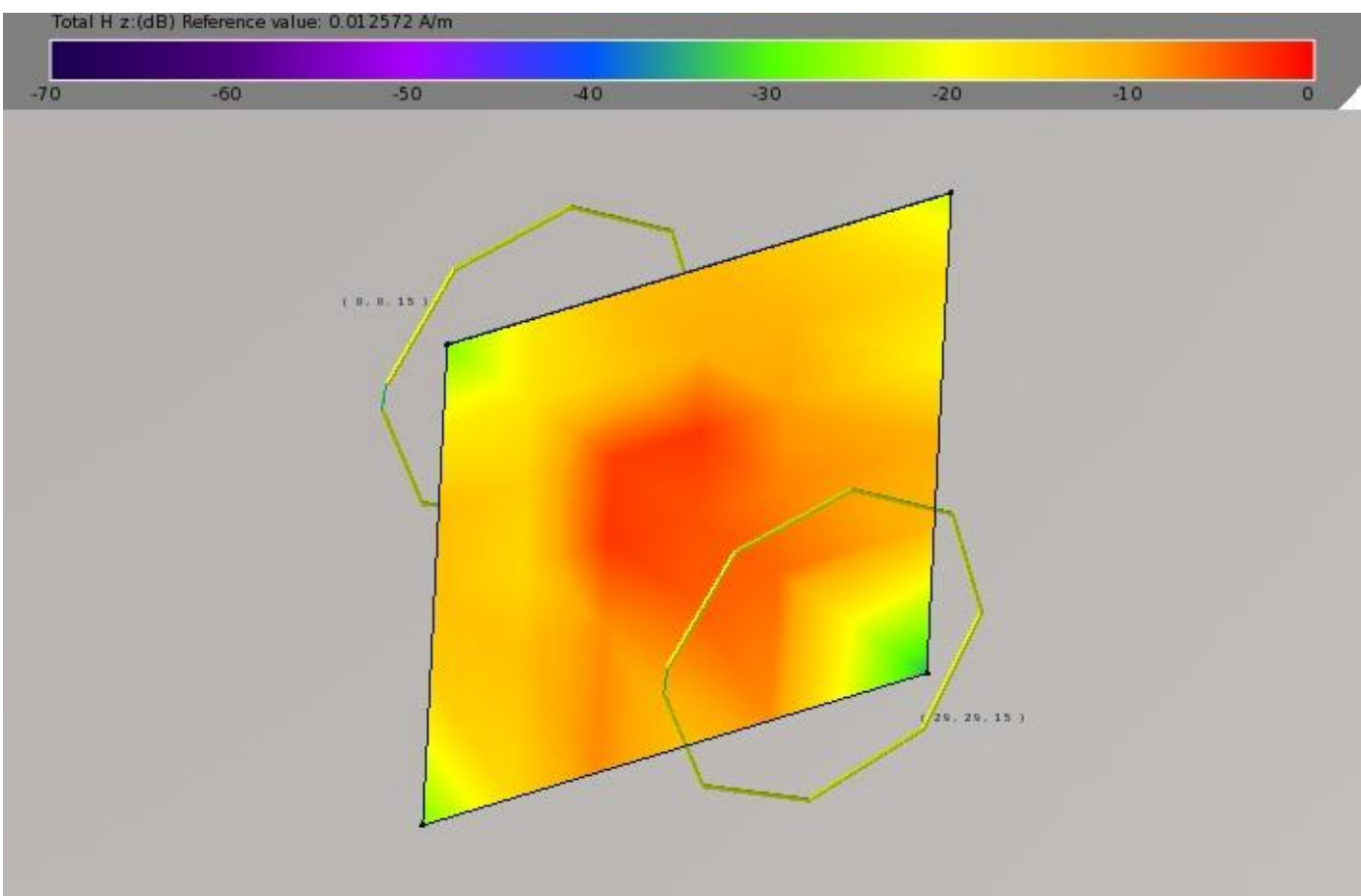

Figure 5.20: Magnitude of $H_{z}$ at the over-resonance range at the MTM surface 


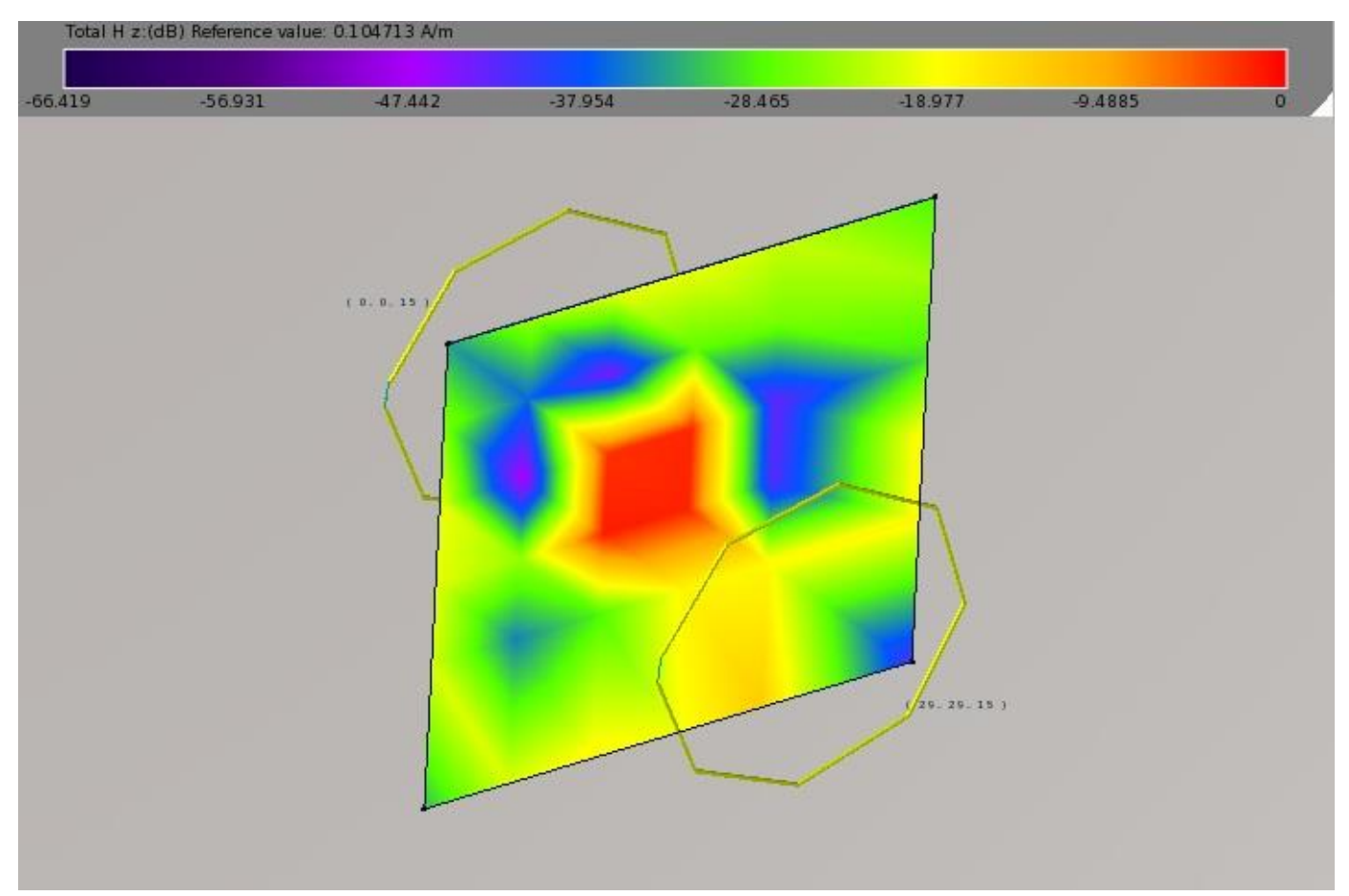

Figure 5.21: Magnitude of $H_{z}$ at the resonance at the MTM surface

By comparing the simulations presented on Figure 5.19, Figure 5.20 and Figure 5.21, it can be seen that $H_{z}$ is focalized close to the center of the surface of the MTM at the resonance, which implies the focalization of the magnetic flux in this area as a consequence (see Sections 2.2 and 2.6). 


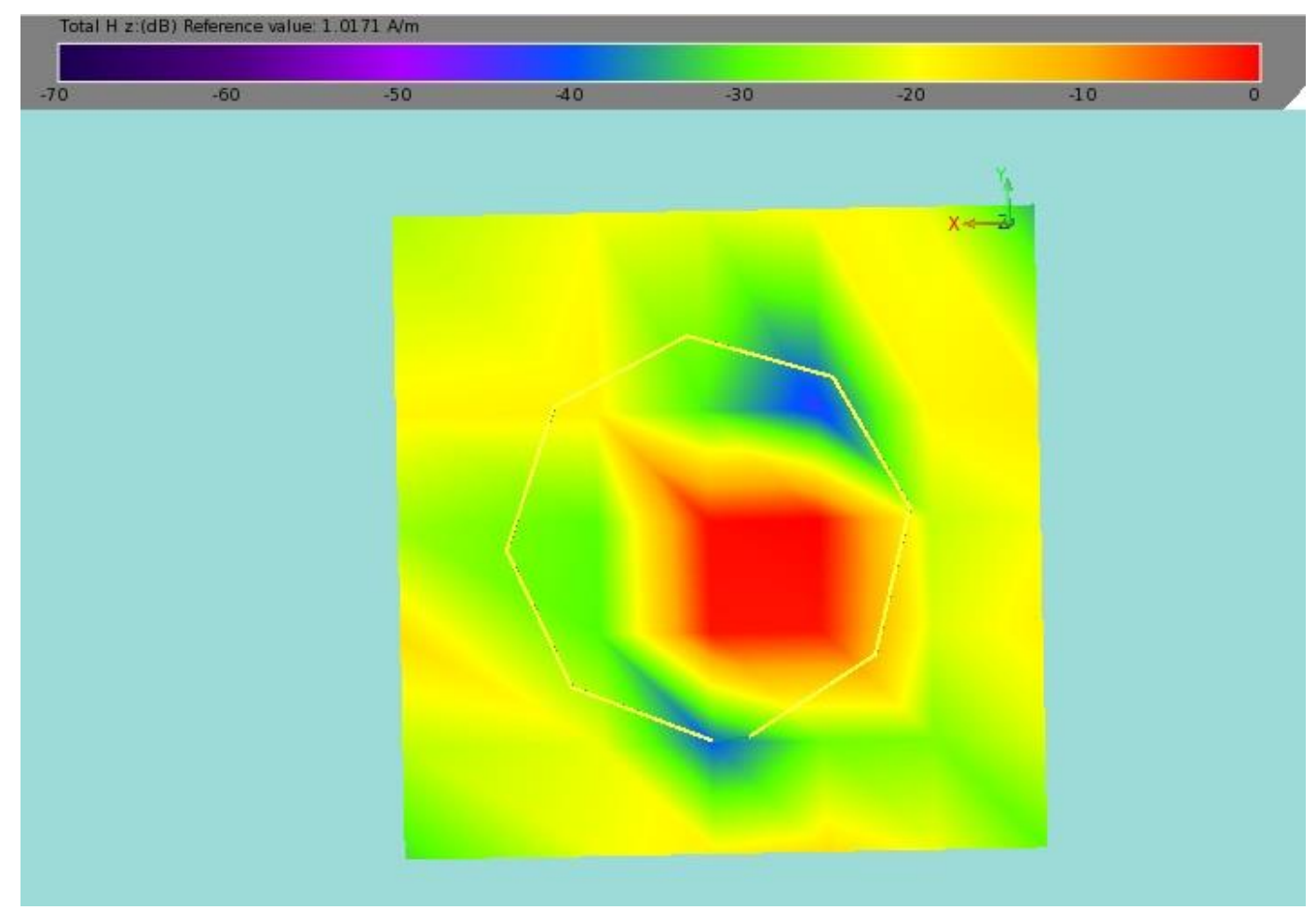

Figure 5.22: Focalization of $H_{z}$ is deviated from the center due to material losses

U

Due to material losses $\left(\mu^{\prime \prime} \neq 0\right)$, which puts the magnetic dipoles out of phase, the focus is deviated from the center (see Figure 5.22).

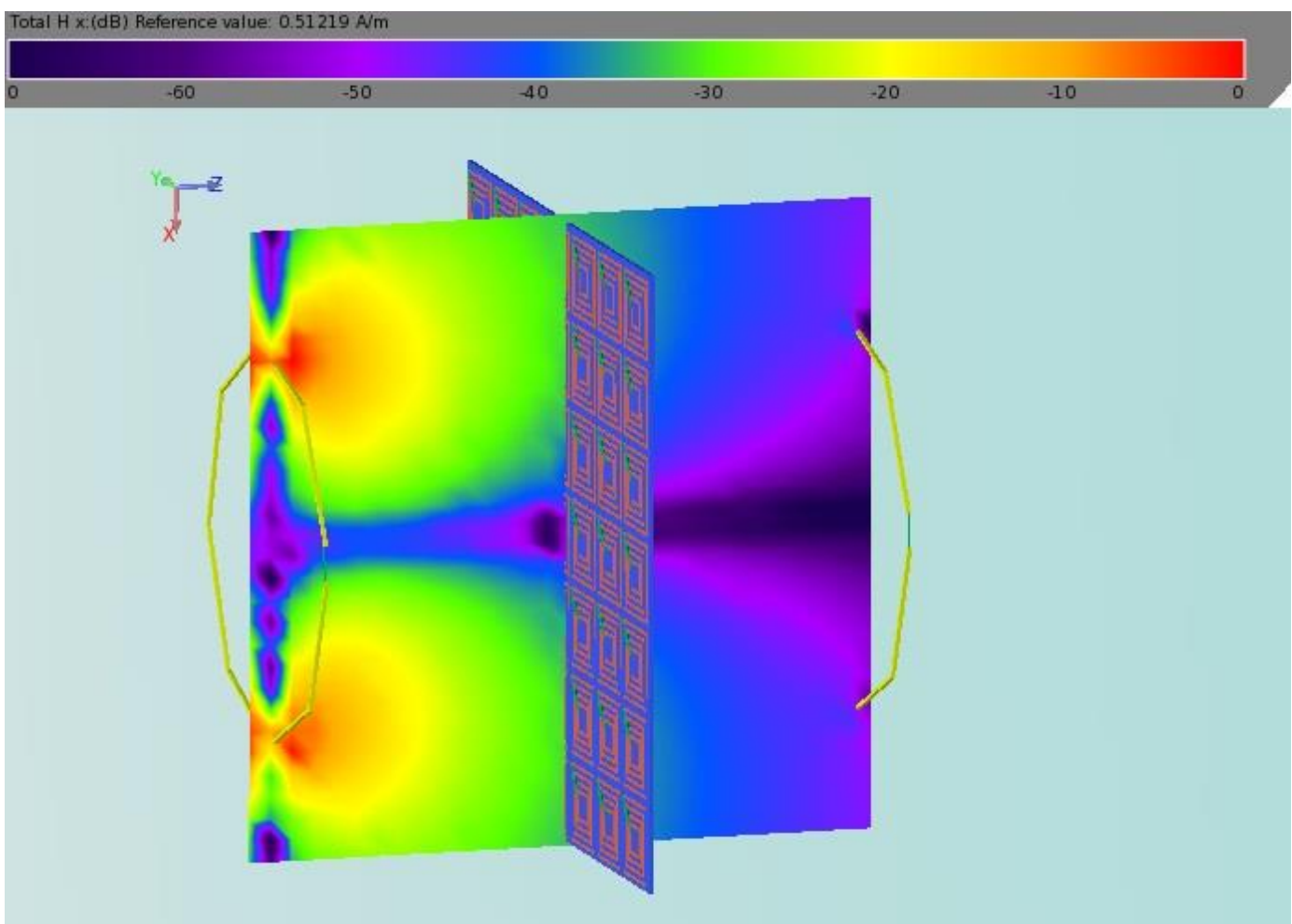

Figure 5.23: Magnitude of $H_{x}$ at the sub-resonance range at the plane $\mathrm{XZ}$ 


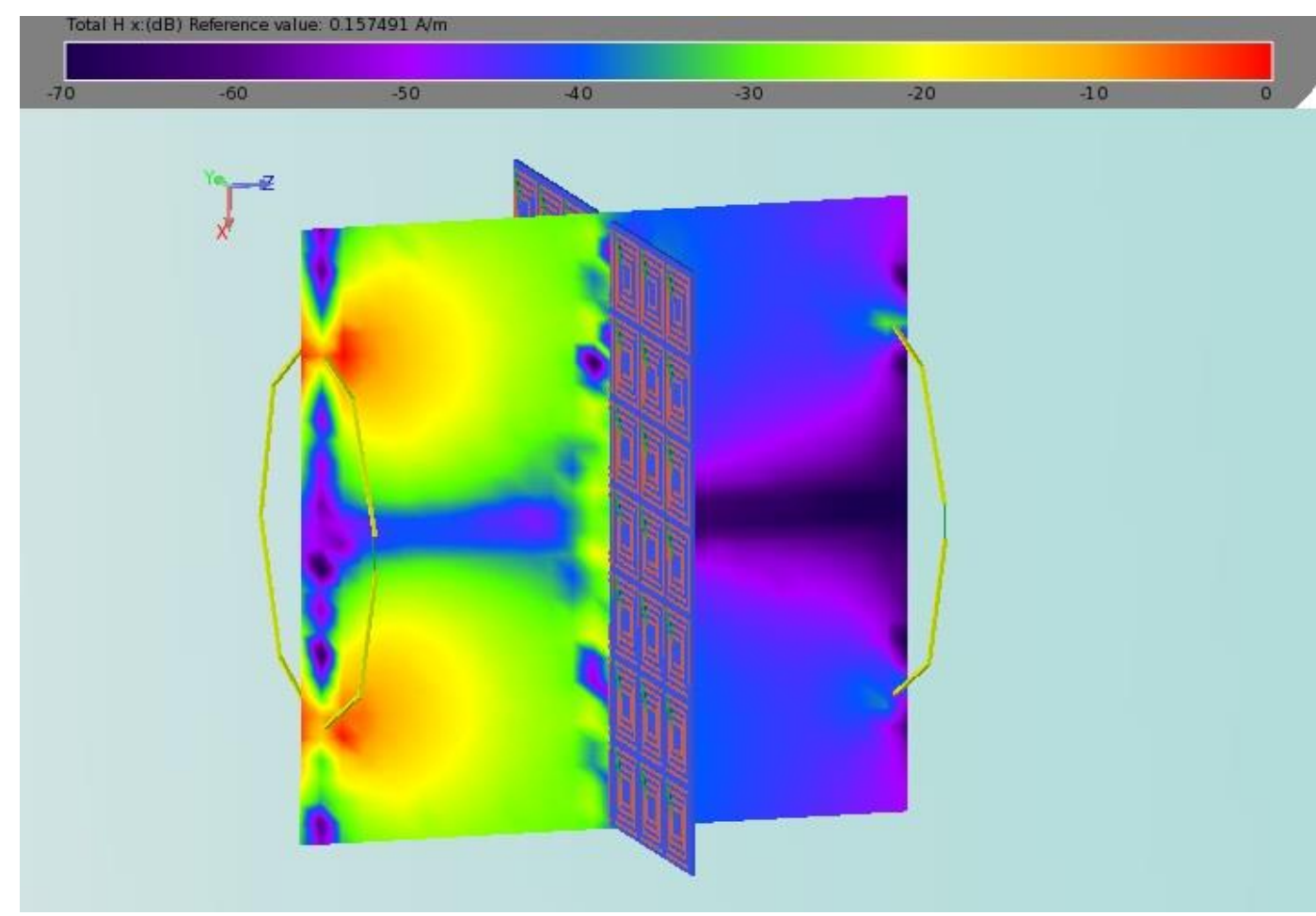

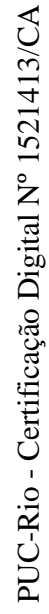

Figure 5.24: Magnitude of $H_{x}$ at the over-resonance range at the plane $\mathrm{XZ}$

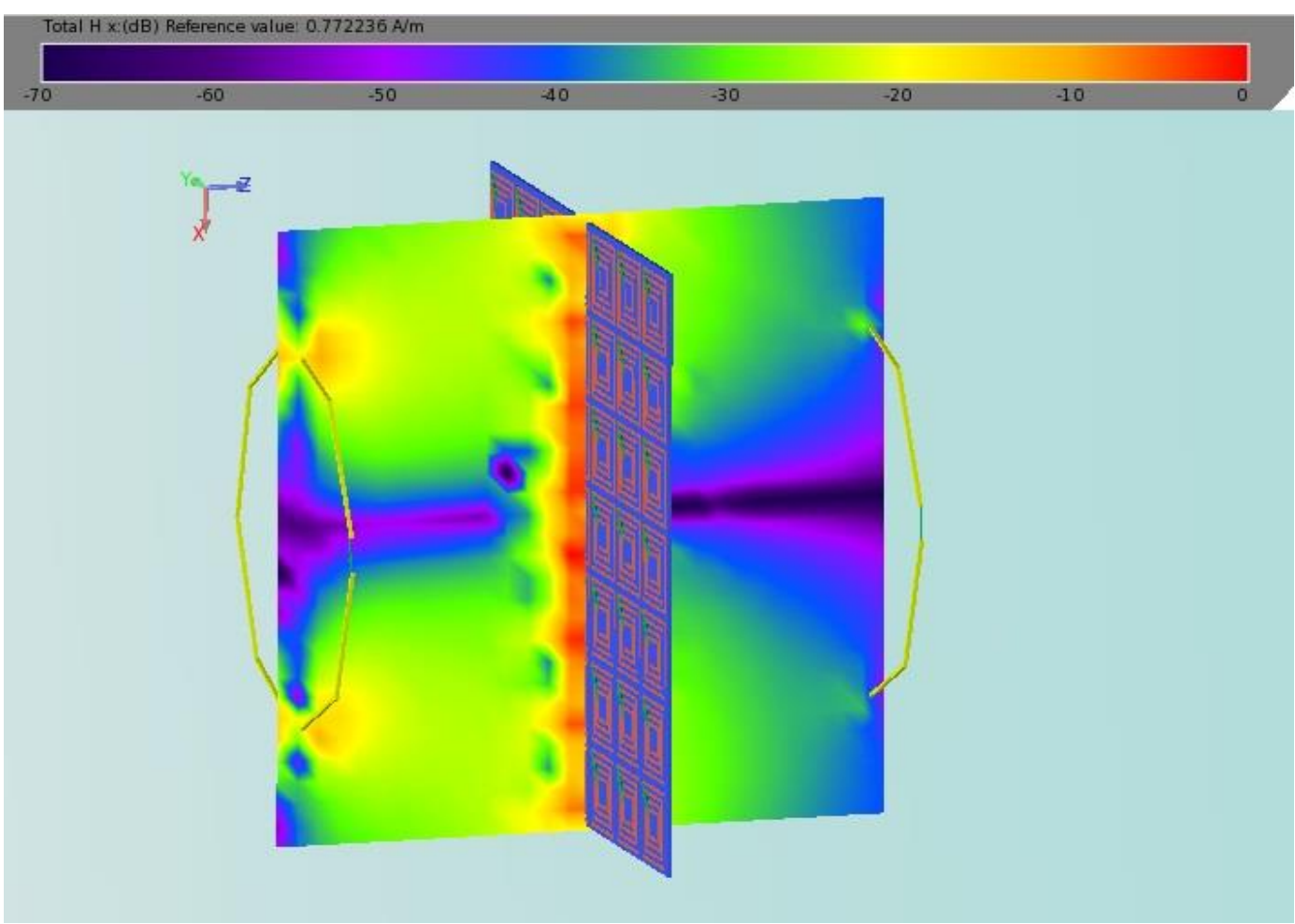

Figure 5.25: Magnitude of $H_{x}$ at the resonance at the plane XZ

The component $H_{x}$ of the magnetic field is the magnetic field stored in the virtual line formed between the drivers as a consequence of the magnetic 
potential difference between the incoming and the ongoing magnetic flux (See Figure 5.23 and Figure 5.24). As it is clearly shown in Figure 5.25, the magnetic potential $V_{m}$ is enhanced along the line when the MTM is activated and the system behaves more similarly to a confined VMGTL.

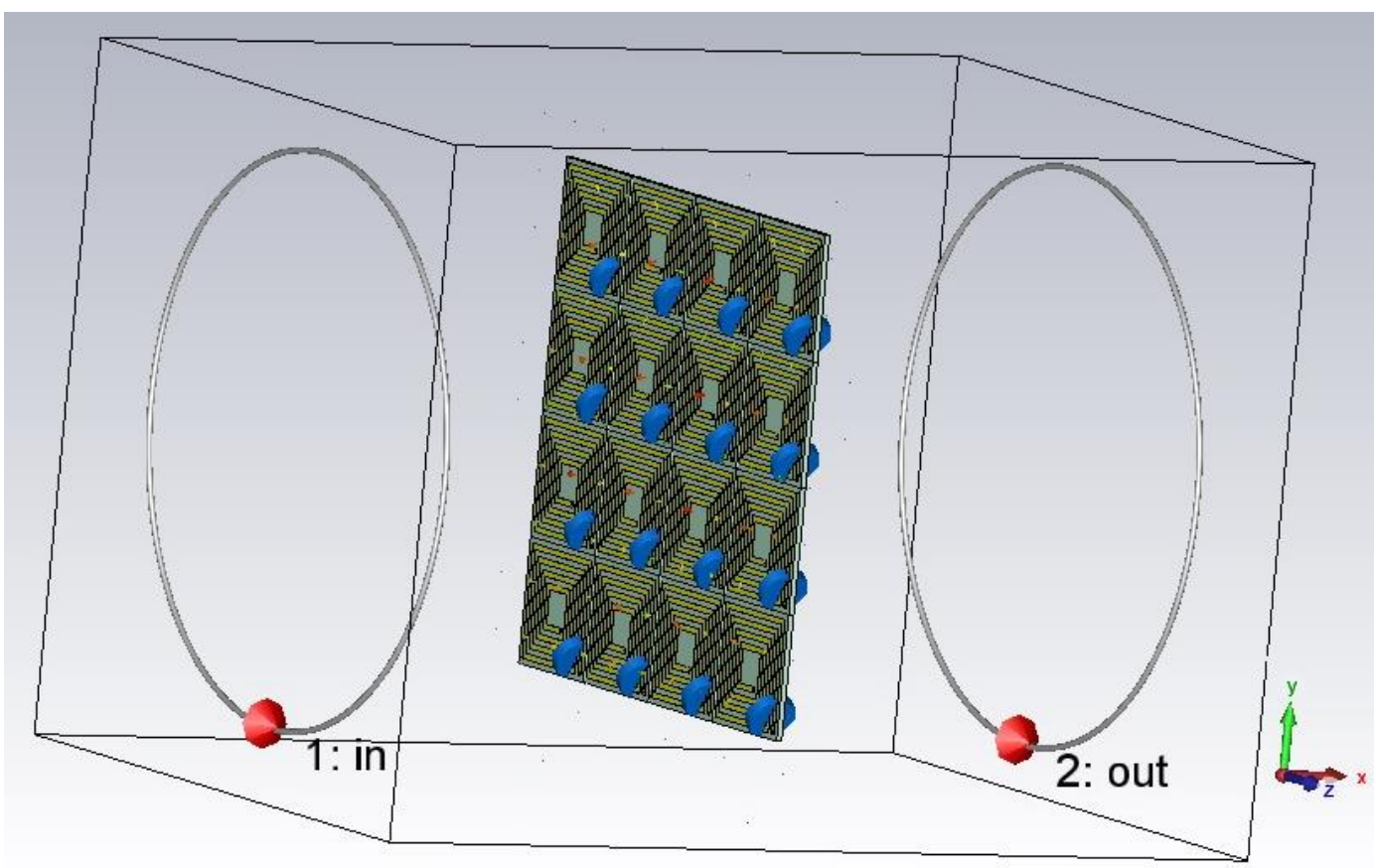

Figure 5.26: Simulation of the proposed system on CST Studio

In order to demonstrated the existence of a $\mu$-negative behavior around the resonance are a third simulation using the CST studio is presented, as shown in Figure 5.26.

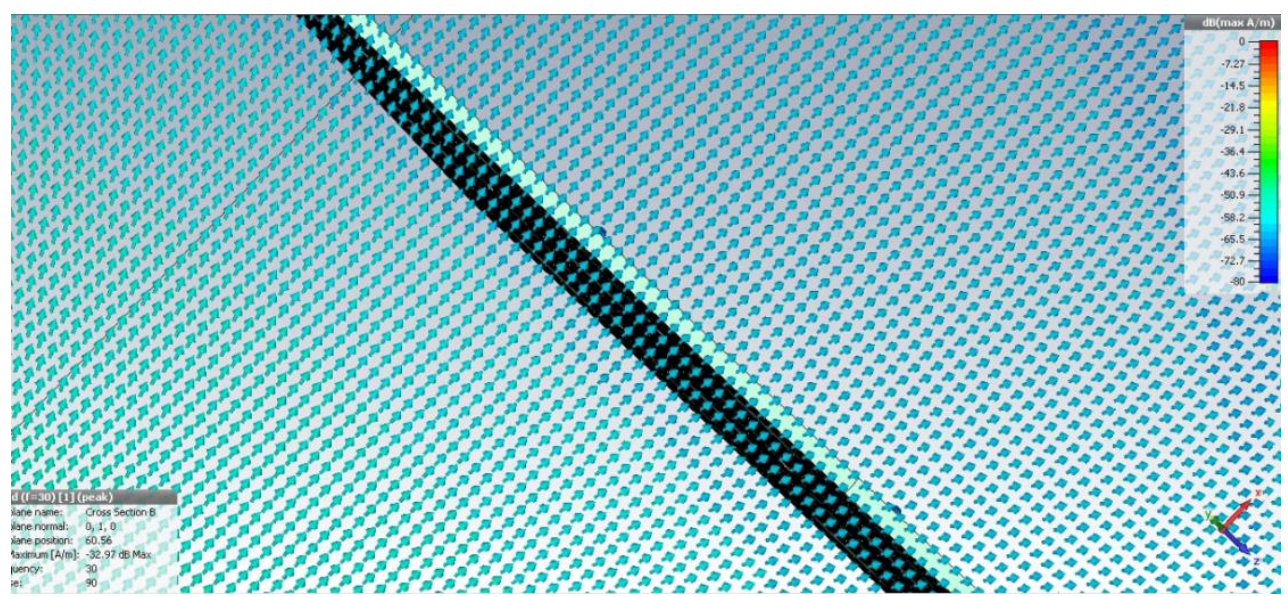

Figure 5.27: The normal component of $H_{z}$ at the surface of the MTM out of the resonance 


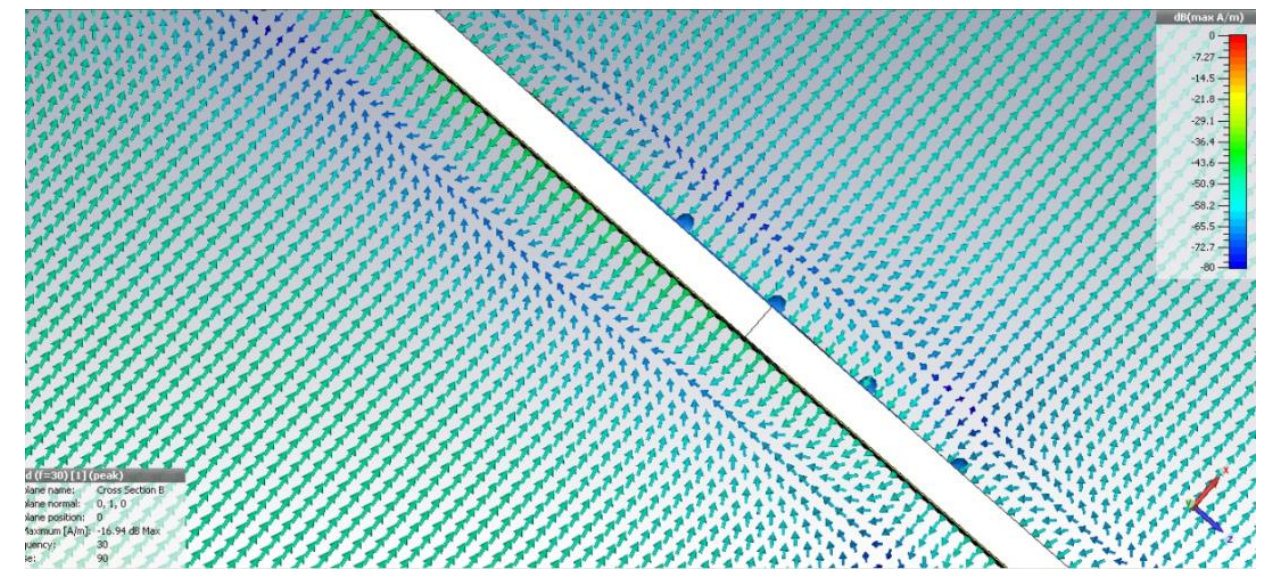

Figure 5.28: The normal component of $H_{z}$ at the surface of the MTM at the resonance

As it can be seen from these field simulations, the normal component of the magnetic field at the surface of the MTM is parallel to the one generated by the source loop out of the resonance (see Figure 5.27) and becomes antiparallel at the resonance (see Figure 5.28).

The boundary conditions of the Maxwell equations determine that normal components of $\boldsymbol{B}$ at the interface are equal [8] [9]:

$$
\begin{gathered}
\boldsymbol{B}_{1, \perp}=\boldsymbol{B}_{2, \perp} \\
\text { if } \boldsymbol{H}_{1, \perp}=-\boldsymbol{H}_{2, \perp}, \quad \text { then } \mu_{1}=-\mu_{2}
\end{gathered}
$$

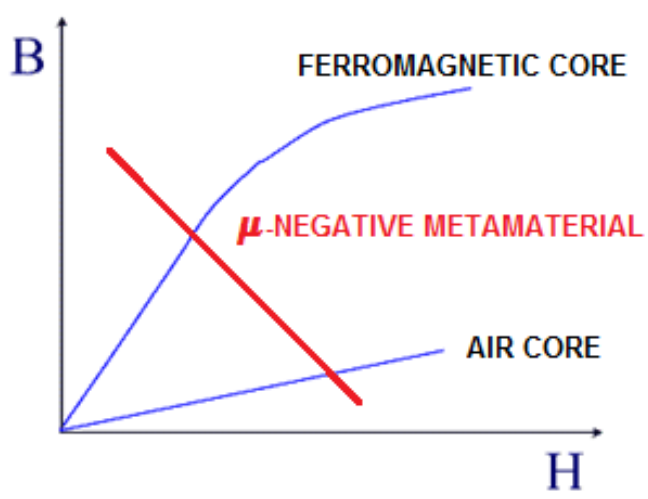

Figure 5.29: Inversion of the magnetization curve derivative

This implies that the magnetization curve of the MTM around the resonance has an inverted derivative as shown in Figure 5.29. 


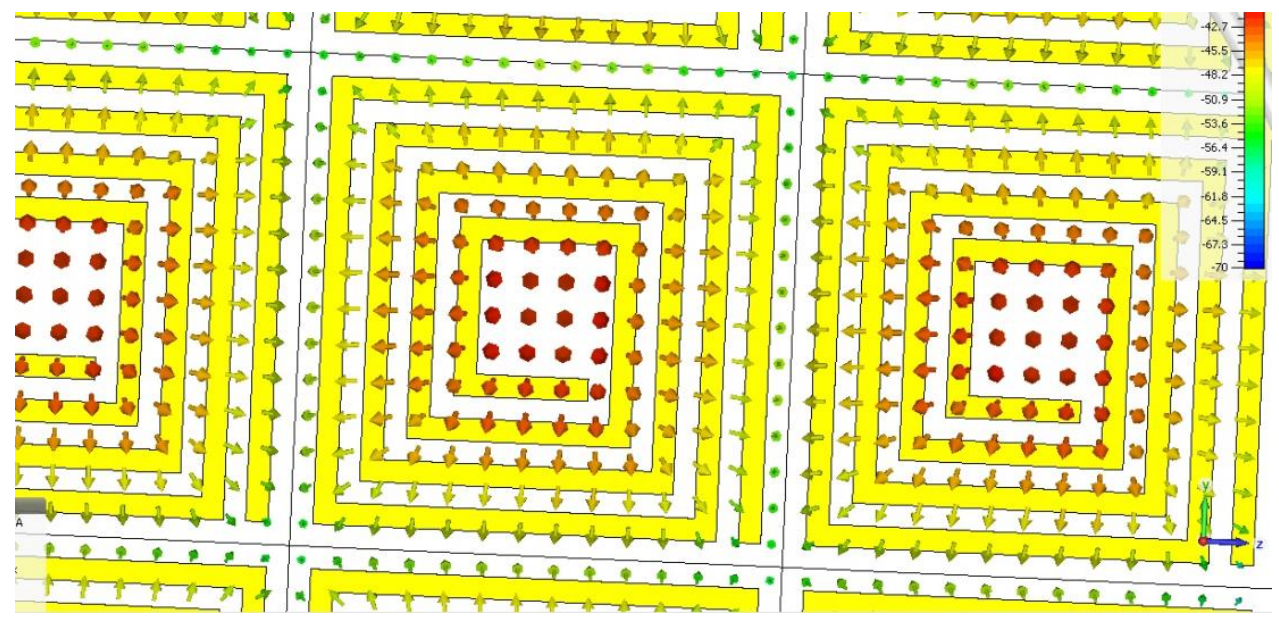

Figure 5.30: The tangential components of $\boldsymbol{H}$ at the surface of the MTM are virtually zero inside the unit-cell area at the resonance

Also, the simulation demonstrates that the tangential components of $\boldsymbol{H}$ at the surface of a $\mu$-negative structure are virtually zero inside the unitcell area (see Figure 5.30), which is the expected behavior of a magnetic conductor $(\mu<0)$.

\section{4 .}

\section{Experimental results for the proposed system}

\subsection{1.}

\section{Experimental protocol}

A Vector Network Analyzer (VNA) is an instrument that measures the network parameters of electrical networks. In the present work, the VNA was used to measure $S$-parameters because reflection and transmission of electrical networks are easy to measure at high frequencies, although there are other network parameter sets, such as Y-parameters, Zparameters and $\mathrm{H}$-parameters, it can measure. VNAs are often used to characterize two-port networks, like amplifiers and filters, but they can be used on networks with an arbitrary number of ports. 


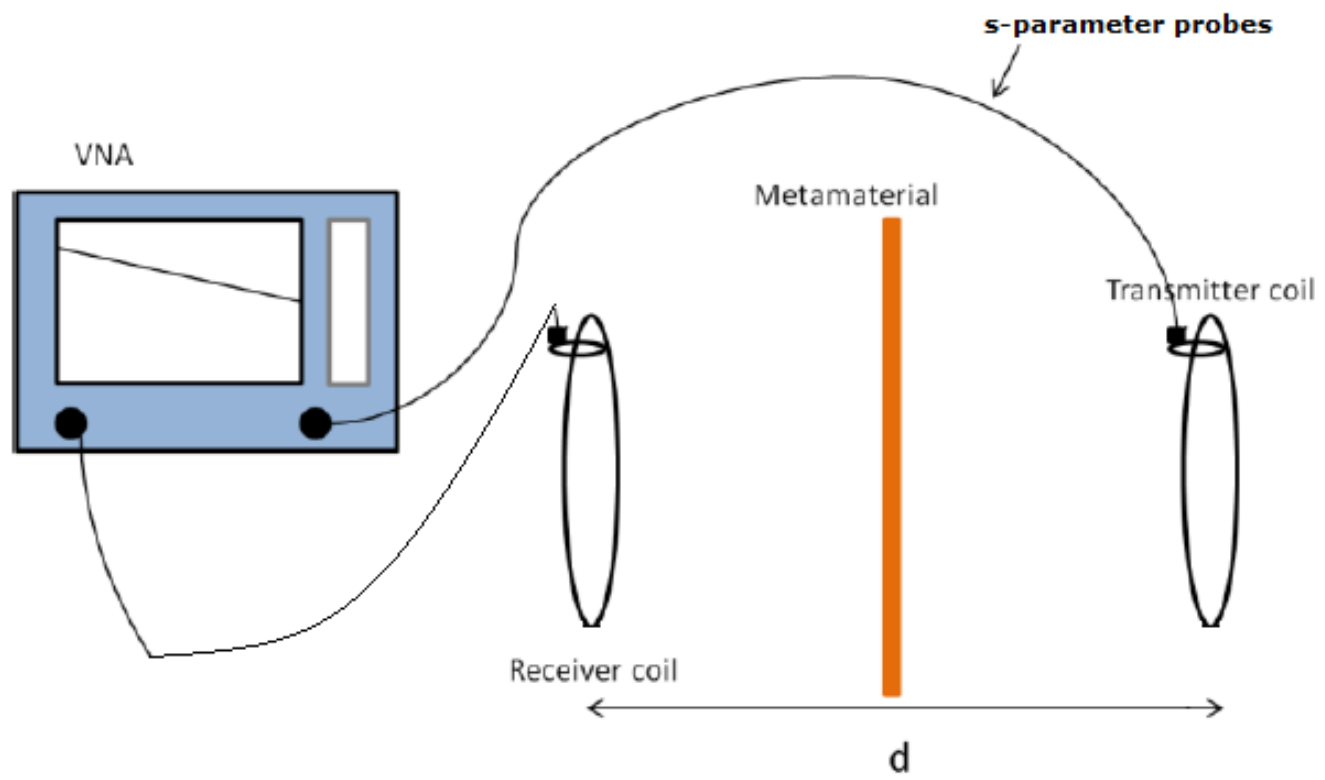

Figure 5.31: Schematic of the setup used to measure the S-parameters of the system

For the S-Parameter measurements, one probe is connected to the transmitting coil and the second one to the receiving coil. The Sparameters are measured and plotted by the VNA as a function of frequency (see Figure 5.31).

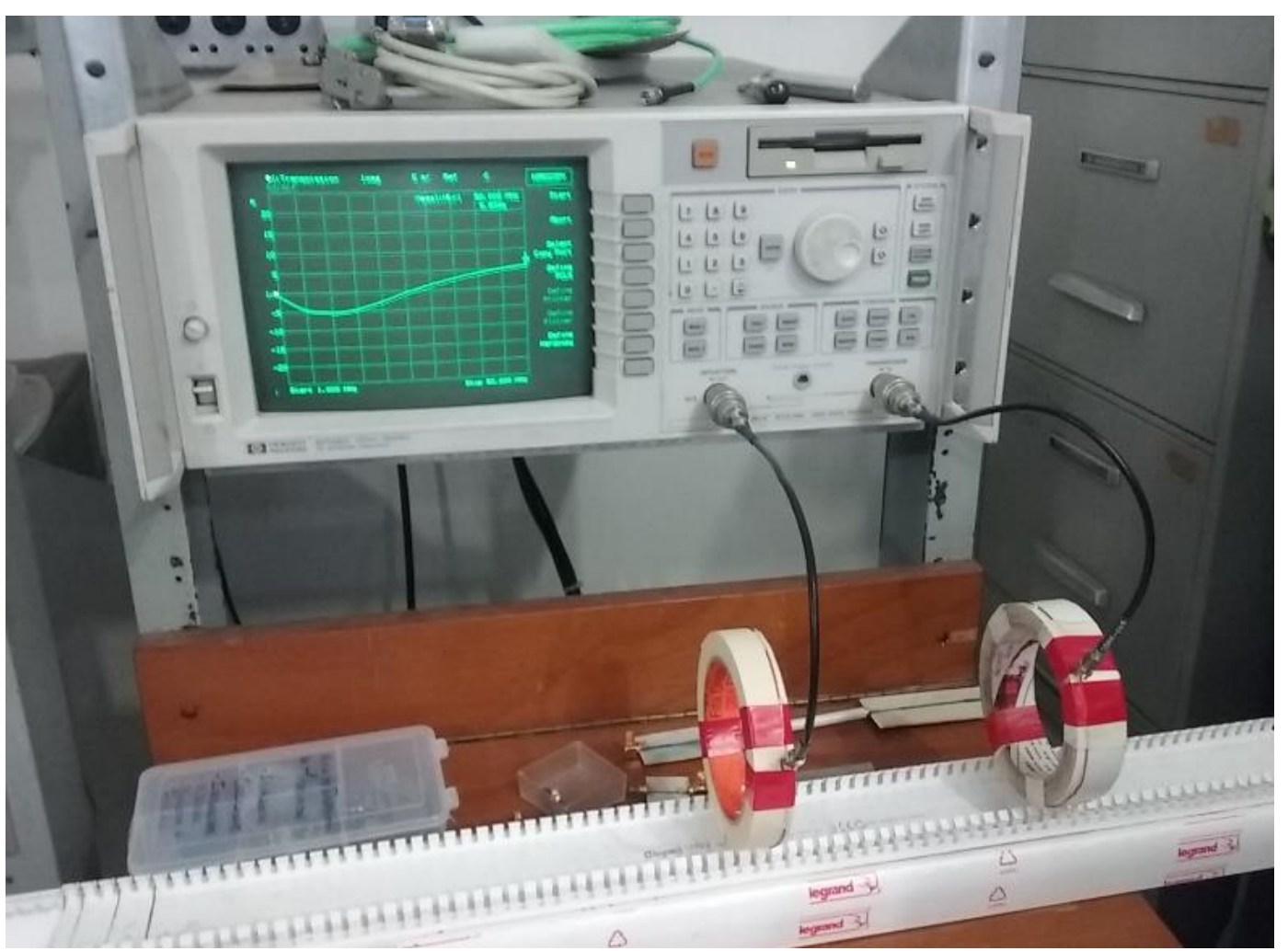

Figure 5.32: Experimental setup without the MTM slab 


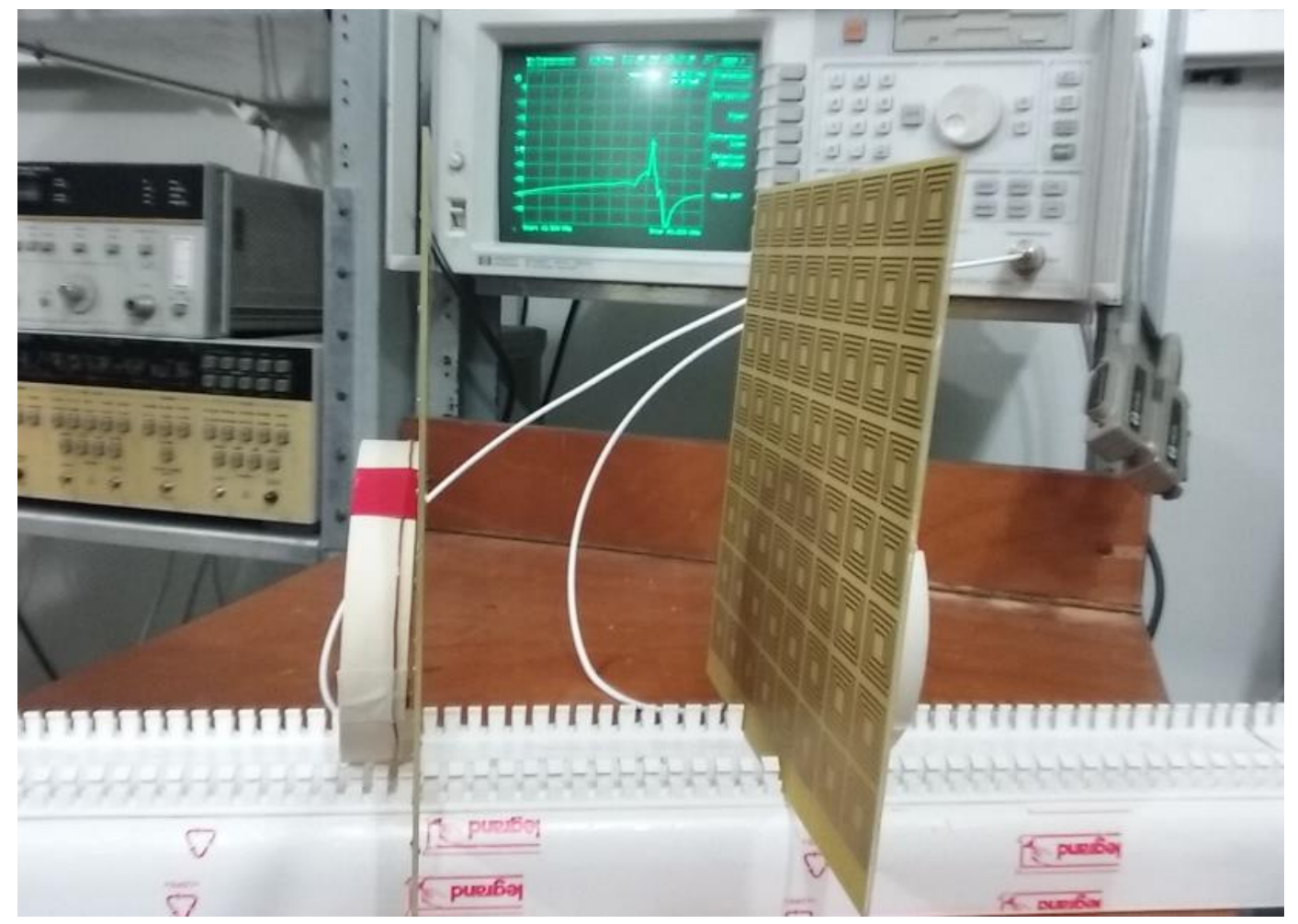

Figure 5.33: Experimental setup with the MTM slab

To evaluate the effect of the MTM over the impedance of the primary circuitry, the port 1 of the VNA (the power supplying one) is connected to the transmitting coil and port 2 is kept opened. The measurement is made at first without (see Figure 5.32) and then with the MTM slab (see Figure 5.33) inside the magnetic link. 
5.4.2.

Results

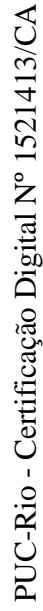

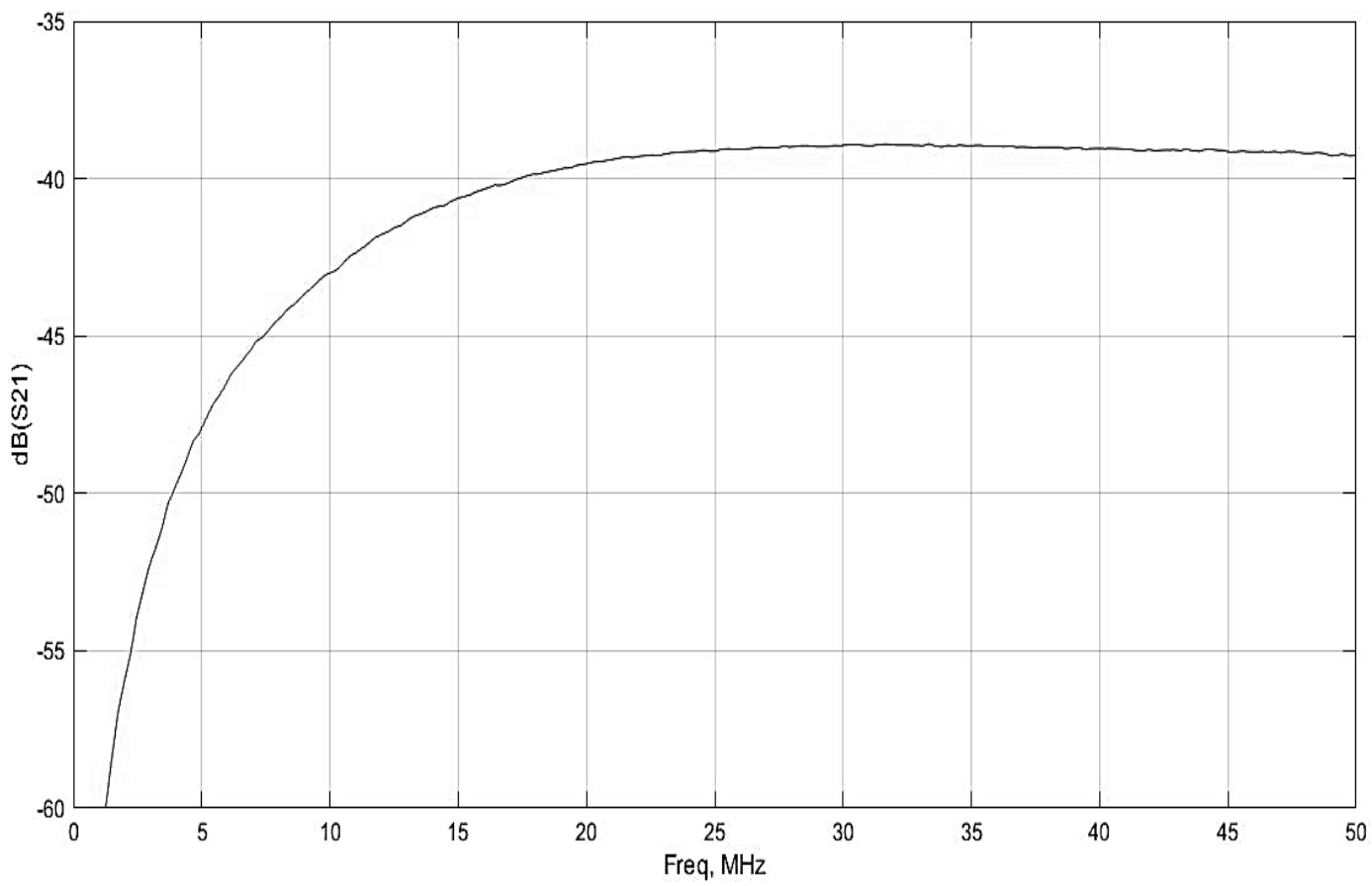

Figure 5.34: Experimental result for the gain $G$ without the MTM slab

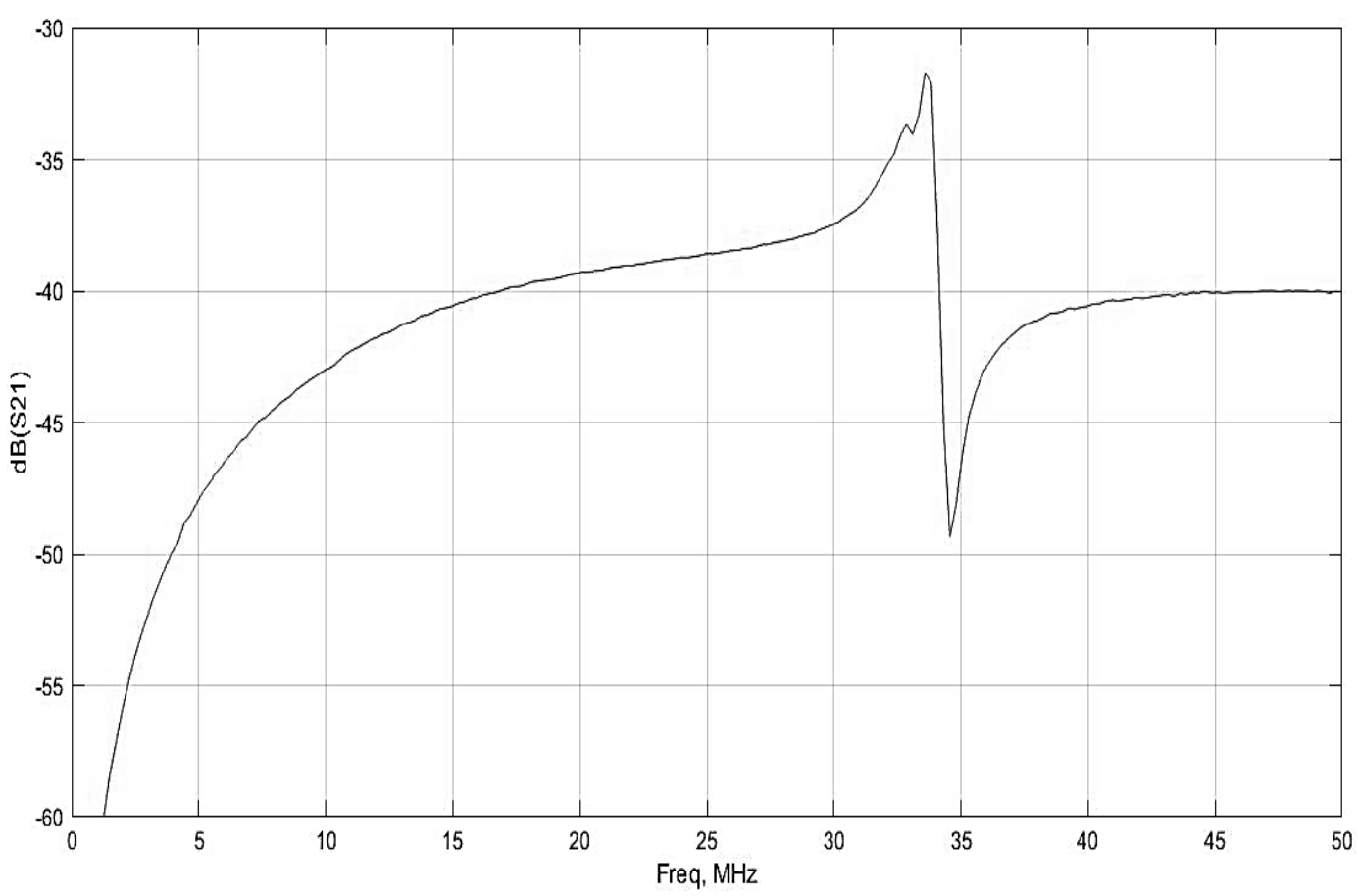

Figure 5.35: Experimental result for the gain $G$ with the MTM slab 
As it can be seen in Figure 5.34 and Figure 5.35, the obtained gain and operating frequency are quite similar to the ones predicted by the analytical model and the numerical simulations. One important aspect of the system is that the relative position of the MTM to the source does not interfere with the gain.

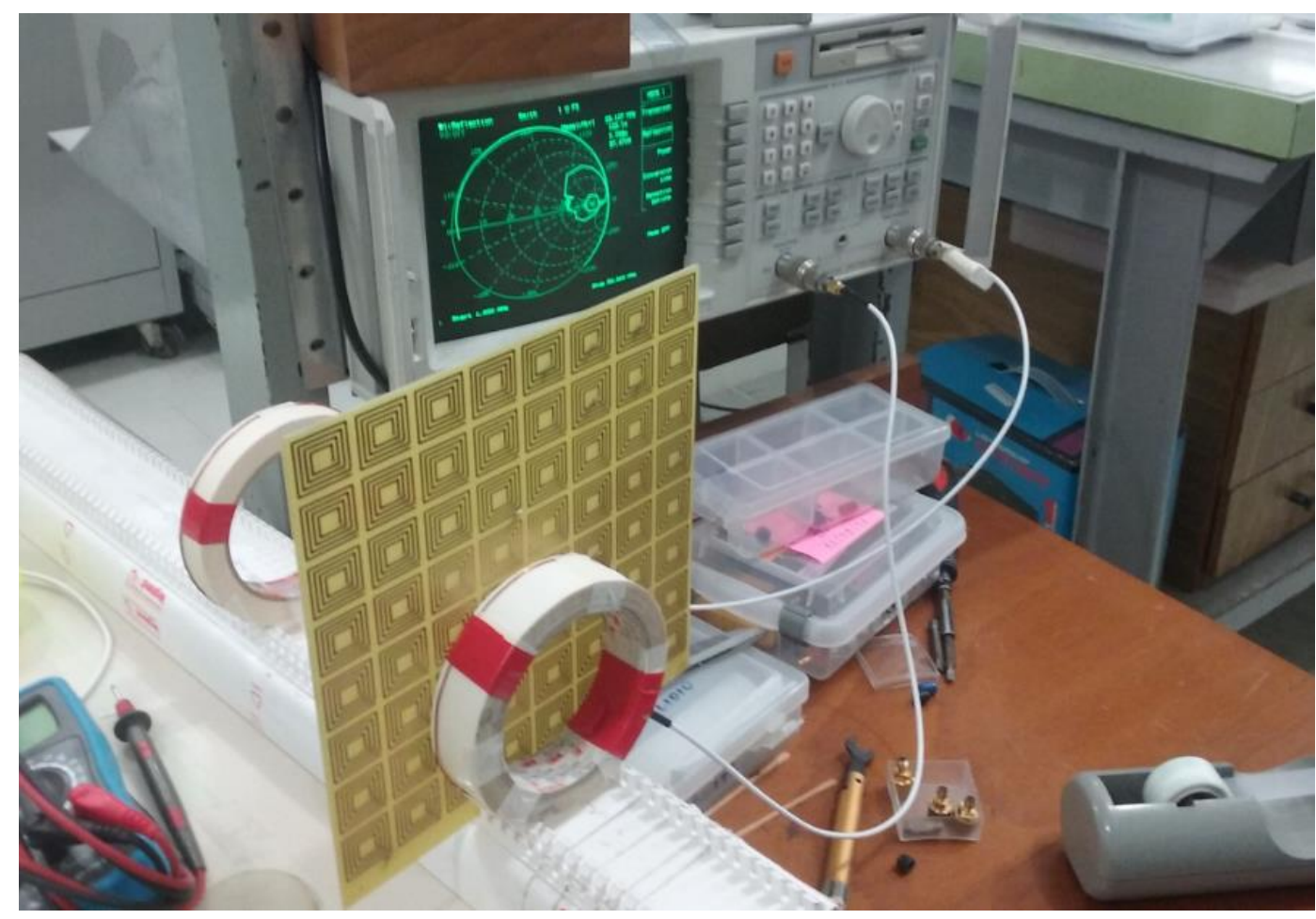

Figure 5.36: The impedance of the source loop is affected if the MTM is too close

If the MTM is close enough to get coupled with the source loop, it directly affects its entrance impedance (see Figure 5.36). In this case, the MTM behaves as a capacitor added to the source at the resonance (see Figure 5.37). 


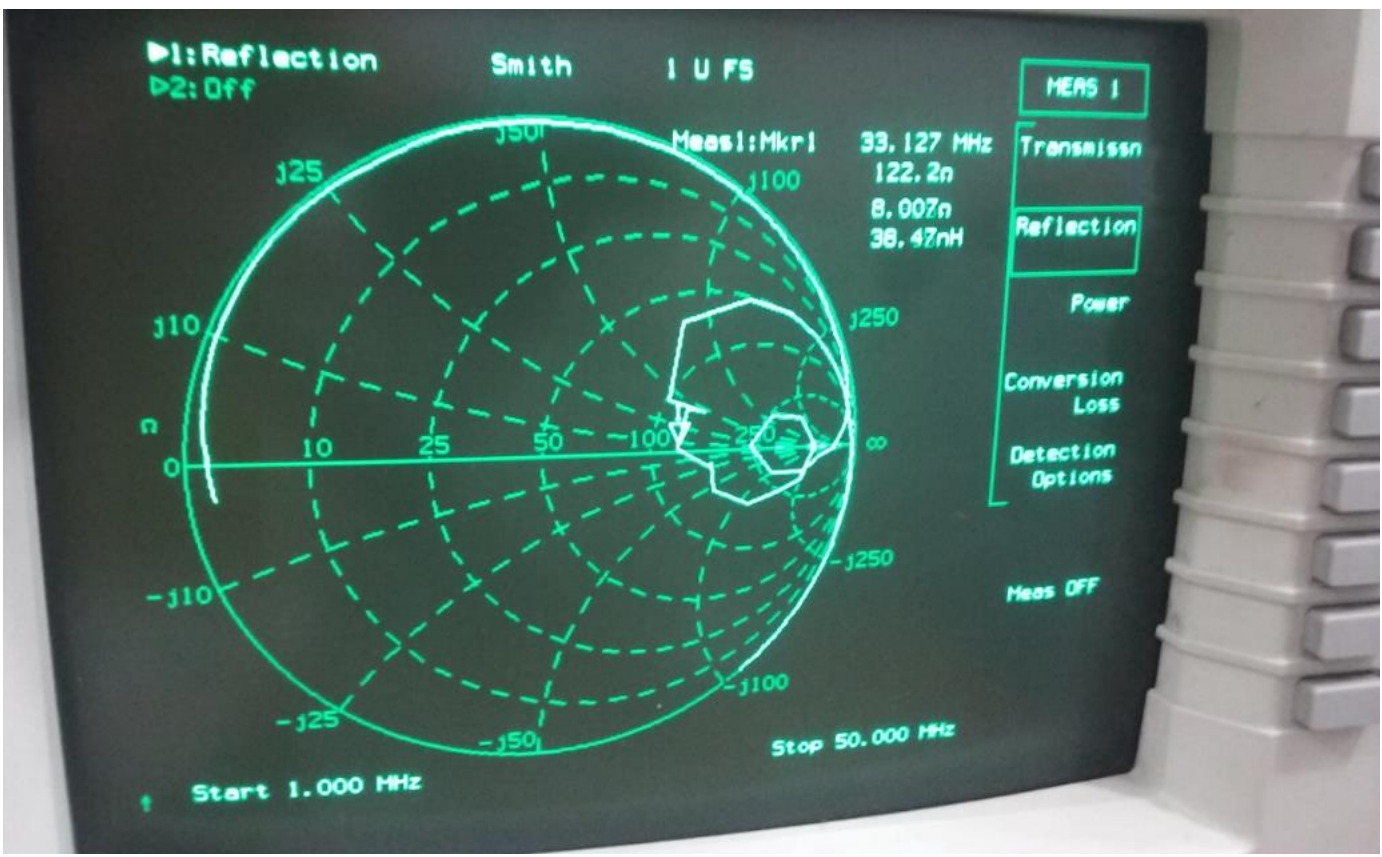

Figure 5.37: The MTM behaves as a capacitor added to the source loop when its separated from it a distance smaller than $a$

To minimize this effect, the MTM must be kept far from the magneticflux source a distance greater than the periodicity $a$ of its lattice. Or, in order to keep compact the transmitting system, the MTM added capacitance should be compensated at the source level.

Just as a final commentary on this aspect, the impedance added by the MTM to the source loop is not related with the gain between the terminals of the virtual line. As it was previously explained, the gain introduced by the MTM is due to the focalization of the magnetic flux and the consequent enhancement of the magnetic potential along the line. The impedance added by the MTM to the source if it is too close would affect the total power transmitted by the tension source connected to the transmitting loop. 


\section{6}

\section{Conclusion}

The main conclusions and results obtained during the realization of this work are resumed in this chapter and its future perspectives are presented.

\section{1.}

\section{Main results}

A time-varying electric or magnetic flux can guide EM modes like physical currents. For this reason, fluxes can be thought as virtual charges and their temporal derivative as virtual currents.

A $\mu$-negative MTM only suffices to focus effectively the magnetic near field.

Above the high-frequency range, the magnetic coupling between two coils in free space, for which the magnetic flux is non-confined, tends to behave similarly to an ideal VMGTL, for which the magnetic flux is perfectly confined.

The MTM-enhanced magnetic coupling is a consequence of the improvement of the impedance matching between the terminals caused by the presence of the MTM in the magnetic link.

The equivalent virtual impedance of the MTM is negative around the resonance frequency and presents a frequency-limited non-Foster behavior, which implies that the MTM is a gain element at its operating frequency. Below the resonance, the MTM is invisible to the virtual line. Above it, it behaves as a positive Foster reactance that stores the energy of the higher-frequency modes.

There is an optimal distance equal to the periodicity $a$ of the MTM lattice that makes the MTM coupling to the virtual line independent of the 
impedance of the source. If the MTM is too close to the transmitting loop, it affects directly the entrance impedance of the source.

\begin{tabular}{|c|c|}
\hline & Gain \\
\hline Analytical & $10 \mathrm{~dB}$ \\
\hline Simulated & $15 \mathrm{~dB}$ \\
\hline Experimental & $9 \mathrm{~dB}$ \\
\hline
\end{tabular}

Table 15: Comparison between the predicted gains and the experimental one

As it can be seen in Table 15, the analytical and experimental results for the MTM gain at the resonance present a greater agreement than the one predicted by the numerical simulation, despite the fact that the curve behavior obtained by simulation is more similar to the one obtained with experiment.

\section{2.}

\section{Contributions}

In the present work it was proposed a generalized TL theory in order to clarify some ambiguities concerning physical and virtual TLs. It was also proposed a discussion concerning the classification of guided and radiated modes in terms of virtual and real potentials.

It was demonstrated that the free-space magnetic coupling above a certain frequency tends to work as an equivalent VMGTL.

It was also demonstrated that the amplification effect does not contradicts the law of conservation of energy since the amplification is due to the exchange of energy between the reactive modes of the near field of the loops. The higher-frequency modes are attenuated by the MTM and the charged MTM becomes a gain medium to the resonance one. During the amplification process, the real part of the Poynting vector is null, so no real power is added to the system.

Finally, it was presented analytical and numerical means to estimate the equivalent virtual impedance represented by $\mu$-negative MTMs and the final gain introduced by it to the system. 


\section{3.}

\section{Future works and perspectives}

Future developments that could be made from this work are:

- Development of probabilistic models to describe the magnetic potential of the free-space VMGTL in order to achieve more accurate results;

- Simulation and realization of the proposed system in a multireceiver scenario;

- Optimization of the MTM lenses via asymmetric resonators and other equivalent techniques to increase the $Q$-factor of their unit cells;

- Optimization of the MTM lenses employing active circuitry in their unit cells;

- Optimization of the geometry of the transmitting and receiving coils to improve the focalization of the magnetic near field;

- Studies on the thermal behavior of the MTM lenses when transmitting higher power levels;

- Studies on the interference of the proposed system on communication systems;

- Studies on the interaction of the proposed IWPT system with living beings and its impact on human and animal health. 


\section{Bibliography}

[1] TESLA, N. My Inventions: The Autobiography of Nikola Tesla. [S.I.]: Start Publishing LLC, 2013.

[2] 6 Companies Pushing Wireless Charging Into The Mainstream, 15 out. 2014. Disponivel em:

<http://www.forbes.com/sites/drewhendricks/2014/10/15/6-

companies-pushing-wireless-charging-into-the-mainstream/>. Acesso em: 12 fev. 2017.

[3] QI Wireless Power Consortium. Disponivel em: <https://www.wirelesspowerconsortium.com/>. Acesso em: 14 fev. 2017.

[4] LIM, Y.; PARK, J. A novel phase control based energy beamforming techniques in non-radiative wireless power transmission. IEEE transactions on power electronics, 2013.

[5] WANG, B. et al. Wireless Power Transfer with Metamaterials. European Conference on Antennas and Propagation. Rome, Italy: [s.n.]. 2011. p. 3905-3908.

[6] WANG, B.; YERAZUNIS, W.; TEO, K. H. Wireless Power Transfer: Metamaterials and Array of Coupled Resonators. Proceedings of the IEEE, 2013.

[7] NISHIMURA, S. I. et al. Enhancing the inductive coupling and efficiency of wireless power transmission system by using metamaterials. 16 $\mathrm{SBMO}$ - Simpósio Brasileiro de Micro-ondas e Optoeletrônica e 11ํ CBMag. Curitiba: MOMAG. 2014.

[8] WENTWORTH, S. M. Fundamentals of Electromagnetics with Engineering Applications. [S.I.]: Wiley, 2006.

[9] HARRINGTON, R. F. Time-Harmonic Electromagnetic Fields. 
[S.I.]: Wiley-IEEE Press , 2001.

[10] KITTEL, C. Introduction to Solid State Physics. [S.I.]: Wiley, 2004.

[11] MONZON, C.; LOSCHIALPO, P.; FORESTER, D. W. Zeropermeability materials: an artifact of losses in left-handed media. IEE Proceedings - Microwaves, Antennas and Propagation, v. 152, n. 6, 2005.

[12] POYNTING, J. H. On the Transfer of Energy in the Electromagnetic Field. Philosophical Transactions of the Royal Society of London, v. 175, p. 343-361, 1884.

[13] BRILLOUIN, L. Wave propagation and group velocity. New York and London: Academic press, 1960.

[14] CAPOLINO, F. Theory and Phenomena of Metamaterials. [S.I.]: CRC Press, 2009.

[15] ENGHETA, N.; ZIOLKOWSKI, R. Metamaterials: physics and engineering explorations. [S.I.]: Wiley \& Sons, Inc, 2006.

[16] DIENER, G. Energy transport in dispersive media and superluminal group velocities. Physical Letters A, 1997. 118-124.

[17] SHIVANAND; WEBB, K. J. Electromagnetic Energy in Negative Index Materials. Antennas and Propagation Society International Symposium (APSURSI). [S.I.]: [s.n.]. 2012.

[18] FARIA, J. A. B.; PIRES, M. P. Theory of Magnetic Transmission Lines. IEEE TRANSACTIONS ON MICROWAVE THEORY AND TECHNIQUES, v. 60, n. 10, 2012.

[19] COLLINS Free Online Dictionary. Disponivel em: $<$ https://www.collinsdictionary.com/dictionary/english/virtual>. Acesso em: 2017 fev. 12.

[20] DARRIGOL, O. Electrodynamics - from Ampère to Einstein. [S.I.]: Oxford University Press, 2000.

[21] FARIA, J. A. B. Poynting Vector Flow Analysis for Contactless. IEEE TRANSACTIONS ON POWER ELECTRONICS, v. 27, n. 10, 
2012.

[22] BANERJEE, S.; DAS, K.; RANA, B. Communication Engineering II. [S.I.]: Vikas Publishing House, 2015.

[23] ATKINS, P. W. The 2nd Law: Energy, Chaos, and Form. [S.I.]: W. H. Freeman, 1994.

[24] VESELAGO, V. G. The electrodynamics of substances with simultaneously negative valus of epslon and mu. Soviet Physics Uspekhi, v. 10, n. 4, 1968.

[25] GRBIC, A.; ELEFTHERIADES, G. V. Overcoming the Diffraction Limit with a Planar Left-Handed Transmission-Line Lens. Physical Review Letters, v. 92, n. 11, 2004.

[26] SMITH, D. R.; PENDRY, J. B.; WILTSHIRE, M. C. K. Metamaterials and negative refractive index. Science, v. 305.

[27] SMITH, D. R.; KROLL, N. Negative Refractive Index in LeftHanded Materials. Physical Review Letters, v. 85, n. 14, p. 29332936, 2000.

[28] SHELBY, R. A.; SMITH, D. R.; SCHULTZ, S. Experimental Verification of a Negative Index of Refraction. Science, v. 292, n. 5514, p. 77-79, 2001.

[29] SMITH, D. R.; PENDRY, J. B. Homogenization of metamaterials by field averaging. Optical Society of America, v. 23, n. 3, p. 391403, 2006.

[30] ANDRYIEUSKI, A.; MALUREANU, R.; LAVRINENKO, A. V. Homogenization of Metamaterials: Parameters Retrieval Methods and Intrinsic Problems. Proceedings of the International Conference on Transparent Optical Networks. [S.I.]: [s.n.]. 2010.

[31] CAPOLINO, F. Applications of Metamaterials. [S.I.]: CRC Press, 2009.

[32] PENDRY, J. B. et al. Magnetism from Conductors and Enhanced Nonlinear Phenomena. IEEE Transactions on Microwave Theory and Techniques, v. 47, n. 11, 1999. 
[33] BILOTTI, F.; TOSCANO, A.; VEGNI, L. Design of Spiral and Multiple Split-Ring Resonatorsfor the Realization of Miniaturized Metamaterial Samples. IEEE Transactions on Antennas and Propagation, v. 55, n. 8, p. 2258-2265, 2007.

[34] WU, J. et al. Wireless Power Transfer with Artificial Magnetic Conductors. IEEE Wireless Power Transfer Conference. [S.I.]: [s.n.]. 2013.

[35] URZHUMOV, Y.; SMITH, D. R. Metamaterial-enhanced coupling between magnetic dipoles for efficient wireless power transfer. Phys. Rev. B, v. 83, n. 205114, 2011.

[36] BOARDMAN, A. D.; MARINOV, K. Electromagnetic energy in a dispersive metamaterial. Physical Review B, v. 73, n. 165110, 2006.

[37] DUARTE, R. M.; FELIC, G. K. Analysis of the Coupling Coefficient in Inductive Energy Transfer Systems. Hindawi Publishing Corporation, n. Active and Passive Electronic Components, 2014.

[38] FOSTER, R. A Reactance Theorem. Bell System Technical Journal, 1926.

[39] ANTENNA Theory. Disponivel em: <http://www.antennatheory.com/antennas/smallLoop.php>. Acesso em: 29 jan. 2016.

[40] MEDHURST, R. G. H. F. Resistance and Self-Capacitance of Single-Layer Solenoids. Wireless Engineer, 1947. p35-43.

[41] EKMEKÇI, E.; TURHAN-SAYAN, G. Investigation of effective permittivity and permeability for a novel v-shaped metamaterial using simulated s-parameters. 5th International Conference on Electrical and Electronics Engineering. [S.I.]: [s.n.]. 2007. 